\title{
Advantages of Unity With SU(4)-Color: Reflections Through Neutrino Oscillations, Baryogenesis and Proton Decay ${ }^{1}$
}

\author{
Jogesh C. Pati ${ }^{23}$ \\ SLAC, Stanford University, Menlo Park, California 94025 USA
}

\begin{abstract}
As a tribute to Abdus Salam, I recall the initiation in 1972-73 of the idea of grand unification based on the view that lepton number is the fourth color. Motivated by aesthetic demands, these attempts led to the suggestion that the existing $S U(2) \times U(1)$ symmetry be extended minimally to the quark-lepton and left-right symmetric nonAbelian gauge structure $G(2,2,4)=S U(2)_{L} \times S U(2)_{R} \times S U(4)$-color. This served to unify members of a family within a single L-R self-conjugate multiplet. It also explained: the quantization of electric charge, the co-existence of quarks and leptons, and that of their three basic forces - weak, electromagnetic, and strong - while providing the appealing possibility that nature is fundamentally left-right symmetric (parityconserving). The minimal extension of the symmetry $G(2,2,4)$ to a simple group is given by the attractive symmetry $S O(10)$ that came a year later. The advantages of the core symmetry $G(2,2,4)$, including those listed above (which are of course retained by $S O(10)$ as well), are noted. These include the introductions of: (i) the right-handed neutrino as a compelling member of each family, (ii) (B-L) as a local symmetry, and (iii) the mass relation $m\left(\nu^{\tau}\right)_{\text {Dirac }}=m_{\text {top }}\left(M_{G U T}\right)$. These three features, all arising due to $S U(4)$-color, as well as the gauge coupling unification scale (identified with the (B-L)- breaking scale), are crucially needed to understand the tiny mass-scales of the neutrino oscillations within the seesaw mechanism, and to implement successfully the mechanism of baryogenesis via leptogenesis. Implications of a well-motivated class of models based on supersymmetric $S O(10)$ or a string-unified $G(2,2,4)$ symmetry in $4 \mathrm{D}$ for (a) gauge coupling unification, (b) fermion masses and mixings, (c) neutrino osillations, (d) baryogenesis via leptogenesis, and last but not least (e) proton decay are presented. Recent works on the latter providing upper limits on proton lifetimes suggest that the potential for discovery of proton decay in the next-generation detectors would be high.
\end{abstract}

\footnotetext{
${ }^{1}$ Presented at the Abdus Salam 90th birthday Memorial Meeting, IAS, Singapore, January 25-28, 2016[1]

${ }^{2}$ E-mail address: pati@slac.stanford.edu.

${ }^{3}$ Work supported in part by the US Department of Energy, contract DE-AC02-76SF00515.
} 


\section{Contents}

1 Introduction $\quad 2$

1.1 Salam in Perspective . . . . . . . . . . . . . . . . . . 2 2

1.2 Aspects of My Collaboration with Salam . . . . . . . . . . . . . . 3

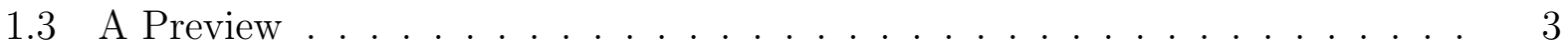

2 Status of Particle Physics in 1972: The Growth of New Ideas 4

2.1 From $S U(2) \times U(1)$ to $G(2,2,4)$ and Beyond . . . . . . . . . . . . 4

2.2 Advantages of the Standard Model . . . . . . . . . . . . . . . . . . 7

2.3 Why Choose The Symmetry $G(2,2,4)$ ? . . . . . . . . . . . . . . . . 8

2.4 The Unwanted Right-Handed Neutrino . . . . . . . . . . . . . . . . . . . . 10

2.5 Left-Right Symmetry in the Fundamental Laws: . . . . . . . . . . . . . . . . 11

2.6 Non-Conservations of B and L: Proton Decay as a Generic Feature . . . . . . 16

2.7 Going Beyond $\mathrm{G}(2,2,4): S O(10)$ and $S U(5) \ldots \ldots . . \ldots$

3 Gauge Coupling Unification 18

3.1 SUSY SO(10) Versus a String-Unified G(2,2,4) Symmetry in 4D . . . . . . 20

4 Breaking $\mathbf{S O}(\mathbf{1 0})$ or $G(2,2,4)$ to the $\mathbf{S M}$

5 Masses and Mixings of Fermions in $\mathbf{S O}(\mathbf{1 0})$ or $G(2,2,4)$

6 Neutrino Masses Shedding Light on Unification and Our Origin

6.1 Neutrinos at the root of our Origin? . . . . . . . . . . . . 39

7 Proton Decay: The Hallmark of Grand Unification 41

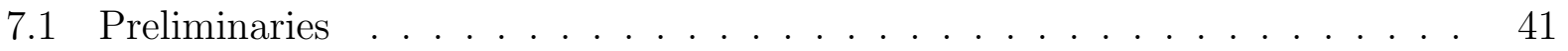

7.2 Constraining Proton Lifetime in SUSY SO(10) with Stabilized Doublet-Triplet Splitting . . . . . . . . . . . . . . . . . . . . 48

7.3 Proton Decay as a Unique Probe to Physics at Ultrashort Distances . . . . . 51

8 Concluding Remarks 


\section{Precis}

By way of paying tribute to Abdus Salam, I first recall the ideas of higher unification that the two of us introduced in 1972-73 to remove certain shortcomings in the status of particle physics prevailing then, and then present their current role in theory as well as experiments. These attempts initiated the $i d e a$ of grand unification and provided the core symmetry-structure $G(2,2,4)=S U(2)_{L} \times S U(2)_{R} \times S U(4)$ - color towards such a unification. Embodied with quark-lepton unification and left-right symmetry, the symmetry $G(2,2,4)$ is uniquely chosen as being the minimal one that permits members of a family to belong to a single multiplet. The minimal extension of $G(2,2,4)$ to a simple group is given by the attractive $\mathrm{SO}(10)$-symmetry that was suggested a year later. The new concepts, and the many advantages introduced by this core symmetry (which are, of course, retained by SO(10) as well) are noted. These include explanations of the observed: (i) (rather weird) electroweak and color quantum numbers of the members of a family; (ii) quantization of electric charge; (iii) electron-proton charge-ratio being -1; (iv) the co-existence of quarks and leptons; (v) likewise that of the three basic forces - the weak, electromagnetic and strong; (vi) the non-trivial cancelation of the triangle anomalies within each family on symmetry grounds; and opening the door for (vii) the appealing concept of parity being an exact symmetry of nature at the fundamental level.

In addition, as a distinguishing feature, both because of SU(4)-color and independently because of $\mathrm{SU}(2)_{\mathrm{R}}$ as well, the symmetry $G(2,2,4)$ introduced, to my knowledge, for the first time in the literature: (viii) a new kind of matter — the right-handed ( $\mathrm{RH}$ ) neutrino $\left(\nu_{R}\right)$ - as a compelling member of each family, and together with it; (ix) (B-L) as a local symmetry. The RH neutrinos - contrary to prejudices held in the 1970's against neutrinos being massive and thereby against the existence of $\nu_{R}$ 's as well - have in fact turned out to be an asset. They are needed to (a) understand naturally the tiny mass-scales observed in neutrino oscillations by combining the seesaw mechanism together with the unification ideas based on the symmetry $\mathrm{SU}(4)$-color, and also (b) to implement the attractive mechanism of baryogenesis via leptogenesis. The quantitative success of the attempts as regards understanding both (a) and (b) are discussed in Sec. 6. These provide a clear support simultaneously for the following three features: (i) the seesaw mechanism, (ii) the SU(4)-color route to higher unification based on a symmetry like $\mathrm{SO}(10)$ or a string-derived $G(2,2,4)$ symmetry in $4 \mathrm{D}$, as opposed to alternative symmetries like $\mathrm{SU}(5)$ or even $[\mathrm{SU}(3)]^{3}$, and (iii) the (B-L) - breaking scale being close to the gauge coupling unification scale $\sim 2 \times 10^{16} \mathrm{GeV}$.

The observed dramatic meeting of the three gauge couplings in the context of low-energy supersymmetry, at a scale $\mathrm{M}_{\mathrm{U}} \sim 2 \times 10^{16} \mathrm{GeV}$, providing strong evidence in favor of the ideas of both grand unification and supersymmetry, is discussed in Sec. 3. The implications of such a meeting in the context of string-unification are briefly discussed. Weighing the possibility of a stringy origin of gauge coupling unification versus the familiar problem of doublettriplet splitting in supersymmetric $\mathrm{SO}(10)$ (or $\mathrm{SU}(5)$ ), I discuss the common advantages as well as relative merits and demerits of an effective $\mathrm{SO}(10)$ versus a string-derived $G(2,2,4)$

symmetry in 4D. Some desirable features of five or six-dimensional orbifold GUT models are noted in here as well,

In Sec. 7, I discuss the hallmark prediction of grand unification, viz. proton decay, which 
is a generic feature of most models of grand unification. I present results of works carried out in collaboration with Babu and Wilczek and most recently with Babu and Tavartkiladze on expectations for decay modes and lifetimes for proton decay, including upper limits for such lifetimes, in the context of a well-motivated class of supersymmetric $\mathrm{SO}(10)$-models. In view of such expectations, I stress the pressing need for having the next-generation large underground detectors — like DUNE and HyperKamiokande — coupled to long-baseline neutrino beams to search simultaneously with high sensitivity for (a) proton decay, (b) neutrino oscillations and (c) supernova neutrinos. It is remarked that the potential for major discoveries through these searches would be high.

Some concluding remarks on the invaluable roles of neutrinos and especially of proton decay in probing physics at the highest energy scales are made in the last section. The remarkable success of a class of supersymmetric grand unification models (discussed here) in explaining a large set of distinct phenomena is summarized. Noticing such a success and yet its limitations in addressing some fundamental issues within its premises, such as an understanding of the origin of the three families and that of the observed dark energy ( cosmological constant), some wishes are expressed on the possible emergence of a suitable grand-unified theory in $4 \mathrm{D}$, together with a resolution of the issues as above in the context of a well-understood quantum theory of of gravity, such as what one might expect from a better understanding of string/M-theory.

\section{Introduction}

\subsection{Salam in Perspective}

Abdus Salam was a rare phenomenon: a great scientist, a humanitarian and a strong promoter of his message that science is the common heritage of all mankind. He will surely be remembered for his many seminal contributions to physics, some of which have proven to be of lasting value. These include his pioneering work on electroweak unification for which he shared the Nobel Prize in physics in 1979 with Sheldon Glashow and Steven Weinberg.

But I believe his most valuable contribution to science and humanity, one that is perhaps unparalleled in the world, is the sacrifice he has made of his time, energy and personal comfort, including his family-life, in promoting the cause of science in the third world. His lifelong efforts in this direction have led to the creation of some outstanding research centres, including especially the International Centre for Theoretical Physics (ICTP) at Trieste, Italy $]_{1}^{1}$ an International Centre for Genetic Engineering and Biotechnology with components in Trieste and Delhi, and an International Centre for Science and High Technology in Trieste. All these centres focus on serving scientists from the third world. Salam dreamed of creating twenty international centres like the ICTP, spread throughout the world, emphasizing different areas of science and technology. Approaching developed as well as developing nations, for funding of such institutions, Salam often used the phrase: "science is not cheap, but expenditures on it will repay tenfold" 2]. Salam's dreams in this regard could not come

\footnotetext{
${ }^{1}$ Now named the Abdus Salam International Centre for Theoretical Physics.
} 
to fruition during his lifetime due to his illness in his later years. Fortunately, they have begun to be realized in part recently through the creation of ICTP partner institutions in Brazil, Mexico, Rwanda and China, thanks to the initiative of the present director of ICTP, Fernando Quevedo, and others.

\subsection{Aspects of My Collaboration with Salam}

My close collaboration with Salam started spontaneously through a tea conversation in the summer of 1972, during my short visit to ICTP, Trieste, and remained strong for over a decade. $2^{2}$ Of this period, I treasure most the memory of many moments which were marked by the struggle and the joy of research that we both shared. While I have mentioned this in my previous writings, 3, 4,5, befitting the present occasion, let me mention again one aspect of Salam's personality. During the ten year period of our collaboration, there have been many letters, faxes, arguments over the phone and in person and even heated exchanges, about tastes and judgements in physics, but always in a good natured spirit. In our discussions, Salam had some favorite phrases. For example, he would sometimes come up with an idea and get excited. If I expressed that I did not like it for such and such reason, he would get impatient and say to me: "My dear sir, what do you want: Blood?" I would sometimes reply by saying: "No Professor Salam, I would like something better". Whether I was right or wrong, he never took it ill. It is this attitude on his part that led to a healthy collaboration and a strong bond between us. Most important for me, by providing strong encouragement from the beginning, yet often arguing, he could bring out the best in a collaborator. For this I will remain grateful to him.

\subsection{A Preview}

As a preview of the topics to be covered in the following sections, I first recall in Sec. 2 the status of particle physics existing in mid-1972, together with its shortcomings, and then present the ideas of higher unification which Salam and I introduced in 1972-73 [6,7,8,9] to remove some of these shortcomings. Current status of these ideas in the context of subsequent developments in theory and experiments are discussed in Secs. 2-6. To begin with, this would include a discussion in Sec. 2 of the chronological evolution of the ideas on unification during 1972-74 starting from: (1) those of the standard model symmetry $G(2,1,3)=S U(2)_{L} \times U(1)_{Y} \times S U(3)$-color; to (2) the minimal quark-lepton and left-right symmetric non-Abelian symmetry $\mathrm{G}(2,2,4)=\mathrm{SU}(2)_{L} \times \mathrm{SU}(2)_{R} \times \mathrm{SU}(4)$-color [8,9] that brought a host of attractive features by removing some of the major shortcomings of the standard model and introducing certain new ingredients, like the right-handed $(\mathrm{RH})$ neutrinos $\left(\nu_{\mathrm{R}}\right.$ 's), that turned out to be an asset (See Secs. 2, 5 and 6); to (3) the smallest left-right asymmetric simple group $\mathrm{SU}(5)$ [10], containing the standard model symmetry $\mathrm{G}(2,1,3)$, that

\footnotetext{
${ }^{2} \mathrm{~A}$ brief account of how our collaboration started in May 1972 leading to the ideas of higher unification and to the origin of the notion of "Lepton Number as the Fourth Color", in this context, is given in my article in the Proceedings of the Salamfestschrift [3] which was held at ICTP in 1993 (that is probably the last scientific meeting that Salam attended), and a shorter version is given in the articles written in his honor after he passed away 4,5].The first two sections of this talk are based in part on these three articles.
} 
had the virtue of demonstrating explicitly the idea of grand unification; to (4) the minimal extension of $\mathrm{G}(2,2,4)$ to the attractive simple group $\mathrm{SO}(10)$ [1] that possesses all the benefits of $\mathrm{G}(2,2,4)$, and in addition offers gauge coupling unification. $\mathrm{SO}(10)$ even retains the left-right self-conjugate 16-component family-structure of $\mathrm{G}(2,2,4)$, as opposed to the 15 component family, composed of $(\overline{5}+10)$ of $\mathrm{SU}(5)$. The single extra member in the 16-plet of $\mathrm{G}(2,2,4)$ or $\mathrm{SO}(10)$ is the $\mathrm{RH}$ neutrino.

The special advantages of the $\mathrm{SU}(4)$-color route to higher unification offered by the symmetry $\mathrm{G}(2,2,4)$, and therefore $\mathrm{SO}(10)$ as well, are noted in Secs. 2, 4, 5 and 6. These include an understanding of the tiny mass-scales observed in neutrino oscillations [12,13], in the context of the seesaw mechanism[14], as well as implementing the promising mechanism of baryogenesis via leptogenis [15,16]. These desirable features are not available, however, within the alternative routes of $\mathrm{SU}(5)$ or even $[\mathrm{SU}(3)]^{3}[17]$.

Implications of the precision measurements of the three gauge couplings at LEP, revealing their unification [18,19] in the context of low-energy supersymmetry [20,21], are discussed in Sec. 3. The changes in theoretical perspective pertaining to gauge coupling unification and proton decay 22] brought about by the ideas of supersymmetry and superstrings [23] are discussed briefly in here, as well.

In Sec. 7, I discuss works carried out in collaboration with Babu and Wilczek [24,25] and more recently with Babu and Tavartkiladze 26,27] on expectations for decay modes and lifetimes of proton decay, including upper limits, in the context of a well-motivated class of supersymmetric $\mathrm{SO}(10)$ models. It is stressed that these expectations are within a striking range of the next-generation detectors being planned at DUNE and HyperKamiokande. Some concluding remarks are made in Sec. 8.

\section{Status of Particle Physics in 1972: The Growth of New Ideas}

\subsection{From $S U(2) \times U(1)$ to $G(2,2,4)$ and Beyond}

As noted above, my collaboration with Salam started during my two-months visit to ICTP, Trieste in the summer of 1972. To put the growth of ideas during that summer in perspective, I will first provide a historical background of the status of particle physics existing in May 1972 and then provide motivations for the idea of higher unification, which developed over the next two years. This was a time when the electroweak $\mathrm{SU}(2) \times \mathrm{U}(1)$ model 28] based on the Higgs mechanism for symmetry breaking [29] existed. And the renormalizability of such theories had been proven [30] creating much excitement in the field.

But there was no clear idea of the origin of the fundamental strong interaction. The latter was thought to be generated, for example, by the vector bosons $\left(\rho, \omega, \mathrm{K}^{*}\right.$ and $\left.\phi\right)$ along the lines suggested ${ }^{3}$ by Sakurai, [31] inspired by the beautiful Yang-Mills idea [32], or even by the spin-o mesons $(\pi, \mathrm{K}, \eta, \eta, \sigma)$ assumed to be elementary, or a neutral $\mathrm{U}(1)$ vector gluon

\footnotetext{
${ }^{3}$ Although the idea of generating strong interactions by the gauge principle is attractive, it might have been argued that the flavor-SU(3) gauge symmetry is not a suitable choice to generate the fundamental
} 
coupled universally to all the quarks 33$]$.

By this time, based on the need to satisfy Pauli principle for the baryons treated as three-quark composites, the idea of SU(3)-color as a global symmetry had been introduced implicitly with quarks satisfying parastatistics of rank 3 in [34] and explicitly though quarks obeying familiar Fermi-Dirac statistics in 35. In this context, the suggestion of generating a "superstrong" force by gauging the SU(3)-color symmetry had also been made by Han and Nambu as early as in 1965 [35], though in a variant form compared to its present usage (see remarks later). But the existence of the $\mathrm{SU}(3)$-color degree of freedom even as a global symmetry was not commonly accepted in 1972 because many thought that this would require an undue proliferation of elementary entities. And, of course, asymptotic freedom had not been discovered yet. Thus the standard model including the SU(3)-color symmetry had not been born,

In the context of such a background, inspired by 't Hooft's proof of renormalizability of spontaneously broken gauge theories, there was a number of papers appearing almost daily at the ICTP preprint library which tried to build variants of the $\mathrm{SU}(2) \times \mathrm{U}(1)$ model. For example, there were even attempts [36] to get rid of the weak neutral current weak interactions because experiments at that time (May 1972) hinted at their absence.

As I was trying to catch up with these papers, it appeared to me that the heart of the matter laid not in trying to find variants of the $\mathrm{SU}(2) \times \mathrm{U}(1)$ model, but in removing its major shortcomings, first in its gauge sector. These included: (i) in particular the arbitrary choice of the five scattered multiplets for each family consisting of quarks and leptons with rather weird assignment of their quantum numbers including the weak hypercharge which were put in by hand without a guiding principle; (ii) the lack of a reason based on symmetry arguments for the co-existence of quarks and leptons, and likewise (iii) that of the three forces-weak, electromagnetic and strong; and (iv) the absence of a compelling reason for the quantization of the electric charge and that for the observed charge-relation: $Q_{\text {electron }}=-Q_{\text {proton }}$ (v) In addition, I was bothered by the disparity with which the $\mathrm{SU}(2) \times \mathrm{U}(1)$ model treated the left and the right chiral fermions (see Eq. (1)). This amounted to putting in non-conservation of parity by hand. I thought (in Pauli's words) that God can't be weakly left-handed and at a deeper level the underlying theory ought to treat left and right on par, conserving parity.

I mentioned these concerns of mine, based on aesthetic grounds, about the $\mathrm{SU}(2) \times \mathrm{U}(1)$ model to Salam at a tea-gathering at ICTP[3]. I also expressed that in order to remove these shortcomings one would need to put quarks and leptons in one common multiplet of a higher symmetry group (so that one may understand the co-existence of quarks and leptons and explain why $\mathrm{Q}_{\mathrm{e}^{-}}=-\mathrm{Q}_{\mathrm{p}}$ ) and gauge such a symmetry group to generate simultaneously the weak, electromagnetic and strong interactions in a unified manner.

Now, the idea of putting quarks and leptons in the same multiplet was rather unconventional at that time. Rather than expressing any reservation about it, as some others did, Salam responded immediately by saying "That seems like an excellent idea! Let us develop

strong interaction because weak interaction (viewed perturbatively) was "known" to use part of the same symmetry, as in the $\mathrm{SU}(2) \times \mathrm{U}(1)$ model. This would suggest that $\left(\rho, \omega, \mathrm{K}^{*}\right)$ are not fundamental gauge bosons. 
it together". It is this sort of spontaneous appreciation and encouragement from Salam that helped to enrich our collaboration at every step. Thus started our collaboration from that tea-conversation.

Searching for a higher symmetry to incorporate the features noted above, it became clear within about two weeks ${ }^{2}$ that quarks and leptons can be united in an elegant manner by assuming that quarks do in fact possess the SU(3)-color degree of freedom . $^{4}$ obeying the familiar Fermi-Dirac statistics 35] rather than parastatistics [34], like the electrons do, and extending $\mathrm{SU}(3)$-color to the gauge symmetry $\mathrm{SU}(4)$-color that treats lepton number as the fourth color. Within this picture, the neutrino and the electron emerged as just the up and down "quarks" of lepton color.

With SU(4)-color, the whole spectrum of quarks and leptons (then consisting of only two families) fitted beautifully into a $4 \times 4$ structure of a global symmetry group $\mathrm{SU}(4)^{\text {flavor }} \times$ $\mathrm{SU}(4)^{\text {color }}$ operating on four flavors $(\mathrm{u}, \mathrm{d}, \mathrm{c}, \mathrm{s})$ and four colors $(\mathrm{r}, \mathrm{y}, \mathrm{b}, \mathrm{l}) \mathrm{5}^{5}$ Such a structure accounted naturally for the vanishing of the sum of quark and lepton charges and that of the combination $\left(\mathrm{Q}_{\mathrm{e}^{-}}+\mathrm{Q}_{\mathrm{p}}\right)$, as desired. The spontaneous breaking of $\mathrm{SU}(4)$-color at high energies to $\mathrm{SU}(3)^{\mathrm{c}} \times \mathrm{U}(1)_{\mathrm{B}-\mathrm{L}}$ was then suggested to explain the observed distinction between quarks and leptons at low energies, as regards their response to strong interactions; such a distinction must then disappear at sufficiently high energies.

Uniting quarks and leptons by the SU(4)-color gauge symmetry thus naturally implied the idea that the fundamental strong interaction of quarks arises entirely through the octet of gluons generated by its subgroup of the SU(3)-color gauge symmetry, which is exact in the lagrangian [37,38,39].

In the course of our attempt at a higher unification [6,7], it thus followed that the effective gauge symmetry describing electroweak and strong interactions at low energies (below a TeV) must minimally be given by the combined gauge symmetry ${ }^{6} \mathrm{G}(2,1,3)=\mathrm{SU}(2)_{\mathrm{L}} \times \mathrm{U}(1)_{\mathrm{Y}} \times$ $\mathrm{SU}(3)^{\mathrm{c}}$. This became known eventually as the standard model symmetry (SM). It, of course, contains the electroweak symmetry $\mathrm{SU}(2)_{\mathrm{L}} \times \mathrm{U}(1)_{\mathrm{Y}}[28]$.

\footnotetext{
${ }^{4}$ For reasons alluded to in footnote 3 , one could argue that the $\mathrm{SU}(3)$-color degree of freedom of quarks in the explicit sense 35] is essential not only to achieve a higher unification but just to realize a pure gauge-origin of the three forces-weak, electromagnetic and strong (see below). I thank O.W. Greenberg for a discussion on the need of Fermi-Dirac rather than parastatistics especially in the context of grand unification.

${ }^{5}$ Effectively such a $4 \times 4$-structure is to be viewed as a merger of two families each being a $2 \times 4$. In reality, one must of course gauge either the chiral $\mathrm{SU}(4)_{\mathrm{L}}^{\mathrm{f}} \times \mathrm{SU}(4)_{\mathrm{R}}^{\mathrm{f}}$ (by assuming mirror fermions to avoid anomalies), or a suitable anomaly-free subgroup of it. This is what we did (see below). But for the purposes of classification and assignment of electric charge, which is vectorial, it sufficed to use the non-Abelian vectorial $\mathrm{SU}(4)^{\mathrm{f}} \times \mathrm{SU}(4)^{\mathrm{c}}$.

${ }^{6}$ I should comment here on a common impression that exists in the literature, including especially popular writings, as regards the origin of the standard model versus that of the idea of grand unification. It is often stated that the successes of the standard model naturally suggested that it be extended to include grand unification. Historically, the story is, however, quite different. In the beginning of May 1972, neither the standard model in its entirity, including SU(3)-color, nor any of its empirical successes, such as the discovery of weak neutral current interactions, existed. As indicated in the text, the combined gauge symmetry of the standard model emerged 6/7] at this juncture simultaneously with the attempts at higher unification, based (in our case) on SU(4)-color. See further remarks in Ref. [40].
} 
It is instructive to note the family-multiplet structure with respect to the SM symmetry. The 15 two-component members of the electron family belong to five disconnected multiplets under the symmetry $\mathrm{G}(2,1,3)$ as shown below:

$$
\left(\begin{array}{lll}
u_{r} & u_{y} & u_{b} \\
d_{r} & d_{y} & d_{b}
\end{array}\right)_{L}^{\frac{1}{3}} ;\left(\begin{array}{lll}
u_{r} & u_{y} & u_{b}
\end{array}\right)_{R}^{\frac{4}{3}} ;\left(\begin{array}{lll}
d_{r} & d_{y} & d_{b}
\end{array}\right)_{R}^{-\frac{2}{3}} ;\left(\begin{array}{c}
\nu_{e} \\
e^{-}
\end{array}\right)_{L}^{-1} ;\left(e^{-}\right)_{R}^{-2} .
$$

Likewise for the muon and the tau families. Here the superscripts denote the respective weak hypercharges $Y_{W}$ (where $Q_{e m}=I_{3 L}+Y_{W} / 2$ ), which are chosen by hand simply to fit the "observed" electric charges. The subscripts L and R denote the chiralities of the respective fields. The symmetry SU(3)-color acts horizontally treating quarks of three different colors of either chirality in each row as a triplet, while $\mathrm{SU}(2)_{\mathrm{L}}$ acts vertically on each column treating all LH fields as doublets, but all RH ones only as singlets. Note the sharp distinction between the ways the SM treats the left and the right chiral fermions. This is of course needed to conform with observations (at presently available energies). I will discuss shortly how these five disconnected multiplets become parts of a single multiplet under certain higher unification symmetries, which would also treat the left and the right symmetrically.

We wrote up this aspect of our thinking in a short draft, which we submitted to J.D. Bjorken for presentation at the 1972 Batavia conference [6], and then in a paper which appeared in [7]. In here, we suggested the concept of quark-lepton unification through $\mathrm{SU}(4)$-color. In addition, unknown to many, we also initiated in the same paper ( in the third para) the idea of a gauge-unification of the three forces in terms of a single coupling constant, without exhibiting explicitly a symmetry to implement this idea. We conjectured 7 that the differing renormalization effects on the three gauge couplings following spontaneous breaking of the unifying symmetry, may cause the observed differences between these three couplings at low energies. Fortunately, this conjecture (hope) was borne out precisely by the discovery of asymptotic freedom about four months later 41]. I will return to a discussion of the success of the idea of gauge coupling unification in Sec. 3.

\subsection{Advantages of the Standard Model}

Before continuing on the idea of higher unification, certain advantages of the standard model, viewed as an effective low-energy theory, are worthnoting. They include the following:

(i) The triangle anomalies [43] coming from the quarks for this gauge-structure, with the $4 \times 4$-spectrum of quarks and leptons mentioned above, are found to cancel beautifully against those coming from the leptons 44] provided the quarks possess three colors. Such a cancelation, which is crucial to the renormalizability of the theory, thus provided a strong independent evidence in favor of the SU(3)-color degree of freedom of the quarks. The cancelation, which is non-trivial, can not however be an accident. As we will see, a deeper reason for the automatic cancelation of the anomalies for each family would in fact arise on symmetry grounds within certain higher unification symmetries, including some based on SU(4)-color.

\footnotetext{
${ }^{7}$ The fulfillment of this conjecture is, of course, a prerequisite for the idea of grand unification to work. See remarks in Sec. 1 of Ref. [7.
} 
(ii) With the presence of four flavors $(\mathrm{u}, \mathrm{d}, \mathrm{c}, \mathrm{s})$, the standard model naturally incorporates the Glashow-Iliopoulos-Maiani (GIM) protection mechanism[45] in the presence of cabibbo mixing [46] so as to avoid excessive flavor-changing neutral current processes including $\mathrm{K}^{\circ}-\overline{\mathrm{K}}^{\circ}$ mixing (see below for results including radiactive corrections). Such a protection is essentially unaffected when the standard model is extended to incorporate the third family in the context of the Cabibbo-Kobayashi-Masakawa (CKM) mixing, including one CP-violating phase[47].

(iii) With strong interactions generated by $\mathrm{SU}(3)$-color and the electroweak interactions by the commuting $\mathrm{SU}(2)_{\mathrm{L}} \times \mathrm{U}(1)_{\mathrm{Y}^{-}}$symmetry, it was shown 48, that, despite radiative corrections, "violations of parity and of stangeness by one unit are of order $\mathrm{G}_{\mathrm{F}} \mathrm{m}^{2}$ (rather than of order $\alpha$ ) and the $|\Delta S|=2$ transitions $\left(\mathrm{K}^{\circ}-\overline{\mathrm{K}}^{\circ}\right)$ are of order $\left(\mathrm{G}_{\mathrm{F}} \mathrm{m}^{2}\right)^{2}$ as desired, where $\mathrm{m}$ is a typical hadronic mass.

(iv) Importantly, with strong interactions generated entirely by the non-Abelian SU(3)color gauge force, the short-distance processes involving the hadrons are governed by the property of asymptotic freedom[41]. This served to explain the Bjorken scaling[49] in deep inelastic electron-nucleon scattering observed at SLAC, providing justification for the approximate validity of the parton model[50], and subsequently explaining remarkably well a host of short-distance processes including the small logarithmic deviations from scaling in them. In turn, this gave a strong boost to the purely $\mathrm{SU}(3)$-color gauge-origin of the strong force. This defined the theory of quantum chromodynamics (QCD), which is an integral part of the standard model. In addition, the perturbative growth of the QCD coupling at long distances, together with the infrared divergences of the non-Abelian QCD, made the idea of confinement of quarks and massless gluons at least plausible[51], which has been put on a firmer footing by QCD-lattice calculations [52]. In short, QCD with its non-Abelian self-interactions of gluons served to account simultaneously for the two rather mysterious properties of quarks and gluons-i.e. asymptotic freedom at short distances, and yet confinement at long distances.

(v) Last but not least, the standard model provides a renormalizable self-consistent quantum field theory of the three basic forces with enormous predictive power involving a rich variety of phenomena, well beyond those of QED. And, its predictions are brilliantly successful. Even a brief mention of the empirical successes of the standard model, both in its electroweak and the QCD sectors, culminating with the discovery of the Higgs boson in 2012, will take me outside the theme of my talk. At the same time, reflecting on the latter, this discovery seems to reaffirm the view that nature, peculiarly enough, utilizes renormalizability as one of the principles in formulating her laws. In other words, she cares that the laws should possess not only beauty but also reliability in the associated predictions!. Let me then return to the growth of ideas on higher unification.

\subsection{Why Choose The Symmetry $G(2,2,4)$ ?}

In searching for a desirable symmetry to meet certain aesthetic demands there appeared to be two different ways which lead to the same answer as regards the choice of such a symmetry. First, if one asks the question: which is the minimal gauge symmetry that contains quarklepton unification through $\mathrm{SU}(4)$-color, together with the electroweak symmetry $\mathrm{SU}(2)_{\mathrm{L}} \times$ 
$\mathrm{U}(1)_{\mathrm{Y}}$, and simultaneously provides a rationale for the quantization of electric charge, the answer is clear and simple. One must minimally gauge the quark-lepton and left-right symmetric gauge structure [8,9]:

$$
G(2,2,4) \equiv S U(2)_{L} \times S U(2)_{R} \times S U(4)^{c} .
$$

Note the need for the non-Abelian left-right symmetric flavor gauge-structure $\mathrm{SU}(2)_{\mathrm{L}} \times$ $\mathrm{SU}(2)_{\mathrm{R}}$ (rather than $\mathrm{SU}(2)_{\mathrm{L}} \times \mathrm{I}_{3 \mathrm{R}}$ ), accompanying $\mathrm{SU}(4)$-color, that arises due to the requirement of quantization of electric charge, together with that of minimality. Here $\mathrm{SU}(2)_{\mathrm{L}}$ and $\mathrm{SU}(2)_{\mathrm{R}}$ are the exact left-right analogs of each other. While $\mathrm{SU}(2)_{\mathrm{L}}$ groups the LH fermions of a family into doublets (see Eq. (1)), $\mathrm{SU}(2)_{\mathrm{R}}$ does the same for the corresponding $\mathrm{RH}$ fermions, thereby providing the basis for left-right symmetry in the gauge interactions. The deeper implications of left-right symmetry will be noted shortly following the presentation of the family-multiplet structure in Eq. (3), with respect to the symmetry $\mathrm{G}(2,2,4)$.

Before moving on, it is interesting to note that the need for choosing $\mathrm{G}(2,2,4)$ as the minimal symmetry arises by starting from a completely different angle. Without assuming $S U(4)$-color or the L-R symmetric gauge structure to begin with, if one just asks the question: which is the minimal gauge symmetry that would group the five disconnected multiplets of the SM belonging to a family (see Eq. (1)) into a single multiplet, then the answer is simple and unique. The minimal extension of the SM symmetry $\mathrm{G}(2,1,3)$ that is needed to serve the purpose is once again given by the symmetry $\mathrm{G}(2,2,4)$, possessing the three features: (i) quark-lepton unification through $\mathrm{SU}(4)$-color, (ii) the L-R symmetric gauge structure $\mathrm{SU}(2)_{\mathrm{L}} \times \mathrm{SU}(2)_{\mathrm{R}}$, and (iii) the rationale for quantization of electric charge. In short, the three aesthetically desired features (i)-(iii) emerge simultaneously as necessary features to provide a unique answer to the single question posed above, without being assumed. Aesthetically, this particular aspect appeared to be quite appealing to us and suggested, starting in 1972-73, that the $\mathrm{SU}(4)$-color route to higher unification, embodied in the symmetry $\mathrm{G}(2,2,4)$, may well be used by nature at some level as being part of an ultimate picture. Fortunately, as we will see, such a route which of course includes extension of $\mathrm{G}(2,2,4)$ into a simple group, the smallest one being $\mathrm{SO}(10)$, can clearly be distinguished empirically from alternative ones such as those based on $\mathrm{SU}(5)$ devoid of SU(4)-color, through phenomena such as neutrino oscillations, leptogenesis, fermion masses and mixings, and even proton decay.

As an added desirable feature, since the symmetries $\mathrm{SU}(2)_{\mathrm{L}, \mathrm{R}}$ and the traceless vectorial SU(4)-color are individually free from the triangle anomalies, the gauge interactions of $\mathrm{G}(2,2,4)$, subject to $L-R$ discrete symmetry, can be chiral (as desired), yet anomaly-free, and importantly parity conserving (see discussion below)[53]. As alluded to before, this in turn accounts on symmetry-grounds for the non-trivial anomaly-cancelation within each fifteenmember family of the SM symmetry, since the extra RH neutrino of $\mathrm{G}(2,2,4)$ is a singlet of the SM.

Before discussing some of the additional major advantages of the symmetry $\mathrm{G}(2,2,4)$, let me mention one that is perhaps the most striking. 


\subsection{The Unwanted Right-Handed Neutrino}

Either one of the symmetries $S U(4)$-color or $S U(2)_{R}$ implies, however, that there must exist the right-handed counterpart $\left(\nu_{R}\right)$ of the left-handed neutrino $\left(\nu_{L}\right)$. This is because the $\mathrm{RH}$ neutrino $\left(\nu_{R}\right)$ is the fourth color partner of the RH up quark; and it is also the $S U(2)_{R}$ doublet partner of the RH electron. Thus, given the symmetry $G(2,2,4)$, one necessarily had to postulate the existence of an unobserved new member in each family - the right-handed neutrino.

This in turn meant, especially within the $\mathrm{SU}(4)$-color symmetry, that the neutrinos must be massive, like the quarks, posing the dilemma as to why they are so light. A natural resolution of this dilemma did emerge within the same $\mathrm{G}(2,2,4)$-framework within four years through the realization of the seesaw mechanism[14] and I will discuss the same shortly. Meanwhile, there was a strong prejudice, however, in the 1970's, and even through the 1990's till neutrino oscillations were discovered, against neutrinos being massive and therefore against the existence of the $\mathrm{RH}$ neutrinos as well. Given that the upper limits on neutrino masses were known to be so small: $\left(m\left(\nu_{\mathrm{e}}\right) / m_{\mathrm{e}} \lesssim 10^{-6}\right.$, and after the discoveries of $\nu_{\tau}$ and the top quark, $\left.m\left(\nu_{\tau}\right) / m_{\text {top }}<10^{-9}\right)$, many, perhaps most in the community, believed that they must be exactly massless 8 This is in fact what the two-component theory of the neutrino [56] as well as the standard electroweak model of particle physics [29] and the SU(5) grand unification model[10], possessing only LH neutrinos ( $\nu_{\mathrm{L}}$ 's), would naturally suggest 9] In this sense, the RH neutrino was regarded perhaps by most as an unwanted child (the ugly duckling) in the 1970's, and I faced much resistance from the community in my seminars as regards the unavoidable need for such an unwanted object.

My only defense at that time (1972-74) was, what appeared in my view, the grand beauty of the $\left[(2 \times 4)_{L}+(2 \times 4)_{R}\right]$ quark-lepton and L-R symmetric family-structure, which is naturally suggested by the $G(2,2,4)$ symmetry, explaining neatly the quantum numbers of all its members (see Eq.(3)). It is interesting that subsequent developments in theory and experiments, which were crucial, upheld this inner conviction. With the realization of the seesaw mechanism[14], and importantly the discovery of neutrino oscillations in 1998[12], the RH neutrino has turned out to be an asset (a beautiful swan) to understand neutrino oscillations as well as the observed baryon asymmetry of the universe. I will discuss these in Sec. 6. Let me now return to presenting the other key features of the symmetry $G(2,2,4)$.

The introduction of the $\mathrm{RH}$ neutrino requires that there be sixteen two-component fermions in each family, as opposed to fifteen for the standard model (SM) or the SU(5) symmetry. Subject to left-right discrete symmetry $(L \leftrightarrow R)$ which is natural to $G(2,2,4)$, all 16 mem-

\footnotetext{
${ }^{8}$ The extent to which this belief was ingrained among many leading physicists even in the 1990's may be assessed by an interesting remark by C.N. Yang at the 2002 Stony Brook conference on neutrinos [54. See also remarks in Ref. [55].

${ }^{9}$ This is barring, of course, possible contributions [57 to the Majorana mass of $\nu_{L}$ from lepton-number violating quantum gravity effects $\sim\left(v_{\mathrm{EW}}^{2} / M_{P l}\right) \sim(250 \mathrm{GeV})^{2} / 10^{19} \mathrm{GeV} \sim 10^{-5} \mathrm{eV}$, which are tiny, compared to the presently observed mass scales of atmospheric and even solar neutrino oscillations. As expressed elsewhere [58, this smallness of (possible) quantum gravity effects prompts one to regard the atmospheric and solar neutrino oscillations as clear signals for physics beyond what one may expect within the standard model combined with quantum gravity.
} 
bers of the electron family now became parts of a whole - a single left-right self-conjugate multiplet $F=\left\{F_{L} \oplus F_{R}\right\}$, where

$$
F_{L, R}^{e}=\left[\begin{array}{llll}
u_{r} & u_{y} & u_{b} & \nu_{e} \\
d_{r} & d_{y} & d_{b} & e^{-}
\end{array}\right]_{\mathrm{L}, \mathrm{R}}
$$

The multiplets $F_{L}^{e}$ and $F_{R}^{e}$ are left-right conjugates of each other transforming respectively as $(2,1,4)$ and $(1,2,4)$ of $\mathrm{G}(2,2,4)$; likewise for the muon and the tau families. The symmetry $S U(2)_{L, R}$ treat each column of $F_{L, R}^{e}$ as a doublet; while the symmetry $S U(4)$-color unifies quarks and leptons by treating each row of $F_{L}^{e}$ and $F_{R}^{e}$ as a quartet; thus lepton number is treated as the fourth color. A very special feature of the symmetry $\mathrm{G}(2,2,4)$ is now worth noting.

\subsection{Left-Right Symmetry in the Fundamental Laws:}

Because of the parallelism between the actions of $\mathrm{SU}(2)_{\mathrm{L}}$ and $\mathrm{SU}(2)_{\mathrm{R}}$ on the left and the right chiral fermions respectively, and because $\mathrm{SU}(4)$-color is vectorial, the gauge symmetry $\mathrm{G}(2,2,4)$, with the choice $g_{\mathrm{L}}^{(0)}=g_{\mathrm{R}}^{(0)}$, naturally opened the door for the novel and attractive concept that the laws of nature possess left $\leftrightarrow$ right discrete symmetry - i.e. parity invariance - at a fundamental level [59]10, that interchanges $\mathrm{F}_{\mathrm{L}}^{\mathrm{e}} \leftrightarrow \mathrm{F}_{\mathrm{R}}^{\mathrm{e}}$ together with $\mathrm{W}_{\mathrm{L}} \leftrightarrow \mathrm{W}_{\mathrm{R}}$. Subject to suitable restrictions on the Higgs system, the observed parity violation could then be interpreted as being a low-energy phenomenon arising entirely through a spontaneous breaking of the $\mathrm{L} \leftrightarrow \mathrm{R}$ discrete symmetry, which should disappear at appropriately high energies. Thus within this picture, (using Pauli's words) God is no longer "weakly left-handed"; left and right are treated on par at the fundamental level.

Briefly, I may mention that if one did not insist on quark-lepton unification through $\mathrm{SU}(4)$-color, parity invariance at a fundamental level can still be realized through the socalled "left-right symmetric (LRS)" model [9,59] based on the symmetry $\mathrm{G}(2,2,1,3)=$ $\mathrm{SU}(2)_{\mathrm{L}} \times \mathrm{SU}(2)_{\mathrm{R}} \times \mathrm{U}(1)_{\mathrm{B}-\mathrm{L}} \times \mathrm{SU}(3)^{\mathrm{c}}$, a sub-group of $\mathrm{G}(2,2,4)$. For a possible motivation for this sub-group, which might have been relevant in the pre-neutrino-oscillation era, see footnote 11 of Ref. [9]. Much work [60], especially in connection with having light $\mathrm{W}_{\mathrm{R}}$ 's which could possibly be observed at the LHC, has recently been carried out in the context of this minimal left-right symmetric (LRS) model.

I will return shortly to the relevance of having the $\mathrm{RH}$ neutrinos for an understanding of the neutrino masses. First, it is worth noting a few additional features of the symmetry $G(2,2,4)$ and its relationship to still higher symmetries.

1) The Charge Formula: The symmetry $G(2,2,4)$ introduces an elegant charge formula:

$$
Q_{e m}=I_{3 L}+I_{3 R}+(B-L) / 2
$$

that applies to all forms of matter (including quarks and leptons of all six flavors, Higgs and

\footnotetext{
${ }^{10}$ In the discussion to follow, the L-R discrete symmetry should thus be understood to accompany the symmetry $G(2,2,4)$, with or without being specified as such.
} 
gauge bosons) ${ }^{11}$ Note that the quantum numbers of all members of a family, including the weak hypercharge $Y_{W}=I_{3 R}+(B-L) / 2$, are now completely determined by the symmetry group $G(2,2,4)$ and the tranformation-property of $\left(F_{L} \oplus F_{R}\right)$. This is in contrast to the case of the SM for which the 15 members of a family belong to five disconnected multiplets, with unrelated quantum numbers. Quite clearly the charges $I_{3 L}, I_{3 R}$, and $B-L$ being generators of $S U(2)_{L}, S U(2)_{R}$, and $S U(4)^{c}$ respectively are quantized; so also then is the electric charge $Q_{e m}$.

2) An Intimate Link Between $S U(4)$-color and the L-R Symmetry: At this point, an intimate link between $S U(4)^{c}$ and $S U(2)_{L} \times S U(2)_{R}$ is worth noting. As remarked before, assuming that $S U(4)^{c}$ is gauged and requiring an explanation for the quantization of electric charge as above leaves one with no other choice but to gauge minimally the commuting symmetry $S U(2)_{L} \times S U(2)_{R}$ (rather than $\left.S U(2)_{L} \times U(1)_{I_{3 R}}\right)$. Likewise, assuming that $S U(2)_{L} \times S U(2)_{R}$ is gauged and again asking for a compelling reason for the quantization of electric charge dictate that one must minimally gauge the symmetry $S U(4)^{c}$ (rather than $\left.S U(3)^{c} \times U(1)_{B-L}\right)$. The resulting minimal gauge symmetry is then $G(224)=S U(2)_{L} \times S U(2)_{R} \times S U(4)^{c}$ that simultaneously achieves quantization of electric charge, quark-lepton unification and leftright symmetry. In short, the concepts of SU(4)-color and left-right gauge symmetry in its minimal form (symbolized by $S U(2)_{L} \times S U(2)_{R}$ ) become inseparable from each other, if one demands that there be an underlying reason for the quantization of electric charge. Assuming one automatically implies the other.

3) Universality of Weak Interactions: It is furthermore worth noting that the extension of the standard model symmetry to the level of the symmetry $\mathrm{G}(2,2,4)$ provides a compelling reason for why weak interactions are universal with respect to quarks and leptons, though strong interactions are not. This is because $\mathrm{SU}(2)_{\mathrm{L}}$, generating weak interactions, commutes with SU(4)-color (as it must for the sake of renormalizability) and thus treats all four colors representing quarks and leptons universally. Non-universality of strong interactions can be attributed, as mentioned before, to spontaneous breaking $\mathrm{SU}(4)$-color to $\mathrm{SU}(3)$-color $\times$ $\mathrm{U}(1)_{\mathrm{B}-\mathrm{L}}$ at high energies. These features, which were known from the 1940's and were essentially put in by hand to satisfy observations, thus found a rationale through quarklepton unification as in $\mathrm{G}(2,2,4)$.

4) The Two Simple Mass Relations: The symmetry SU(4)-color leads to two simple relations between the masses of quarks and leptons at the unification-scale $\mathrm{M}_{\mathrm{U}}$ :

$$
\begin{aligned}
m_{b}\left(M_{U}\right) & \approx m_{\tau} \\
m\left(\nu_{\text {Dirac }}^{\tau}\right) & \approx m_{\text {top }}\left(M_{U}\right)
\end{aligned}
$$

These two relations arise from the SU(4)-color-preserving leading entries in the fermion mass matrices which contribute to the masses of the third family [see Ref. [25] for a detailed discussion]. The sub-leading corrections that arise from SU(4)-color breaking in the (B-L) direction turn out to be important for the masses and mixings of only the first two families

\footnotetext{
${ }^{11}$ The 15th diagonal generator of $S U(4)$-color entering into the electric charge formula of Ref. 9 is naturally proportional (as per Eq. (11)) to the charge $\left(\mathrm{B}_{\mathrm{q}}-3 \mathrm{~L}\right)$ where $B_{q}=$ quark number. It was pointed out in Ref. 61] that this can be expressed in terms of the more familiar charge $(B-L)$, since $B_{q}=3 \mathrm{~B}$, resulting in Eq. (4).
} 
[25], and that, of course, goes well with observations. This is discussed in Sec. 5. Now, of the two relations given above, the first is successful empirically. As we will see in Sec. 4, the second is crucial to the success of the seesaw formula for $m\left(\nu_{\mathrm{L}}^{\tau}\right)$ and thereby for the observed $\delta m^{2}(\nu)_{23}$.

5) B-L as a Local Symmetry: The symmetry SU(4)-color contains B-L as a generator, which provides some essential benefits. First, with B-L remaining intact at least upto the unification scale $\mathrm{M}_{\mathrm{U}} \approx 2 \times 10^{16} \mathrm{GeV}$, it serves to protect the $\mathrm{RH}$ neutrinos from acquiring a Planck or string-scale $\left(\sim 10^{18} \mathrm{Gev}\right)$ Majorana mass through quantum gravity effects. ${ }^{12}$ Following limits from the Eötvos-type experiments, however, one can argue that B-L must be violated at some scale $\mathrm{M}_{\mathrm{B}-\mathrm{L}}$ (considerations based on MSSM gauge coupling unification, that predicts the weak angle successfully, would in fact suggest that $\mathrm{M}_{\mathrm{B}-\mathrm{L}} \approx \mathrm{M}_{\mathrm{U}} \approx 2 \times 10^{16} \mathrm{GeV}$, see Secs. 5 and 6 ). Now $\mathrm{M}_{\mathrm{B}-\mathrm{L}}$ sets the scale for the (superheavy) Majorana masses of the $\mathrm{RH}$ neutrinos, and thereby plays a crucial role in determining the masses of the light LH neutrinos via the seesaw formula (see Sec. 6). Furthermore, spontaneous breaking of B-L allows one to implement the mechanism of baryogenesis via leptogenesis in the presence of the elctroweak sphaleron effects which wipe out any (B-L)-conserving matter-antimatter asymmetry (see Sec. 6).

In brief, viewed against the background of particle physics in 1972-73, the symmetry $G(2,2,4)$ brought some attractive features for the first time. These include:

(i) Unification of all 16 members of a family within one left-right self-conjugate multiplet, with a neat explanation of all their quantum numbers;

(ii) Quantization of electric charge, with $\mathrm{Q}_{\mathrm{e}^{-}}+\mathrm{Q}_{\mathrm{p}}=0$;

(iii) Quark-lepton unification through $S U(4)$-color;

(iv) A rationale for the co-existence of the weak, electromagnetic and strong forces, which, together with the features listed above, set the stage for their unity [7,10] possessing $\mathrm{SU}(4)$-color, within a symmetry like $S O(10)[11]$.

(v) A compelling reason for the universality of the weak interactions with respect to quarks and leptons;

(vi) Conservation of parity at a fundamental level [59];

(vii) The RH neutrino as a compelling member of each family;

(viii) B-L as a local symmetry;

(ix) A rationale for the now-successful mass-relations 5) and 6); and

(x) The right set-up, due to (vii) and (viii), for implementing baryogenesis via leptogenesis.

\footnotetext{
${ }^{12}$ Such an ultraheavy Majorana mass $\left(\sim 10^{18} \mathrm{GeV}\right)$ for the RH neutrino would be unacceptable because it would lead to too tiny a mass $\left(<10^{-4} \mathrm{eV}\right)$ for even the heaviest Dirac mass $\sim 200 \mathrm{GeV}$ for any neutrino species through the seeaw formula.
} 
These ten features, together with the two intriguing predictions mentioned below, constitute the hallmark of the symmetry $G(2,2,4)$, fulfilling major aesthetic ((i)-(vi)) as well as practical ((vii)-(x)) needs. As mentioned before, the three distinguishing features of $G(2,2,4)$ - i.e. the existence of the $\mathrm{RH}$ neutrinos and B-L as a local symmetry, together with the mass-relation 6), now seem to be essential to understand naturally the tiny massscales observed in neutrino oscillations, in the context of the seesaw mechanism, and to implement the mechanism of baryogenesis via leptogenesis. This will be discussed in more detail in Secs. 5 and 6.

\section{(xi) Existence of Magnetic Monopoles}

In addition to the features listed above, a deep and crucial consequence of the idea that electromagnetism has its origin within a spontaneously broken non-Abelian gauge symmetry ( semi-simple or simple), so that electric charge would be quantized, is the existence of topological 't Hooft-Polyakov magnetic monopoles [62], with masses $\sim \mathrm{M}_{\mathrm{X}} / \alpha_{\mathrm{X}}$, where $\mathrm{M}_{\mathrm{X}}$ is the mass of the gauge boson(s) associated with the relevant symmetry breaking and $\alpha_{\mathrm{X}}$ is the corresponding gauge coupling. The symmetry $G(2,2,4)$, being the first realistic example of such a symmetry, thus necessarily predicts the existence of magnetic monopoles. As a distinction, the $G(2,2,4)$ - monopole would carry two units of Dirac magnetic charge $\mathrm{g}_{\mathrm{D}}=2 \pi / \mathrm{e}$, in contrast to one unit for the $\mathrm{SU}(5)$-monopole [63].

An interesting point here is the following. Dirac observed that the existence of even a single magnetic monopole (in his case, point-like) would imply quantization of electric charge [64. A satisfactory quantum theory of such Dirac monopoles is, however, lacking. The argument is now reversed in the context of higher unification symmetries like $G(2,2,4)$ or its extensions to simple groups. These theories necessarily quantize electric charge and thereby predict the existence of topological monopoles which are fully consistent with relativistic quantum theory. Based on the simplest interpretations of the observed gauge coupling unification and the mass-scales of neutrino oscillations, discussed in Secs.3 and 6, a grandunified symmetry like SUSY $S O(10)$ or a string-unified $G(2,2,4)$ symmetry ( see Sec. 3 ) is expected to break in one step at the unification-scale $\mathrm{M}_{\mathrm{U}} \sim 2 \times 10^{16} \mathrm{GeV}$ to the standard model. In this case, the associated magnetic monopoles (to be called GUT-monopoles) would be superheavy with masses $\sim$ few $\times 10^{17} \mathrm{GeV}$. These monopoles are thus too heavy to be produced by accelerators in the conceivable future.

At the same time, considering that the only known consistent quantum theory of a magnetic monopole is the topological one, as noted above, the discovery of a single magnetic monopole ( either superheavy or medium-heavy (see below)) would provide a conclusive evidence ( stronger than any other) for the existence of a GUT or GUT-like symmetry at high energies. In other words, magnetic monopole is a very precious property of GUT.

Despite their heaviness, in the context of a spontaneously broken GUT or GUT-like symmetry, one expects that these beautiful objects must have been produced in abundance in the very early universe. Unfortunately for their detectability now, though "fortunately" for our own existence, it seems most likely that, soon after being created, they were super-diluted by the inflationary expansion of the universe [65], for which the theoretical motivations and 
empirical evidence are now strong. The severe dilution of GUT- monopoles, expected within the inflationary picture, with the number of e-folds being greater than about 50 or so, is of course in accord with the stringent upper limits on the relic cosmic monopole flux which have been set by a variety of sensitive searches for a wide range of the monopole mass ( see the two papers in Ref. [66] for a review of the current status and references there in). Thus, within the inflationary picture, one would expect that the superheavy GUT monopoles have been inflated away beyond the level of observability.

There is, however, the possibility that relic monopoles of intermediate mass-scales $\sim$ $10^{14} \mathrm{GeV}$ (say), subject to a reduced number of e-foldings ( 30 or so) may still be around so as to be detectable through improved searches. Such a possibility would arise if a symmetry like $S O(10)$ breaks in two steps to the SM symmetry ( instead of one) via the symmetry $G(2,2,4)$, with the latter breaking at an intermediate scale of $\sim 10^{13} \mathrm{GeV}$ (say) to the SM. In this case, the superheavy monopoles associated with $S O(10)$ - breaking would be inflated away, but those associated with the subsequent step of $G(2,2,4)$-breaking, subject to a milder e-folding (of $\sim 30$, say), can survive in accord with all cosmological constraints and current empirical limits so as to be detectable through improved searches. Consistency of such a scenario with the desired cosmological parameters (including the spectral index $n_{s}$ and the tensor to scalar ratio r), and also with the limits from the monopole searches [66], has been shown within a non-supersymmetric inflationary $S O(10)$ model [67.

Now, as alluded to above, based on the simplest interpretation of the observed gauge coupling unification that leads to a successful prediction for the weak angle (Sec. 3) and an understanding of the mass-scales of the neutrino oscillations (Sec. 6), it is the one-step breaking of supersymmetric $S O(10)$ to the SM that is favored over the two-step breaking scenario. Nevertheless, given the importance of the discovery of a magnetic monopole as noted above, allowance should be made for the lack of certainty in our understanding of the pattern of GUT symmetry-breaking. In matter such as this, we must let experiment alone be the guide. Thus searches for magnetic monopoles covering a wide range of masses need to be pursued so as to push the current limits as close to the threshold of observability as possible, unless monopoles are discovered in the process.

Let me now turn to one of the most novel features that emerged as a consequence of attempts at higher unification. The symmetry $G(2,2,4)$ needing a spontaneous violation of B-L introduced a new line of thinking leading to a questioning of the conservations of baryon and lepton numbers $[8]^{13}$, which were otherwise held sacred owing to the extraordinary stability of the proton. Such a questioning in turn evolved in the context of unification-ideas going beyond that of the symmetry $G(2,2,4)$ leading to the hallmark prediction of grand unification - i.e. proton decay [8,10]. This is briefly noted below, together with other fundamental processes violating B and/or L. Proton decay will be discussed in more detail in Sec. 7.

\footnotetext{
${ }^{13}$ The case of spontaneous violation arises because B-L is gauged in $G(2,2,4)$. Now, a massless gauge particle coupled to any linear combination of $\mathrm{B}$ and $\mathrm{L}$ (which is gauged) must acquire a mass through SSB in order to conform with the limits from the Eötvos type experiments. The corresponding charge (B and/or L) must then be violated spontaneously.
} 


\subsection{Non-Conservations of B and L: Proton Decay as a Generic Feature}

As mentioned before, B-L being a local symmetry in $G(2,2,4)$, the combination (B-L) and therefore $\mathrm{B}$ and/or L must be violated spontaneously 13 . It was recognized that this feature is a reflection of a more general phenomenon involving non-conservations of baryon and lepton numbers which are most likely to occur in unified gauge theories [8,10], including those going beyond the symmetry $G(2,2,4)$, that unify quarks and leptons as well as their three basic forces. Depending upon the nature of the gauge symmetry and the multiplet structure, the violations of $\mathrm{B}$ and/or $\mathrm{L}$ could be spontaneous, as is the case for non-conservation of $\mathrm{B}-\mathrm{L}$ in $\mathrm{SU}(4)$-color, and those of $\mathrm{B}$ and $\mathrm{L}$ in the maximal one-family symmetry $\mathrm{SU}(16)$ which gauges both B and L Ref. 68. Alternatively, the violations could be explicit; that is what happens for the subgroups of $\mathrm{SU}(16)$, like $\mathrm{SU}(5)[10$ ] and $\mathrm{SO}(10)$ [11], for which the gauge boson interactions violate B and L. One way or another, baryon and lepton number conservations cannot be absolute in the context of such higher unification.

The simplest and most dramatic consequence of such non-conservation is proton decay $(\Delta B \neq 0, \Delta L \neq 0)$, which as will see provides an indispensable tool to probe physics at truly high energies $\sim 10^{16} \mathrm{GeV}$, for proton decaying via the canonical $\mathrm{d}=6(\Delta(B-L)=0)$ decay modes, such as: $p \rightarrow e^{+} \pi^{\circ}$ and $p \rightarrow \bar{\nu} K^{+}$.

The other fundamental "processes" are the lepton-number violating Majorana masses of the RH and LH neutrinos $(\Delta B=0,|\Delta L|=2)$, which are relevant to an understanding of the neutrino masses via the seesaw mechanism, and in a related context the nutrinoless double beta decay $\left(n n \rightarrow p p e^{-} e^{-}\right)[72,73]$, both of which arise naturally within $G(2,2,4)$ and $S O(10)$ symmetries. Based on our current understanding of masses and mixings of all fermions including neutrinos in the context of such unification symmetries, the neutrinoless double beta decay should occur at some level and could quite plausibly lie within the realm of observation in several ongoing or next-generation experiments [73].

The third equally fundamental process violating only baryon number is $n-\bar{n}$ oscillation [74,75] $\left(|\Delta B|=2, \Delta L=0\right.$ ) which probes into physics typically at energy-scales of $\sim 10^{5} \mathrm{GeV}$ (or possibly lower). Several promising models have been constructed within symmetries like $G(2,2,4), \mathrm{SO}(10)$ and $G(2,2,1,3)$ (see e.g. Ref. [75]) which suggest that $n-\bar{n}$ oscillation could well be discovered if the current sensitivity can be improved by one to two orders of magnitude, reaching free $n-\bar{n}$ oscillation time $\sim 10^{9}-10^{10} \mathrm{~s}$.

Now any of these three processes, if seen, would be a breakthrough in particle physics, shedding light on fundamental physics at a deeper level. Thus the need for an improved sensitive study of each of these three processes cannot be overemphasized.

In this talk, I will focus primarily on three aspects: (i) gauge coupling unification (Sec. 3), (ii) an understanding of the mass-scale(s) of neutrino oscillations (Secs. 5 and 6), and (iii) proton decay (Sec. 7). These turn out to be intimately linked in the context of a promising class of grand unification models. As we will see, proton decay arises as a generic feature of most grand unification models if one seeks for the simplest realization of gauge coupling unification that permits a successful prediction of the weak angle (see Sec. 3), as well as an understanding of the mass-scale of the observed atmospheric neutrino oscillation (Sec. 6 
). One can in fact argue, within a class of well-motivated models of grand unification that proton decay should occur at accessible rates, with a lifetime bounded above by about $10^{35}$ years, for proton decaying into $\mathrm{e}^{+} \pi^{0}$, and a lifetime of less than about $(1-8) \times 10^{34}$ years for proton decaying into $\bar{\nu} \mathrm{K}^{+}$. These lifetimes are within factors of $5-10$ above the current limits on proton lifetimes coming from Super-Kamiokande 76,77, raising hopes that proton decay should most likely be discovered in the planned DUNE and/or HyperKamiokande experiments.

I next consider the idea of extending the symmetry $G(2,2,4)$ to simple groups which serve to unify the three forces.

\subsection{Going Beyond $\mathrm{G}(2,2,4): S O(10)$ and $S U(5)$}

To realize the idea of a single gauge coupling governing the three forces, [7, 10] one must embed the standard model symmetry, or $\mathrm{G}(2,2,4)$, in a simple or effectively simple group (like $\mathrm{SU}(\mathrm{N}) \times \mathrm{SU}(\mathrm{N})$ ). Several examples of such groups have been proposed. Howard Georgi and Sheldon Glashow proposed the first such group SU(5) [10] which contains the standard model symmetry, but not $\mathrm{G}(2,2,4)$. Following the discovery of asymptotic freedom of nonAbelian gauge theories 41] and the suggestion of SU(5), Georgi, Helen Quinn and Weinberg showed how renormalization effects, following spontaneous breaking of the unification symmetry, can account (to a good approximation) for the observed disparity between the three gauge couplings at low energies[18]. Subsequently, such a unification of the gauge couplings was shown to hold to a much better accuracy in the context of low-energy supersymmetry [19] and more precise measurements of the gauge couplings at LEP. Each of these contributions played a crucial role in strengthening the ideas of higher unification.

Now one can retain all the aesthetic and practical advantages ((i)-(x), listed in Sec. 2.5) of the symmetry group $\mathrm{G}(2,2,4)$ and in addition achieve gauge coupling unification, if one extends the symmetry $\mathrm{G}(2,2,4)$ (which is isomorphic to $S O(4) \times S O(6)$ ) minimally into the simple group $S O(10)[11$. As a historical note, it is worth noting, however, that leaving aside gauge coupling unification[7]10, all the attractive features of $S O(10)$, arise entirely at the level of the symmetry $\mathrm{G}(2,2,4)$, subject to the L-R discrete symmetry[59], and were introduced as such [8,9], well before $S O(10)$ was proposed [11]. These include in particular: members of a family belonging to a single multiplet, quantization of electric charge, the existence of the right-handed neutrino, $B-L$ as a local symmetry, and quark-lepton unification through $S U(4)$-color ( see the list in Sec. 2.5 ). The advantages of the last three features in understanding neutrino masses and implementing baryogenesis via leptogenesis will be discussed in Sec. 6.

The symmetry $S O(10)$ of course fully preserves these features ${ }^{14}$ because it contains $\mathrm{G}(2,2,4)$ as its maximal subgroup, with the latter being non-Abelian. It is furthermore remarkable that $S O(10)$ preserves even the left-right self-conjugate sixteen-component familystructure of $\mathrm{G}(2,2,4)$ by using the set $F=\left(F_{L} \oplus\left(F_{R}\right)^{c}\right)$ as its spinorial representation.

\footnotetext{
${ }^{14}$ In the context of $S O(10)$, the L-R discrete symmetry associated with $\mathrm{G}(2,2,4)$ is replaced by an equivalent generalized charge conjugation symmetry, which is a gauge symmetry in $S O(10)[78$.
} 
Interestingly, following the discovery of neutrino oscillations [12]13], this sixteen-component family-structure has emerged as being not too small ( unlike the $\overline{5}+10$ of SU(5)) and not too big ( unlike the 27 of $E_{6}$ [79] with several unobserved members), but just right, possessing the extra $R H$ neutrino. The latter, added to the $\overline{5}+10$ of $S U(5)$, makes a family of sixteen, which is special to both $\mathrm{G}(2,2,4)$ and $S O(10)$.

In contrast to the extension of $\mathrm{G}(2,2,4)$ to $S O(10)$ or $E_{6}$, if one wished to extend only the SM symmetry $\mathrm{G}(2,1,3)$ to a simple group, the minimal such extension would be $S U(5)[10]$. In the 1970s, long before the discovery of neutrino oscillations, the symmetry $S U(5)$, being the smallest simple group possessing the SM symmetry, served the important purpose of demonstrating the ideas of grand unification simply. It, however, does not contain $\mathrm{G}(2,2,4)$ as a subgroup. As such, except for the features of quantization of electric charge (feature (ii)), universality of weak interactions ((iv)), and b- $\tau$ mass-equality of Eq. (5), $S U(5)$ does not possess the other advantages of of $\mathrm{G}(2,2,4)$ listed in Sec. 2.5, including: (a) the $\mathrm{RH}$ neutrino , (b) $B-L$ as a local symmetry, and (c) the mass-relation of Eq. (6), based on $S U(4)$-color ${ }^{15}$ As I will discuss in Sec. 6, the last three features play crucial roles in providing an understanding of neutrino masses and in implementing baryogenesis via leptogenesis. Furthermore $S U(5)$ splits members of a family (not including $\nu_{R}$ or $\left(\nu_{R}\right)^{c}$ ) into two multiplets: $\overline{5}+10$, and it violates parity, like the SM, manifestly.

Comparing $\mathrm{G}(2,2,4)$ with $S O(10)$, as mentioned above, $S O(10)$ possesses all the features (i) to $(\mathrm{x})$ of $\mathrm{G}(2,2,4)$ listed in Sec. 2.5 , but in addition it offers gauge coupling unification. I should, however, mention at this point that the perspective on coupling unification and proton decay changes considerably in the context of supersymmetry and superstrings. In balance, a string-unified $\mathrm{G}(2,2,4)$ symmetry offers some advantages over a string-derived $S O(10)$ symmetry in $4 \mathrm{D}$, while the reverse is true as well. Thus, it seems that a definite choice of one over the other is hard to make at this point. This will be discussed briefly in the next section.

\section{Gauge Coupling Unification}

It has been recognized from the 1970's that the concept of higher unification - now commonly called Grand Unification (abbreviated as GUT) - has three dramatic consequences:

(i) a meeting of the three gauge couplings at a high scale;

(ii) the prediction of nonzero but superlight neutrino masses and thereby of neutrino oscillations, which is a special feature of a sub-class of grand unification symmetries, in particular those containing $\mathrm{SU}(4)$-color, like $\mathrm{SO}(10)$ or $\mathrm{E}_{6}$ or a string-derived $\mathrm{G}(2,2,4)$ symmetry (see below), in the context of the seesaw mechanism; and last but not least;

(iii) proton decay.

\footnotetext{
${ }^{15}$ Like $S O(10), S U(5)$ does possess, however, fermion to anti-fermion gauge transformations which are absent in $\mathrm{G}(2,2,4)$.
} 

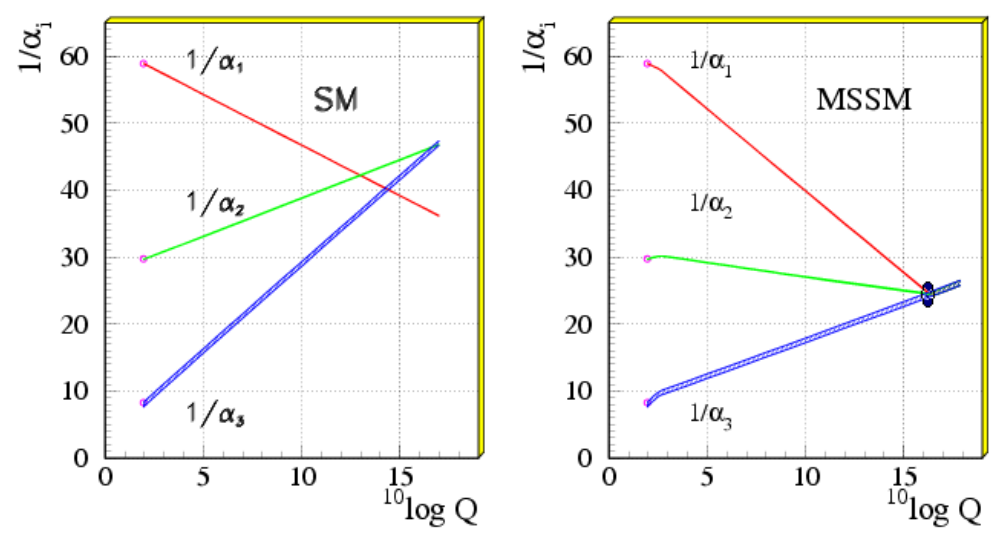

Figure 1: Evolution of the three gauge couplings $\alpha_{i}$ with momentum $Q$ : Standard Model (left panel) and Minimal Supersymmetric Standard Model (right panel)

I will discuss the empirical verification of the first two features and the consequent implications in this and the next section. The third feature - proton decay — which now constitutes the missing piece of evidence for grand unification is discussed in Sec. 7.

It has been known for some time that the precision measurements of the standard model coupling constants (in particular $\sin ^{2} \theta_{\mathrm{W}}$ ) at LEP put severe constraints on the idea of grand unification. Owing to these constraints, the non-supersymmetric minimal $S U(5)$, and for similar reasons, the one-step breaking minimal non-supersymmetric $S O(10)$-model as well, are now excluded [80]. For example, minimal non-supersymmetric $S U(5)$ predicts: $\left.\sin ^{2} \theta_{W}\left(m_{Z}\right)\right)\left.\right|_{\overline{M S}}=0.214 \pm 0.004$, where as current experimental data show: $\sin ^{2} \theta_{W}\left(m_{Z}\right)_{\text {expt }}{ }^{L E P}=$ $0.23153 \pm 0.00016$ [81]. The disagreement with respect to $\sin ^{2} \theta_{W}$ is reflected most clearly by the fact that the three gauge couplings $\left(g_{1}, g_{2}\right.$ and $\left.g_{3}\right)$, extrapolated from below, fail to meet by a fairly wide margin in the context of minimal non-supersymmetric $S U(5)$ (see left panel of Fig. 11).

But the situation changes radically if one assumes that the standard model is replaced by the minimal supersymmetric standard model (MSSM), above a threshold of about $1 \mathrm{TeV}$, which is motivated independently by requiring naturalness in the understanding of the Higgs mass 21]. Furthermore, subject to the assumption of R-parity or matter-parity conservation, which serves to avoid dangerous $\mathrm{d}=4$ proton-decay operators, low-energy supersymmetry provides the lightest supersymmetric particle (LSP) as a natural candidate for the cold dark matter. With low-energy supersymmetry, the three gauge couplings are found to meet [19] to a very good approximation (See Fig. 1, right panel) at a scale given by

$$
M_{U} \approx 2 \times 10^{16} \mathrm{GeV} \quad(\mathrm{MSSM} \text { or } \operatorname{SUSY} \mathrm{SU}(5))
$$

This dramatic meeting of the three gauge couplings, or equivalently the excellent agreement of the MSSM-based prediction of $\sin ^{2} \theta_{W}\left(m_{Z}\right)_{\mathrm{Th}}=0.2315 \pm 0.003$ [1980] with the observed value given above, provides a strong support for the ideas of both grand unification and low-energy supersymmetry, as being relevant to physics at short distances.

The simplest interpretation of the observed meeting of the three gauge couplings at 
the scale $M_{U}$, that preserves the successful prediction of the weakangle $\sin ^{2} \theta_{W}$, is that a supersymmetric grand unification symmetry (often called GUT symmetry), like SU(5) or $\mathrm{SO}(10)$, breaks spontaneously in one step, (as opposed to possible multiple steps, relevant to $\mathrm{SO}(10))$ at the scale $M_{U}$ into the standard model symmetry $G(2,1,3)$.

Before discussing possible alternative interpretation of the observed meeting of the gauge couplings in the context of string/M theory, it is worthnoting that, unlike SU(5), a symmetry like $\mathrm{SO}(10)$ can in general break spontaneously in multiple steps (two or more) to the SM via a symmetry like $G(2,2,4)$ (see e.g. Refs. [82] and [83], and two recent works [84,85]) involving intermediate scale(s) of symmetry breaking. In these cases, even without supersymmetry, gauge coupling unification can be made to work by choosing appropriately the intermediate scale(s) of symmetry breaking. But in such cases, the weak angle is no longer a prediction; it needs to be used as an input to fix the intermediate scale(s).16 Thus, in these cases, the success of the simplest interpretation of the observed meeting of the gauge couplings in predicting the weak angle in excellent agreement with experiments, which is realized in the context of an one-step breaking of supersymmetric $\mathrm{SO}(10)$, will have to be regarded only as an accident. Remarkably enough, an identical conclusion emerges in attempting to have a natural understanding of the mass-scale of the atmospheric neutrino oscillation (see Sec. 6). In short, two independent phenomena - gauge coupling unification and the mass-scale of the atmospheric neutrino oscillation - seem to lead to one and the same conclusion favoring the simplest picture of the one-step breaking of a SUSY GUT Symmetry (like $\mathrm{SO}(10))$ or a string-unified $G(2,2,4)$ symmetry (see below)) to the SM symmetry at a high scale $M_{U} \approx 2 \times 10^{16} \mathrm{GeV}$.

\subsection{SUSY SO(10) Versus a String-Unified G(2,2,4) Symmetry in 4D}

Now, the simplest interpretation, mentioned above, would require a SUSY GUT-symmetry, like $\mathrm{SO}(10)$, to be effective at and above the unification-scale $M_{U}$ in $4 \mathrm{D}$. An alternative interpretation that would permit a supersymmetric non-GUT symmetry like $G(2,2,4)$ breaking in one step into the SM symmetry at the unification-scale $M_{U}$ is, however, possible in the context of string or $\mathrm{M}$ theory, which seems to be needed to unify all the forces of nature including gravity and also to obtain a good quantum theory of gravity. This is because, even if the effective symmetry in four dimensions emerging from a higher dimensional string theory is non-simple, like $G(2,2,4)$ or $G(2,1,4)$, or even the SM symmetry $G(2,1,3)$, string theory can still ensure familiar unification of the gauge couplings at the string scale. In this context, one could, therefore, first ask: can the effective symmetry below the string-scale in $4 \mathrm{D}$ be as small as the SM symmetry $G(2,1,3)$ ? I would argue (see Sec. 5 and 6 ) that attempts to understand naturally the neutrino mass-scales utilizing the seesaw mechanism, and to implement the mechanism of baryogenesis via leptogenesis successfully, provide an answer to the contrary. Both suggest clearly that the effective symmetry in $4 \mathrm{D}$, below the string scale, should

\footnotetext{
${ }^{16}$ The case of Ref. 84 is somewhat of an exception in this regard because the single intermediate scale associated with the breaking of the $G(2,2,4)$ symmetry to the SM is identified with the Peccei-Quinn symmetry breaking scale. But, even in this case, intermediate-scale breaking of B-L does not go well with attempts to obtain a natural understanding of the mass-scale of the atmospheric neutrino oscillation (see Sec 6).
} 
minimally contain either the $G(2,2,4)$ or its close relative $G(2,1,4)=S U(2)_{L} \times I_{3 R} \times S U(4)^{c}$ symmetry. This is because, as mentioned before, B-L should survive at least up to the unification scale $M_{U}$ so as to protect the RH neutrinos from acquiring Majorana masses of the string or Planck scale. $\sqrt{17}$ Furthermore, the full SU(4)-color symmetry, that contains B-L, is needed to ensure the two Dirac mass-relations given in Eqs. (5) and (6). The first is empirically successful, while the second is needed crucially for the success of the seesaw mechanism (see Sec. 6).

While keeping $G(2,1,4)$ in the picture, to simplify discussion, I will thus proceed to consider the two alternatives of a (hopefully) realistic string/M-theory solution possessing either an effective $G(2,2,4)$ symmetry or the GUT-symmetry $\mathrm{SO}(10)$, both with supersymmetry, emerging at the string-scale in 4D. Needless to say, among the vast landscape of string/M-theory solutions, which are essentially perturbative in nature, there is no criterion of selectivity at present, and identifying a completely realistic solution, even with only the SM symmetry in 4D, remains a challenge. Thus, at present, combining a top-down with a bottom-up approach, hoping that the two would meet at just the desired structure, seems to be the best way. Within the vast landscape of string solutions, there do exist, however, promising semi-realistic solutions containing an effective $G(2,2,4)$ symmetry in $4 \mathrm{D}$ [86,87,88, with three generations, and some of them with the right set of Higgs-like multiplets to do the desired symmetry breaking in 4D ( see e.g. B. Assel et al. in [86] and Refs. 87] and [88]). Semi-realistic string-derived $\mathrm{SO}(10)$ solutions also exist [89] (though these have not been studied as much as those for the case of $G(2,2,4)$ ). While the emergence of such solutions from within a fundamental theory is encouraging, much work still needs to be done to realize the essential ingredients in $4 \mathrm{D}$ within one such solution. Thus at present, the existence of a completely ( or close to) realistic string-G $(2,2,4)$ or string-SO(10) solution in $4 D$, with the desired spectrum and couplings, can only be regarded as an assumption. In view of the strong empirical motivations for either one of these two symmetries being effective in $4 \mathrm{D}$, and the theoretical motivations for the string/M theory being the mother theory, consequences of such an assumption seems worth pursuing, and I will do so in the following.

Let me now return to the question of a consistency that arises for a non-GUT $G(2,2,4)$ like string solution in 4D. While string theory will ensure gauge coupling unification (i.e. $\left.g_{2 \mathrm{~L}}=g_{2 \mathrm{R}}=g_{4}\right)$ at the string-scale, one needs to account for the mismatch by about a factor of 20 between the MSSM unification scale $M_{U}$ (given above), and the string-unification scale, given by $M_{s t} \approx g_{s t} \times 5.2 \times 10^{17} \mathrm{GeV} \approx 3.6 \times 10^{17} \mathrm{GeV}$ (Here we have put $\alpha_{s t}=$ $\left.\alpha_{G U T}(\mathrm{MSSM}) \approx 0.04\right)[90$. Possible resolutions of this mismatch have been proposed. These include:

(i) utilizing the idea of string-duality 91] which allows a lowering of $M_{s t}$ compared to the value shown above, or alternatively

(ii) allowing the possibility of highly anisotropic string compactification in which one or two of the compact radii are extremely large compared to the others 92 ,

(iii) using the idea of a semi-perturbative unification that assumes the existence

\footnotetext{
${ }^{17}$ Such an ultraheavy Majorana mass $\left(\sim 10^{18} \mathrm{GeV}\right)$ for the RH neutrino would be unacceptable because it would lead to too tiny a mass $\left(<10^{-4} \mathrm{eV}\right)$ for even the heaviest LH neutrino through the seeaw formula.
} 
of two vector-like families, transforming as $(16+\overline{16})$, at the $\mathrm{TeV}$-scale. The latter raises $\alpha_{G U T}$ to about $0.25-0.3$ and simultaneously $M_{U}$, in two loop, to about $(1 / 2-2) \times 10^{17} \mathrm{GeV}$ [93].

(iv) Assuming a Few Extra Multiplets at GUT-scale: A fourth possibility [94, which has not appeared in a published form yet, would arise if a supersymmetric $G(2,2,4)$ solution emerges from string theory in $4 \mathrm{D}$, possessing a few extra Higgs-like multiplets (as mentioned below) having GUT-scale masses; most of these will be needed to break the symmetry $\mathrm{G}(2,2,4)$ at the GUT-scale and/or give appropriate masses and mixings to the fermions any way. These extra multiplets are in addition, of course, to the familiar lowenergy spectrum of MSSM possessing: (a) the three generations of quarks and leptons, (b) one light (massless) multiplet $(2,2,1)_{\mathrm{H}}$ of $G(2,2,4)$ which contains $\mathrm{H}_{\mathrm{u}}$ and $\mathrm{H}_{\mathrm{d}}$ of MSSM and serves to break the EW symmetry, and (c) their superpartners. The assumed GUTscale multiplets, that would serve the desired purpose of GUT-scale symmetry-breaking, while preserving L-R discrete symmetry and accounting for the mismatch between $\mathrm{M}_{\mathrm{U}}$ and $\mathrm{M}_{\mathrm{st}}$ (mentioned above), are given by the set:

$$
\begin{aligned}
\mathrm{H}_{\mathrm{GUT}}= & \mathrm{H}_{\mathrm{SSB}}+\mathrm{H}_{\text {extra }}^{\prime} \\
= & {\left[(1,1,15)_{\mathrm{H}}+\left\{(2,1,4)_{\mathrm{H}}+(1,2, \overline{4})_{\mathrm{H}}\right\}_{\mathrm{A}}+\left\{(2,1, \overline{4})_{\mathrm{H}}+(1,2,4)_{\mathrm{H}}\right\}_{\mathrm{B}}\right]_{\mathrm{SSB}} } \\
& +\left[(1,1,15)_{\mathrm{H}}^{\prime}+(2,2,1)_{\mathrm{H}}^{\prime}\right]_{\text {extra }}
\end{aligned}
$$

Note that, of these extra multiplets, the $(1,1,15)_{\mathrm{H}}$ is the analog of $45_{\mathrm{H}}$ of $\mathrm{SO}(10)$ and the sub-sets $\mathrm{A}$ and $\mathrm{B}$ correspond precisely to $16_{\mathrm{H}}$ and $\overline{16}_{\mathrm{H}}$ of $\mathrm{SO}(10)$ respectively, while $\left(2,2,1^{\prime}\right)_{\mathrm{H}} \subset 10_{\mathrm{H}}^{\prime}$ of $\mathrm{SO}(10)$. The VEVs of $\left\{(1,2,4)_{\mathrm{H}}+(1,2, \overline{4})_{\mathrm{H}}\right\}$ of GUT-scale are in fact needed to break the gauge symmetry $G(2,2,4)$ to the SM, while preserving supersymmetry; and the corresponding L-R conjugate multiplets $\left\{(2,1,4)_{\mathrm{H}}+(2,1, \overline{4})_{\mathrm{H}}\right\}$ should be present, if L-R discrete symmetry survives compactification, which we assume. This is the exact analog of the roles of $\left\{16_{\mathrm{H}}+\overline{16}_{\mathrm{H}}\right\}$ for breaking $\mathrm{SO}(10)$ (see Sec. 4). Also one of the two $(1,1,15)_{\mathrm{H}}$ $\left(\subset 45_{\mathrm{H}}\right.$ of $\left.\mathrm{SO}(10)\right)$ having a GUT-scale VEV along the $(\mathrm{B}-\mathrm{L})$-direction is needed to introduce the observed (B-L)-dependence in fermion masses and mixings, through the use of higher dimensional operators (see Sec. 5).

In this sense, all the multiplets listed in the first square bracket on the RHS of Eq.(8) are in fact needed, minimally, to implement necessary symmetry breaking and/or to give desired masses and mixings to the fermions, while preserving supersymmetry and L-R symmetry. Only the two multiplets- i.e. the $(1,1,15)_{\mathrm{H}}^{\prime}$ and the $(2,2,1)_{\mathrm{H}}^{\prime}$ - in the second square bracket of Eq. (8) are really "extra". For comparison, it may be noted that analogous Higgs multiplets like $16 \mathrm{H}, \overline{16}_{\mathrm{H}}$ and $45_{\mathrm{H}}$ are needed also for the case of an intact SUSY SO(10) in 4D to serve identical purposes. Furthermore, for the latter, a few additional multiplets like a pair of $\left(16_{\mathrm{H}}^{\prime}\right.$ and $\left.\overline{16}_{\mathrm{H}}^{\prime}\right)$ and a $10_{\mathrm{H}}^{\prime}$ with specified couplings would be needed to implement natural and stable doublet-triplet splitting (see secs. 5, 7 and Ref. [25]).

It is worth noting that the spectrum of the type exhibited in Eq. (8), including the adjoint of $\mathrm{SU}(4)$-Color $(1,1,15)$ (in fact two of them) and a second $(2,2,1)_{\mathrm{H}}$ which is light (massless), is quite feasible within the D-Brane and F-theory constructions, as in Refs. [87] 
and [88] respectively 18 . It, of course, still needs to be checked whether precisely the spectrum of Eq. (8), together with the three-generation MSSM spectrum and desired gross pattern of Yukawa couplings can be realized within such constructions or variants thereof. Note also that the spectrum of Eq. (8) is devoid of $(1,1,6)$ and thereby of possibly dangerous color triplets which might induce rapid $d=5$ proton decay depending upon their Yukawa couplings. Such a spectrum in fact possesses some specially desirable features with no obvious problems, as noted below:

(a) Removal of Mismatch: The spectrum of Eq. (8) helps remove the mismatch between $M_{s t}$ and $M_{U}$ as follows. The gauge couplings $g_{2 L}, g_{2 R}$ and $g_{4}$ of the $G(2,2,4)$ symmetry, unified at the string scale $M_{\text {st }} \approx 4 \times 10^{17} \mathrm{GeV}$, would run down to lower energies with their respective $\beta$-functions. It may be verified that, in the presence of the multiplets given by $\mathrm{H}_{\text {extra }}^{\prime}$ and the MSSM-GUT spectrum, the one-loop beta functions for $g_{2 L}, g_{2 R}$ and $g_{4}$ (including the gauge, Higgs and matter contributions) are equal, with $b_{2 L}=b_{2 R}=b_{4}=6$. Since, for MSSM, the gauge coupling $\alpha_{U}$ at the unification scale $M_{U}$ is small $\approx 0.04$, we expect that two-loop corrections will not alter the running between $M_{s t}$ to $M_{U}$ significantly. Thus, the three gauge couplings of $G(2,2,4)$, unified at the string-scale, will still remain unified while running down upto the conventional MSSM GUT-scale $M_{U} \approx 2 \times 10^{16} \mathrm{GeV}$, where the symmetry $G(2,2,4)$ will break to the SM symmetry by utilizing the VEVs of $\left\{(1,2,4)_{\mathrm{H}}+\right.$ $\left.(1,2, \overline{4})_{\mathrm{H}}\right\}$ and of $(1,1,15)_{\mathrm{H}}$ listed in the subset $\mathrm{H}_{\mathrm{SSB}}$. This resolves the mismatch between the MSSM-unification at $M_{U}$ and the string-unification of $G(2,2,4)$ at $M_{\text {st }} \approx 4 \times 10^{17} \mathrm{GeV}$.

(b) Preserving the Ratio $M_{U} / M_{s t}$ : I should add that of the four possible resolutions of the mismatch between $M_{s t}$ and $M_{U}$ for a string-unified $G(2,2,4)$ symmetry, mentioned above, the fourth one, needing just two extra multiplets, beyond those needed for SSB ( see the second subset on the RHS of Eq.(8)), has a practical advantage. This is because, for the first three, one way or another, the ratio $\left(M_{U} / M_{s t}\right)$ is near unity, where as for the fourth one the conventional values of $M_{U} \approx 2 \times 10^{16} \mathrm{GeV}$ and $M_{s t} \approx 4 \times 10^{17} \mathrm{GeV}$ are preserved, and thereby their ratio of $M_{U} / M_{s t} \approx 1 / 20$ as well. This latter value of the ratio of $M_{U} / M_{s t}$ clearly goes well with the hierarchical entries that are needed for an understanding of the masses and mixings of the charged fermions ( see Sec. 5) and of the neutrinos ( see Sec. 6), that are realized through higher dimensional operators.

(c) Desired $\alpha_{3}\left(m_{Z}\right)$ : There is an additional benefit of the extra multiplets listed above. It is well known that with a "typical" SUSY spectrum at the electroweak scale, and without any GUT-scale threshold corrections, the MSSM gauge couplings unify, with 2-loop running from the weak to the GUT-scale, for $\alpha_{3}\left(m_{Z}\right) \approx 0.127$. This is somewhat higher than the observed PDG average value of $\alpha_{3}\left(m_{Z}\right)_{\text {expt }}=0.1176 \pm 0.002$ (see second reference in Ref. [81]). Now, the one-loop GUT-scale threshold corrections, including contributions from the extra multiplets shown in Eq. (8), can be calculated in terms of two parameters: (i) the ratio of the two GUT-scale VEVs $\left\langle(1,2, \overline{4})_{\mathrm{H}}\right\rangle /\left\langle(1,1,15)_{\mathrm{H}}\right\rangle$ and (ii) the ratio $M / M_{U}$, where $M$ denotes an "average" mass of the superheavy GUT-scale particles, assumed to have a common mass, for simplicity. One finds 94 that, allowing for a reasonably wide range of variation of these two ratios, the GUT-scale threshold correction to $\alpha_{3}\left(m_{Z}\right)$ ranges between - $(0.006-0.012)$,

\footnotetext{
${ }^{18}$ I thank Mirjam Cvetic, Alon Faraggi, Arthur Hebecker, George Leontaris and Stuart Raby for discussions on these points.
} 
which gives $\alpha_{3}\left(m_{Z}\right)$ within one standard deviation of the observed value. This demonstrates that with the addition of just two extra multiplets (i.e. one $(1,1,15)_{\mathrm{H}}^{\prime}$ and one $\left.(2,2,1)_{\mathrm{H}}^{\prime}\right)$ of an assumed string-origin, given by $\mathrm{H}_{\text {extra }}^{\prime}$, together with one $(1,1,15)_{\mathrm{H}}$ and the sub-sets $\mathrm{A}$ and $\mathrm{B}$ listed in $\mathrm{H}_{\mathrm{SSB}}$ that are needed to implement symmetry breaking, a string-unified $G(2,2,4)$ symmetry can be fully consistent with the observed GUT-based MSSM gauge coupling unification at $M_{U} \approx 2 \times 10^{16} \mathrm{GeV}$, with a clear benefit as regards the predicted value of $\alpha_{3}\left(m_{Z}\right)$.

(d) Achieving Doublet-Triplet Splitting and Realizing the MSSM Spectrum below the GUTScale: As noted above the spectrum of Eq.(8) is devoid of the multiplet $(1,1,6)$, while it possesses two $(1,1,15)$ 's. Without the $(1,1,6)$, the four dimensional theory is free from the color-triplets and thus does not face the familiar doublet-triplet splitting problem. The latter could pose the danger of extra rapid $d=5$ proton decay, even if the color-triplets have masses $\sim$ (one to a few $) \times \mathrm{M}_{\mathrm{GUT}}$, unless the Yukawa couplings of the $(1,1,6)$ are adequately suppressed compared to those of the light $(2,2,1)_{\mathrm{H}}$. Now, many of the string solutions ( see e.g. the works of T. Kobayashi et al. and B. Assel et al. in [86] and those in Refs. [87] and [88] ) do in fact possess the (1,1,6)-multiplet in 4D and assign heavy GUT-scale mass to it, which is allowed. However, there can also exist solutions ( see e.g. the Appendix B.2 of Ref.[87], and also Ref.[88], with the remarks there in), which are devoid of the $(1,1,6)$ of $G(2,2,4)$ and thus of the color triplets in $4 \mathrm{D}$.

I should add that the multiplet $(1,1,6)$ has in fact been utilized in these works to give GUT-scale masses to the $d^{c}$ and $\overline{d^{c}}$-like components lying within the multiplets $S_{H} \sim$ $(1,2,4)_{H}$ and $\bar{S}_{H} \sim(1,2, \overline{4})_{H}$, which are used to break the symmetry $G(2,2,4)$ to the Standard Model. This is done by using a superpotential coupling of the form

$$
W \supset\left[S_{H}^{2} \cdot(1,1,6)+\bar{S}_{H}^{2} \cdot(1,1,6)\right]
$$

and utilizing the GUT-scale VEVs of the RH sneutrino-like fields in $S_{H}$, and likewise in $\bar{S}_{H}$. This in turn leads to just the MSSM-like spectrum below the GUT-scale, as desired. The same result can, however, be achieved by utilizing the multiplet $(1,1,15)$ of Eq. (8), instead of $(1,1,6)$, through a superpotential coupling of the form

$$
W \supset S_{H} \cdot(1,1,15) \cdot \bar{S}_{H},
$$

and using the same VEVs as above. In short, the spectrum of Eq. (8), being present in $4 \mathrm{D}$, would naturally provide a resolution of the doublet-triplet splitting problem through string-theoretic compactification in higher dimensions,. Simultaneously it would lead to to just the MSSM spectrum below the GUT-scale, which goes well with the observed gauge coupling unification.

In this sense, the spectrum of Eq. (8) accompanying three chiral families and a light bi-doublet $(2,2,1)_{H}$, appears to have definite advantages. It would thus be worth checking if precisely such a spectrum, and (ambitiously) with the desired gross pattern of Yukawa couplings, and supersymmetry breaking, can be derived as an allowed solution within a suitable string-theoretic construction.

Returning to the task of a comparison between a non-GUT string-derived symmetry versus a GUT-symmetry in $4 \mathrm{D}$, we see that the mismatch between $M_{s t}$ and $M_{U}$ can thus 
quite plausibly be removed ( as in cases (i)-(iii)), or accounted for (as in case (iv)), for a nonGUT string-derived symmetry like $G(2,2,4)$. At the same time, a SUSY GUT symmetry like $\mathrm{SU}(5)$ or $\mathrm{SO}(10)$ in $4 \mathrm{D}$ would have an advantage in this regard because it is guaranteed to keep the gauge couplings together between $M_{s t}$ and $M_{U}$ (even if $M_{U} \sim M_{s t} / 20$ ), and thus not even encounter the problem of a mismatch between the two scales. A supersymmetric GUT-solution (like SU(5) or SO(10)), however, has a possible disadvantage as well, because it needs certain color triplets to become superheavy by the so-called double-triplet splitting mechanism (see Ref. [26] and references therein and discussion in Sec. 7), in order to avoid the problem of rapid proton decay. However, no such mechanism has emerged yet, in string theory, for the GUT-like solutions [89]. Four-dimensional SUSY SO(10) models possessing technically natural and stable doublet-triplet splitting have been constructed [26], and I will discuss their consequences in Sec. 7. I may add, however, that they are not so simple, and one may wonder if such a mechanism can arise from an underlying theory, like string theory.

Non-GUT string solutions, based on symmetries like $G(2,2,4)$ or $G(2,1,4)$ for example, can have a distinct advantage in this regard, in that the dangerous color triplets, which would induce rapid proton decay, can be naturally projected out for such solutions through string compactification, see e.g. 95, 96. This is also feasible within the D-brane ( see Appendix B.2 of Ref.[87]) and very likely within F-theory constructions (see remarks in Ref.[88. Furthermore, the non-GUT solutions invariably possess new "flavor" gauge symmetries, which distinguish between families. These symmetries are immensely helpful in explaining qualitatively the observed fermion mass-hierarchy (see e.g. Ref. [96]) and resolving the so-called naturalness problems of supersymmetry such as those pertaining to the issues of squarkdegeneracy [97], and quantum gravity-induced rapid proton decay [98.

Weighing the advantages and possible disadvantages of both, it seems hard at present to make a priori a clear choice between a presumed SUSY GUT (like SO(10)) versus a non-GUT (like $G(2,2,4)$ ) string-solution. As expressed elsewhere [5], it therefore seems prudent to keep both options open and pursue their phenomenological consequences. Now, the advantages of an effective $G(2,2,4)$ or $\mathrm{SO}(10)$ symmetry in understanding masses and mixings of all fermions including neutrinos turn out to be essentially identical, especially if one uses lowdimensional Higgs multiplets (as opposed to large-dimensional ones) to break $\mathrm{SO}(10)$ or $G(2,2,4)$ to the SM in 4D (See discussion in Secs. 4-6 and Ref. [25]). I will thus proceed by assuming that either a suitable $G(2,2,4)$-solution with a mechanism to resolve the mismatch between $M_{U}$ and $M_{\text {string }}$ of the sort mentioned above (most preferably the fourth one), or a realistic $\mathrm{SO}(10)$-solution with the needed doublet-triplet mechanism, will emerge from string theory. As we will see in Sec. 7, a study of proton decay can help distinguish between these two alternatives.

Before discussing in more detail the consequences of an intact SUSY SO(10) or a stringunified $G(2,2,4)$-symmetry in $4 \mathrm{D}$, I should mention briefly an interesting line of attempt which proposes to achieve unification through higher-dimensional ( $\mathrm{D}=5$ or 6 ) orbifold GUTmodels based on SUSY SU(5) [99] or SO(10) [100].

D=5 or 6 Orbifold GUT-Models: These models are motivated, on the one hand, by the successes of SUSY grand unification in 4D (as listed, for example, in (i)-(x) in Sec. 2 for the $G(2,2,4) / S O(10)$ case), as well as by its shortcomings in the Higgs-sector, such as the 
problem of doublet-triplet splitting, which is relevant to the case of an intact SUSY SU(5) or $S O(10)$ in $4 \mathrm{D}$. On the other hand, they have also been inspired by the promising structure of orbifold compactification [101] of the ten-dimensional heterotic string-theory [102], with the inclusion of background fields, such as the Wilson lines [103].

Combining some of the virtues of both sets of ideas, the higher dimensional orbifold GUTmodels 99, 100, assume that a SUSY GUT symmetry, like $S U(5)$ or $S O(10)$, is effective as a point-particle field theory in a higher-dimensional space-time $(\mathrm{D}=5$ for the $S U(5)$-case [99] and $\mathrm{D}=5$ or 6 for the $S O(10)$-case [100]). Such a symmetry is presumed to break down either to the SM or to an $U(1)_{X}$-extended SM, with broken supersymmetry, operating in $4 \mathrm{D}$ (the latter would be applicable if the parent symmetry in 6D is $S O(10)$ ), through compactification on an orbifold of the extra spatial dimension(s), subject to a choice(s) of suitable GUTsymmetry breaking boundary conditions. Assuming that such a choice(s) would be allowed by an underlying theory, these models have the merit that they can achieve: (a) gauge coupling unification at least in the leading order, (b) desired GUT-symmetry breaking, as well as (c) doublet-triplet splitting through compactification, without involving the Higgs mechanism of 4D GUTs.

Predictions on proton decay in these orbifold GUT-models, which have been studied better only for the $S U(5)$-case, vary widely as regards the lifetime and branching ratios of different decay modes, depending upon the choice of location of the multiplets and of their orbifold parities. For the $S U(5)$-case, the choice includes the possibility of separate locations of the $\overline{5}$ and 10 of a single $S U(5)$-family. These can be either on the GUT-symmetrybreaking or symmetry -preserving branes, or in the bulk (which is symmetry-preserving). As a generic feature, however, all sources of $d=4$ and $d=5$ proton decay operators (including the color-triplet Higgsino-exchange contribution) are forbidden in these models.

Now, depending upon the choice of locations and boundary conditions (including parities) as mentioned above, there are several possibilities for contributions from the effective $(X, Y)$ gauge-boson mediated $d=6$ proton decay operators, especially for the $\mathrm{D}=5 S U(5)$-case, which is better studied. These operators can lead to either (a) suppressed or even forbidden $d=6$ proton decay (see e.g. Altarelli and Feruglio in [99]); or (b) to $d=6$ proton decays with estimated lifetimes $\sim 10^{34}$ years (subject to large uncertainties), with a rather distinctive flavor structure that the branching ratios for proton decaying via $e^{+} \pi^{0}, \mu^{+} \pi^{0}, e^{+} K^{0}, \mu^{+} K^{0}$, $\bar{\nu} \pi^{+}$and $\bar{\nu} K^{+}$can all be comparable (see e.g. the third paper by Hall and Nomura in [99]); or (c) to $d=6$ proton decays with an estimated lifetime $\sim 10^{35}$ years (for a compactification scale $M_{c} \sim 10^{14} \mathrm{GeV}$ ) with the novel feature that the $\mu^{+} K^{0}$ and $\bar{\nu}_{\mu} K^{+}$decay modes of the proton are dominant (see e.g. the second paper by Hebecker and March-Russell in [99]). Clearly the flavor structures of (b) and (c) are very distinct from those of the predictions of SUSY $S U(5)$ and $S O(10)$ models in $\mathrm{D}=4$ (see Sec. 7).

Given the merits of these higher-dimensional orbifold GUT-models as noted above, and their possible distinctive signatures in proton decay (especially for the $S U(5)$-case), they seem to provide a viable path to higher unification which is worth pursuing. Noting that, as point-particle field theories in $5 \mathrm{D}$ or $6 \mathrm{D}$, they are non-renormalizable, they should be viewed as effective theories needing an ultraviolet completion. This poses the question as to whether such an effective $\mathrm{D}=5$ or $\mathrm{D}=6$ orbifold GUT-model can be derived, along with 
the desired boundary conditions, from an underlying theory, in particular the superstring theory. The task here is similar in spirit to that of deriving, for example, a string-unified $G(2,2,4)$ symmetry, with the desired Higgs multiplets in $4 \mathrm{D}$ to achieve symmetry-breaking, but without the problem of doublet-triplet splitting in 4D. As discussed in the preceding, there are promising semi-realistic string-solutions of the latter type (see e.g. [87,88] and remarks there in). Deriving such a string solution corresponding to a suitable 5D or 6D orbifold GUT-model with the desired boundary conditions remains a challenge ( A promising attempt in this regard has in fact been made by T. Kobayashi et al., referenced in [86]).

Before returning to the main aspects of my talk, I should mention that, despite their merits as mentioned above, the $\mathrm{D}=5$ orbifold $S U(5)$ models [99], and even the $\mathrm{D}=6$ orbifold $S O(10)$ models, that rely entirely on orbifold boundary conditions to break $S O(10)$ to an $U(1)_{X}$ - extended SM symmetry in 4D (see e.g. the first two papers of Ref. [100]), suffer from a drawback in that they do not seem to provide a natural understanding of the mass-scales of the observed neutrino oscillations. ( By contrast, this is readily possible within a SUSY $S O(10)$ or a suitable string-unified $G(2,2,4)$ symmetry of the type mentioned above, in $4 \mathrm{D}$ ( see Sec. 6)) To be specific, for the $\mathrm{D}=5 S U(5)$-case, the difficulty in this regard is similar to that of $S U(5)$ in $\mathrm{D}=4$. That is: (i) the three $\mathrm{RH}$ neutrinos have to be added by hand as singlets; but (ii) without the (B-L) local symmetry in $S U(5)$, there is no good control over their Majorana masses; and (iii) without the $S U(4)$-color-relation between the Dirac mass of $\nu_{\tau}$ and the mass of the top quark (see Eq. (6)), the former can vary widely. As a result, the seesaw-mass for the corresponding light neutrino, depending crucially on the last two features, becomes uncertain by more than five orders of magnitude ( see discussion in Sec. 6). Now, one might have expected that the $\mathrm{D}=6$ orbifold $S O(10)$ models to do better in this regard. But, as noted in the second paper of Ref. [100], generating suitable Majorana masses for the RH neutrinos does not seem to go well, for this case,with an understanding of the weak angle, together with the removal of anomalies of the $6 \mathrm{D}$ bulk.

There exists an alternative line of attempt based on $\mathrm{D}=5$ orbifold $S O(10)$ models ( see the last three papers in Ref. [100]), which combine orbifold boundary conditions to achieve a partial breaking of $S O(10)$, for example to the symmetry $G(2,2,4)$, together with the Higgs mechanism on the brane to break $G(2,2,4)$ to the SM symmetry in $4 \mathrm{D}$. This class of orbifold $S O(10)$ models fare better by providing at least a gross understanding of the mass- scales of neutrino oscillations, to within factors of 10 to 100, depending upon the compactification scale $\mathrm{M}_{\mathrm{c}}$ (see e.g. the fourth and fifth papers of Ref. [100]) ${ }^{19}$ Thus the idea of combining the orbifold boundary conditions with the Higgs mechanism to break $S O(10)$ in $5 \mathrm{D}$ to the SM symmetry in $4 \mathrm{D}$, via the intermediary of the $G(2,2,4)$ symmetry, seems to be promising.

For comparison, it is worth noting that this alternative approach to symmetry-breaking within 5D $S O(10)$ models, that combines the orbiflod boundary conditions with the Higgs mechanism to complete the task, is in fact quite analogous in its spirit to the attempts within string theory that aim to derive a string-unified $G(2,2,4)$ symmetry in $4 \mathrm{D}$ through compactification of the ten-dimensional string theory and break the $G(2,2,4)$ symmetry through the Higgs mechanism in $4 \mathrm{D}$ to the SM at the GUT-scale. As discussed in the beginning of this sub-section, semi-realistic string-unified $G(2,2,4)$-solutions do exist, with

\footnotetext{
${ }^{19}$ I thank Radovan Dermisek for a communication in this regard.
} 
the desired Higgs system to break the $G(2,2,4)$ symmetry in $4 \mathrm{D}$ to the SM, some of which can be free from the doublet-triplet spltting problem ( like the orbifold GUT models in $5 \mathrm{D}$ or $6 \mathrm{D})$. Such a string-unified $G(2,2,4)$-symmetry, as also a SUSY $S O(10)$ model, both in $4 \mathrm{D}$, have a clear advantage of providing a natural understanding of the mass-scales of neutrino oscillations ( see Sec. 6). Furthermore, subject to the assumption of a suitable flavor symmetry ( which may have a string-origin), they also provide a predictive framework for understanding at least the gross pattern and some intriguing features of the masses and mixings of all fermions including neutrinos ( see discussion in Sec. 5).

Returning now to the main task of my talk, I next discuss the spontaneous breaking of $\mathrm{SO}(10)$ or $G(2,2,4)$ to the $\mathrm{SM}$, which will be relevant to our understanding of the masses and mixings of all fermions including neutrinos (Secs. 5 and 6 ).

\section{Breaking $\mathbf{S O}(\mathbf{1 0})$ or $G(2,2,4)$ to the $\mathbf{S M}$}

For compactness of notation, I will present the Higgs system that would be responsible for breaking $\mathrm{SO}(10)$ to the SM. A completely analogous set of Higgs multiplets can be used to break $G(2,2,4)$ to the SM. Now two distinct sets of multiplets have been used to break $\mathrm{SO}(10)$ to the SM, each having some advantages over the other.

One of these, which I will follow in the rest of my talk, utilizes a minimal set of lowdimensional Higgs multiplets (LOH) 104,105, 106, 107, 108, 109, 110, to break a supersymmetric $\mathrm{SO}(10)$ symmetry to $\mathrm{SU}(3)^{\mathrm{c}} \times \mathrm{U}(1)_{\mathrm{cm}}$. The same set is also used to generate a predictive framework for understanding masses and mixings of all fermions including neutrinos ( see e.g. [25,113,114]). Minimally, it consists of the multiplets:

$$
\mathrm{H}_{\mathrm{LOH}}=\left\{10_{\mathrm{H}}, 16_{\mathrm{H}}, \overline{16}_{\mathrm{H}}, 45_{\mathrm{H}}, S_{\mathrm{H}}\right\}
$$

The $16_{\mathrm{H}}$ is paired with $\overline{16}_{\mathrm{H}}$ to preserve supersymmetry at the GUT-scale. Of these, the $\mathrm{VEV}$ of $\left\langle 45_{\mathrm{H}}\right\rangle \sim \mathrm{M}_{\mathrm{U}}$ in the (B-L)-direction breaks $\mathrm{SO}(10)$ to $G(2,2,1,3)$ and those of $\left\langle 16_{\mathrm{H}}\right\rangle=\left\langle\overline{16}_{\mathrm{H}}\right\rangle \sim \mathrm{M}_{\mathrm{U}}$ along the $\left\langle\tilde{\bar{\nu}}_{\mathrm{RH}}\right\rangle$ and $\left\langle\tilde{\nu}_{\mathrm{RH}}\right\rangle$-components break $G(2,2,1,3)$ to the SM $G(2,1,3)$ at the unification-scale $\mathrm{M}_{\mathrm{U}}$. Now $G(2,1,3)$ breaks at the EW scale by the VEV of $\left\langle 10_{\mathrm{H}}\right\rangle$ and that of the EW doublet of the down-type in $\left\langle 16_{\mathrm{H}}^{d}\right\rangle$ to $\mathrm{SU}(3)^{\mathrm{c}} \times \mathrm{U}(1)_{\mathrm{em}}$. The singlet $S$, with a $\mathrm{VEV} \sim \mathrm{M}_{\mathrm{U}}$, is added to serve as a flavon field carrying, in the simplest case, a gauged U(1)-flavor charge [111]. It serves to generate a hierarchical pattern of fermion masses and mixings of the type envisaged in Ref. [25]. This will be discussed briefly in the following section.

Before entering into the role of the low-dimensional Higgs system in describing masses and mixings of fermions, I should mention a notable alternative, which has been used widely in the literature (for an incomplete list of references see Ref. [112]). It utilizes a large-dimensional Higgs system (LGH) consisting of $\mathrm{SO}(10)$-tensorial multiplets like $\left\{126_{\mathrm{H}}, \overline{126}_{\mathrm{H}}, 210_{\mathrm{H}}\right.$ and possibly $120_{\mathrm{H}}$ and/or $\left.54_{\mathrm{H}}\right\}$. It is useful to note the decomposition of the $\mathrm{SO}(10)$-multiplet $126_{\mathrm{H}}=\left\{\Delta_{\mathrm{L}}=\left(3_{\mathrm{L}}, 1_{\mathrm{R}}, 10^{\mathrm{c}}\right)+\bar{\Delta}_{\mathrm{R}}=\left(1_{\mathrm{L}}, 3_{\mathrm{R}}, \overline{10}^{\mathrm{c}}\right)+\left(2_{\mathrm{L}}, 2_{\mathrm{R}}, 15^{\mathrm{c}}\right)+\left(1,1, \overline{6}^{\mathrm{c}}\right)\right\}$ under the subgroup $G(2,2,4)$. 
One advantage of $\overline{126}_{\mathrm{H}}$ is that it has a renormalizable Yukawa coupling of the form $h_{i j} 16_{i} 16_{j} \overline{126}_{\mathrm{H}}$ (and likewise also $120_{\mathrm{H}}$ for $i \neq j$ ), where $16_{i}$ (with $i=1,2,3$ ) denotes the three families. The VEV of $\left\langle\Delta_{R}\right\rangle \sim \mathrm{M}_{\mathrm{U}}$ (with $\left\langle\Delta_{\mathrm{L}}\right\rangle \approx$ "0") breaks B-L by two units and provides superheavy Majorana masses to the RH neutrinos, which are needed to implement the Type I seesaw mechanism. Such a breaking of B-L automatically preserves the familiar R-parity $=(-1)^{3(\mathrm{~B}-\mathrm{L})+2 \mathrm{~S}}$ of SUSY theories, and thereby avoids the dangerous $d=4$ protondecay operators and also yields a stable LSP to serve as cold dark matter (CDM). By contrast, $16_{\mathrm{H}}$ and $\overline{16}_{\mathrm{H}}$ of the low-dimensional Higgs system break B-L by one unit and thereby break the familiar R-parity. This difference is, however, not significant in practice, because for LOH one can still define consistently a matter-parity (under which $16_{i}$ is odd, but all other fields exhibited in Eq. (9) are even), which fully serves the desired purpose by allowing all the desired interactions but forbidding the dangerous $d=4$ proton decay operators and yielding a stable LSP to serve as CDM.

Now both the LGH [112] and the LOH systems (see e.g. Refs. [25], [111], [113] and [114]), the latter often with the assumption of a suitable flavor symmetry, have been developed to yield predictive and successful frameworks for describing masses and mixings of all fermions including neutrinos. An example of this kind (Ref. [25]) for the case of LOH system will be presented briefly in the following section.

While both the LGH and the LOH systems seem to have some merits and are worth pursuing, I will base my discussion in the following on masses and mixings of all fermions including neutrinos (Secs. 5 and 6) and on proton decay (Sec. 7) by confining to the LOH system only. Briefly, the reasons are as follows: (i) There exists, for the case of SUSY $\mathrm{SO}(10)$, a simple mechanism for a natural[104,105] and stable[26] doublet-triplet splitting in the case of $\mathrm{LOH}$, which thus avoids rapid $d=5$ proton decay without any fine tuning. It is not clear whether such a situation can emerge for LGH. (ii) The large-dimensional Higgs multiplets (like $126_{\mathrm{H}}, \overline{126}_{\mathrm{H}}, 210_{\mathrm{H}}$ and possibly $120_{\mathrm{H}}$ ) tend to give rather large and differing GUT-scale threshold corrections (exceeding even 30 to 50\%) to the three gauge couplings from the split sub-multiplets. Such corrections are more controlled for the case of LOH. Incorporating stable doublet-triplet splitting, the latter tends to provide sharper predictions for proton lifetimes including upper limits for a given SUSY spectrum[26] (see Sec. 7). (iii) The models based on $\mathrm{LOH}$ (see e.g. [25], [111]) predict that the heaviest of the three light neutrinos has a mass that is naturally of order $1 / 10 \mathrm{eV}$, their masses being hierarchical (see Sec. 6). This is in good agreement with the atmospheric neutrino oscillation data. (iv) For the LGH system with multiplets like $126_{\mathrm{H}}, \overline{126}_{\mathrm{H}}$ and others, the unified gauge coupling blows up rapidly just above the GUT-scale $M_{U}$, making the physics between string/Planck-scale and GUT scale unclear. Finally, (v) while our understanding of the non-perturbative aspects of string theory is still lacking, the weakly interacting heterotic string theory solutions do not seem to yield the large-dimensional tensorial multiplets like $126_{\mathrm{H}}$ and $120_{\mathrm{H}}$, but they do yield the low-dimensional ones as listed in Eq. (9) [115].

With these distinctions in mind, I present in the next section an attempt to understand the masses and mixings of all fermions including neutrinos within the $\mathrm{SO}(10) / G(2,2,4)$ framework by using the low-dimensional Higgs (LOH) system as in Eq. (6). 


\section{Masses and Mixings of Fermions in $\mathbf{S O}(10)$ or $G(2,2,4)$}

There are some distinct advantages of describing the masses and mixings of the three families, including the neutrinos, within a symmetry like $\mathrm{SO}(10)$. Essentially the same holds for the case of a string-unified $G(2,2,4)$-symmetry as well, especially if one confines to the low-dimensional Higgs system (LOH), as in Eq. (9). In either case, owing to quarklepton unification through $\mathrm{SU}(4)$-color and up-down correlation through $\mathrm{SU}(2)_{\mathrm{L}} \times \mathrm{SU}(2)_{\mathrm{R}}$, the four mass-matrices $\mathrm{M}_{\mathrm{U}}, \mathrm{M}_{\mathrm{D}}, \mathrm{M}_{l}$ and $\mathrm{M}_{\nu}^{\mathrm{D}}$ (the last one denotes the Dirac mass-matrix of the neutrinos) get interrelated (see e.g. Ref. [25]). This reduces the number of independent parameters significantly and thereby increases predictivity. An example of such a correlation is given by Eqs. (5) and (6), of which the first one is known to go well with observations, while the second one plays an important role in ensuring the success of the seesaw (see Sec. 6). Some of these relations, like Eq. (5), hold for SU(5) as well, but not Eq. (6).

In what follows (for reasons noted in the previous section), I will limit my discussion to using only the minimal low-dimensional Higgs system $(\mathrm{LOH})$ for generating the masses and mixings of all fermions including neutrinos (Dirac as well as Majorana) for the case of a supersymmetric $\mathrm{SO}(10)$ model. The Higgs multiplets in this case are given by the set (see Eq. (9)):

$$
\mathrm{H}_{\mathrm{LOH}}=\left\{10_{\mathrm{H}}, 16_{\mathrm{H}}, \overline{16}_{\mathrm{H}}, 45_{\mathrm{H}}, \mathrm{S}_{\mathrm{H}}\right\}
$$

An analogous Higgs system consisting of the multiplets $\left[(2,2,1)_{\mathrm{H}}\left\{(2,1,4)_{\mathrm{H}}+(1,2, \overline{4})_{\mathrm{H}}\right\},\left\{(2,1, \overline{4})_{\mathrm{H}}+\right.\right.$ $\left.\left.(1,2,4)_{\mathrm{H}}\right\},(1,1,15)_{\mathrm{H}}, \mathrm{S}_{\mathrm{H}}\right]$ can be used for the case of the supersymmetric $G(2,2,4)$ symmetry both to break $G(2,2,4)$ to $\mathrm{SU}(3)^{\mathrm{c}} \times \mathrm{U}(1)_{\mathrm{cm}}$ and to generate a desirable and identical pattern for the masses and mixings of the fermions as in the case of $\mathrm{SO}(10)$.

To illustrate the advantages alluded to above, let me present briefly the supersymmetric $\mathrm{SO}(10)$-framework developed in Ref. [25] (elaborated further in [111]) for treating the masses and mixings of the fermions within the minimal low-dimensional Higgs system (Eq. (6)) ${ }^{20}$ The question that one faces in the very beginning is this: can this minimal Higgs system provide a realistic pattern for fermion masses and mixings? Now $\mathbf{1 0}_{\mathbf{H}}$ (even several $\mathbf{1 0}$ 's) cannot provide certain desirable features - i.e. family-antisymmetry and (B-L)-dependence in the mass matrices - which are, however, needed to suppress $V_{c b}$ while enhancing $\theta_{23}^{\nu}$ on the one hand, and accounting for features such as $m_{\mu}^{0} \neq m_{s}^{0}$ on the other hand. Furthermore, a single $\mathbf{1 0}_{\mathbf{H}}$ cannot generate CKM mixings. At the same time, $\mathbf{1 0}_{\mathbf{H}}$ is the only multiplet among the ones in the minimal Higgs system (Eq. (6)) which can have cubic couplings with the matter fermions which are in the 16's. This apparent impasse disappears as soon as one allows for not only cubic but also effective non-renormalizable quartic and even higher dimensional couplings of the minimal set of Higgs fields with the fermions. Such effective couplings can of course arise quite naturally through exchanges of superheavy states (e.g. those in the string-tower or those having GUT-scale masses $\sim$ a few $\mathrm{M}_{\mathrm{GUT}}$ (say)) involving renormalizable couplings. And importantly, all such effective couplings that are allowed by

\footnotetext{
${ }^{20}$ Alternative $\mathrm{SO}(10)$-models of fermion masses and mixings based on the low-dimensional Higgs system have been developed with some overlapping features and some distinctions (see e.g. Refs. [113] and [114]).
} 
the symmetry relevant to the 'lower' energy (including $\mathrm{SO}(10)$ and possible flavor symmetries, see for example[111]) are expected to arise at least through quantum gravity effects, which would be scaled by $\mathrm{M}_{\text {string }}$ or $\mathrm{M}_{\text {planck }}$.

The $3 \times 3$ Dirac masses matrices for the four sectors $(u, d, l, \nu)$ proposed in Ref. [25] are motivated in part by the group theory of $S O(10) / G(2,2,4)$, which severely restricts the effective cubic and quartic couplings (and thus the associated mass-patterns), for the minimal Higgs system. They are also motivated in part by the notion that flavor symmetries [116] distinguishing between the three families lead to a hierarchical pattern for the mass matrices (i.e. with the element " 33 " $\gg " 23 " \gg " 22 " \gg " 12 " \gg " 11 "$ etc.), so that the lighter family gets its mass primarily through its mixing with the heavier ones. Such a hierarchical pattern can in fact be realized by introducing (for example) just a single gauged U(1)-flavor symmetry and an SO(10)-singlet flavon field S, together with an appropriate assignment of the flavor charges to all the fields [111. Subject to the constraints as mentioned above, the effective Yukawa couplings turn out to be rather unique. Confining to the relevant lowest dimensional terms, they are given by [25,111]:

$$
\begin{aligned}
\mathcal{L}_{\text {Yuk }} & =h_{33} \mathbf{1 6}_{3} \mathbf{1 6}_{3} \mathbf{1 0}_{H} \\
& +\left[h_{23} \mathbf{1 6}_{2} \mathbf{1 6}_{3} \mathbf{1 0}_{H}(S / M)+a_{23} \mathbf{1 6}_{2} \mathbf{1 6}_{3} \mathbf{1 0}_{H}\left(\mathbf{4 5}_{H} / M^{\prime}\right)(S / M)^{p}\right. \\
& \left.+g_{23} \mathbf{1 6}_{2} \mathbf{1 6}_{3} \mathbf{1 6}_{H}^{d}\left(\mathbf{1 6}_{H} / M^{\prime \prime}\right)(S / M)^{q}\right] \\
+ & {\left[h_{22} \mathbf{1 6}_{2} \mathbf{1 6}_{2} \mathbf{1 0}_{H}(S / M)^{2}+g_{22} \mathbf{1 6}_{2} \mathbf{1 6}_{2} \mathbf{1 6}_{H}^{d}\left(\mathbf{1 6}_{H} / M^{\prime \prime}\right)(S / M)^{q+1}\right] } \\
+ & {\left[g_{12} \mathbf{1 6}_{1} \mathbf{1 6}_{2} \mathbf{1 6}_{H}^{d}\left(\mathbf{1 6}_{H} / M^{\prime \prime}\right)(S / M)^{q+2}+a_{12} \mathbf{1 6}_{1} \mathbf{1 6}_{2} \mathbf{1 0}_{H}\left(\mathbf{4 5}_{H} / M^{\prime}\right)(S / M)^{p+2}\right] }
\end{aligned}
$$

Typically we expect $M^{\prime}, M^{\prime \prime}$ and $M$ to be of order $M_{\text {string }}$ [117].The VEV's of $\left\langle\mathbf{4} 5_{H}\right\rangle$ (along B-L), $\left\langle\mathbf{1 6}_{H}\right\rangle=\left\langle\overline{\mathbf{1 6}}_{H}\right\rangle$ (along standard model singlet sneutrino-like component) and of the $\mathrm{SO}(10)$-singlet $\langle S\rangle$ are of the GUT-scale, while those of $\mathbf{1 0}_{H}$ and of the down type $\mathrm{SU}(2)_{L}$-doublet component in $\mathbf{1 6}_{H}$ (denoted by $\mathbf{1 6}_{H}^{d}$ ) are of the electroweak scale [25, 118]. Depending upon whether $M^{\prime}\left(M^{\prime \prime}\right) \sim M_{\mathrm{GUT}}$ or $M_{\text {string }}$ (see comment in Ref. [117]), the exponent $p(q)$ is either one or zero [119].

With the effective Yukawa couplings given in Eq. (10), the Dirac mass matrices of quarks and leptons of the three families, at the unification scale, take the form (Ref. [25]). ${ }^{21}$

$$
\begin{aligned}
& M_{u}=\left[\begin{array}{ccc}
0 & \epsilon^{\prime} & 0 \\
-\epsilon^{\prime} & 0 & \sigma+\epsilon \\
0 & \sigma-\epsilon & 1
\end{array}\right] \mathcal{M}_{u}^{0} ; \quad M_{d}=\left[\begin{array}{ccc}
0 & \eta^{\prime}+\epsilon^{\prime} & 0 \\
\eta^{\prime}-\epsilon^{\prime} & 0 & \eta+\epsilon \\
0 & \eta-\epsilon & 1
\end{array}\right] \mathcal{M}_{d}^{0} \\
& M_{\nu}^{D}=\left[\begin{array}{ccc}
0 & -3 \epsilon^{\prime} & 0 \\
-3 \epsilon^{\prime} & 0 & \sigma-3 \epsilon \\
0 & \sigma+3 \epsilon & 1
\end{array}\right] \mathcal{M}_{u}^{0} ; \quad M_{l}=\left[\begin{array}{ccc}
0 & \eta^{\prime}-3 \epsilon^{\prime} & 0 \\
\eta^{\prime}+3 \epsilon^{\prime} & 0 & \eta-3 \epsilon \\
0 & \eta+3 \epsilon & 1
\end{array}\right] \mathcal{M}_{d}^{0}
\end{aligned}
$$

\footnotetext{
${ }^{21}$ The zeros in " $11 "$, " $13 "$, " $31 "$, and " $22 "$ elements signify that they are relatively small. For instance, the " 22 " -elements are set to zero because (restricted by flavor symmetries, see below), they are meant to be less than ("23")("32")/ "33" 10 -2 , and thus unimportant for our purposes. Likewise, for the other "zeros."
} 
These matrices are defined in the gauge basis and are multiplied by $\bar{\Psi}_{L}$ on left and $\Psi_{R}$ on right. For instance, the row and column indices of $M_{u}$ are given by $\left(\bar{u}_{L}, \bar{c}_{L}, \bar{t}_{L}\right)$ and $\left(u_{R}, c_{R}, t_{R}\right)$ respectively. Note the group-theoretic up-down and quark-lepton correlations: the same $\sigma$ occurs in $M_{u}$ and $M_{\nu}^{D}$, and the same $\eta$ occurs in $M_{d}$ and $M_{l}$. It will become clear that the $\epsilon$ and $\epsilon^{\prime}$ entries, arising through the coupling of $\left(10_{\mathrm{H}} \cdot 45_{\mathrm{H}}\right)$ in the $a_{23}$ and $a_{12}$-terms, are proportional to B-L and are antisymmetric in the family space (as shown above). Thus, the same $\epsilon$ and $\epsilon^{\prime}$ occur in both $\left(M_{u}\right.$ and $\left.M_{d}\right)$ and also in $\left(M_{\nu}^{D}\right.$ and $\left.M_{l}\right)$, but $\epsilon \rightarrow-3 \epsilon$ and $\epsilon^{\prime} \rightarrow-3 \epsilon^{\prime}$ as $q \rightarrow l$. Such correlations result in an enormous reduction of parameters and thus in increased predictivity. Furthermore, it is precisely because of this family-antisymmetric (B-L)-dependent contribution that one obtains a group theoretic understanding of why $V_{\mathrm{cb}}$ is small while $\theta_{23}^{\nu}$ is large. Although the entries $\sigma, \eta, \epsilon, \eta^{\prime}$, and $\epsilon^{\prime}$ will be treated as parameters, consistent with assignment of flavor-symmetry charges (see below), we would expect them to be hierarchical with $(\sigma, \eta, \epsilon) \sim 1 / 10$ and $\left(\eta^{\prime}, \epsilon^{\prime}\right) \sim 10^{-3}-10^{-4}$ (say).

The entries 1 and $\sigma$ arise respectively from $h_{33}$ and $h_{23}$ couplings, while $\hat{\eta} \equiv \eta-\sigma$ and $\eta^{\prime}$ arise respectively from $g_{23}$ and $g_{12}$-couplings. As mentioned above, the (B-L)-dependent antisymmetric entries $\epsilon$ and $\epsilon^{\prime}$ arise respectively from the $a_{23}$ and $a_{12}$ couplings. This is because, with $\left\langle\mathbf{4 5} \mathbf{5}_{H}\right\rangle \propto \mathrm{B}$-L, the product $\mathbf{1 0} \mathbf{0}_{H} \times \mathbf{4 5 _ { H }}$ contributes as a $\mathbf{1 2 0}$, whose coupling is family-antisymmetric. Thus, for the minimal Higgs system (see Eq. (6)), (B-L)-dependence can enter only through family off-diagonal couplings of $\mathbf{1 0} \mathbf{H} \cdot \mathbf{4 5} \mathbf{H}$ as in $a_{23}$ and $a_{12}$-terms. Thus, for such a system, the diagonal "33" entries are necessarily (B-L)-independent (as shown in Eq. (8)). This in turn makes the relations like $m_{b}\left(M_{X}\right) \approx m_{\tau}$ (barring corrections of order $\epsilon^{2}$, which turn out to have the right sign and magnitude (see Ref. [25]) robust. This feature would, however, be absent if one had used $\overline{\mathbf{1 2 6}}_{\mathbf{H}}$, whose coupling is family-symmetric and can give (B-L) dependent contributions to the " 33 "-elements.

As alluded to above, such a hierarchical form of the mass-matrices, with $h_{33}$-term being dominant, is attributed in part to flavor gauge symmetry(ies) that distinguishes between the three families, [111 and in part to higher dimensional operators involving for example $\left\langle\mathbf{4 5}_{H}\right\rangle / M^{\prime}$ or $\left\langle\mathbf{1 6}_{H}\right\rangle / M^{\prime \prime}$, which are supressed by $M_{\mathrm{GUT}} / M_{\text {string }} \sim 1 / 10$, if $M^{\prime}$ and/or $M^{\prime \prime} \sim$ $M_{\text {string. }}$.

To discuss the neutrino sector one must specify the Majorana mass-matrix of the $\mathrm{RH}$ neutrinos as well. As in the case of effective Yukawa couplings giving Dirac masses, this would arise through effective couplings of the form[121]:22

$$
\mathcal{L}_{\text {Maj }}=f_{i j} \mathbf{1 6}_{i} \mathbf{1 6}_{j} \overline{\mathbf{1 6}}_{H} \overline{\mathbf{1 6}}_{H} / M
$$

where the $f_{i j}$ 's include appropriate powers of $\langle S\rangle / M$, in accord with flavor symmetry. For the $f_{33}$-term to be leading, being $\sim 1$, we must assign the flavor-charge of $\overline{16}_{\mathrm{H}}$ to be $-a$ (see

\footnotetext{
${ }^{22}$ There is an impression, sometimes conveyed in the literature, that a multiplet like $\overline{126}_{\mathrm{H}}$ of $\mathrm{SO}(10)$ (or equivalently $\left(1,3_{\mathrm{R}}, 10\right)_{\mathrm{H}}$ of $\left.G(2,2,4)\right)$ is needed to give Majorana masses to the $\mathrm{RH}$ neutrinos. While $\overline{126}_{\mathrm{H}}$ would contribute through renormalizable Yukawa couplings, as explained before for the case of the lowdimensional Higgs multiplets exhibited in Eq. (9), the effective non-renormalizable interactions, allowed by all relevant symmetries, should be expected to arise through exchange of heavy states in the string-tower (see Ref. [121), and at least through quantum gravity. Thus $\overline{126}_{\mathrm{H}}$ is not really needed for the purpose. The virtue of Eq. (9) in understanding the mass-scale of atmospheric neutrino oscillation, owing to the suppression of the operator by a factor $\sim\left(M_{\mathrm{GUT}}^{2} / M_{\mathrm{st}}\right)$ is discussed in Sec. 6 .
} 
Ref. [120]). This leads to a hierarchical form for the Majorana mass-matrix [25]:

$$
M_{R}^{\nu}=\left[\begin{array}{lll}
x & 0 & z \\
0 & 0 & y \\
z & y & 1
\end{array}\right] M_{R}
$$

Following the flavor-charge assignments given in Ref. [120], we expect $|y| \sim\langle S / M\rangle \sim 1 / 10$, $|z| \sim(\langle S / M\rangle)^{2} \sim 10^{-2}(1$ to $1 / 2),|x| \sim(\langle S / M\rangle)^{4} \sim\left(10^{-4}-10^{-5}\right)$ (say). The "22" element (not shown) is $\sim(\langle S / M\rangle)^{2}$ and its magnitude is taken to be $<\left|y^{2} / 3\right|$, while the "12" element (not shown) is $\sim(\langle S / M\rangle)^{3}$.

As it turns out, the mass-scale of the heaviest of the three light neutrinos $\left(m\left(\nu_{3}\right)\right)$ that would emerge from Eqs. (11) and (13) and would correspond (because of the hierarchical pattern of the masses) to the mass-scale of the atmospheric neutrino oscillation, would have an important implication for physics at truly high energies. I would therefore discuss a derivation of this mass-scale and its implications separately in the next section. But for now, it would be interesting to note the prediction of the simple patterns of the mass-matrices given in Eqs. (11) and (13) for the gross features of the masses and mixings of quarks and leptons including the neutrinos, by stating some features of the neutrino-sector in advance of Sec. 6 .

To make predictions (postdictions), one needs to determine the parameters appearing in Eqs. (11) and (13). For the purposes of this talk, I will ignore possible phases in these parameters and thus CP violations, as was done in Ref. [25]. The patterns of mass-matrices suggested in Ref. [25] (i.e. in Eqs. (11) and (13)) has, however, been studied subsequently by allowing for phases in the parameters (which could arise from complex effective couplings and/or VEVs) in Ref. 122. It was found (rather remarkably) in this work that the observed $\mathrm{CP}$ violation of the CKM form with the desired Wolfenstein paramters can be realized within the pattern of mass-matrices of Ref. [25], while preserving its successes.

Turning to the case of real parameters for simplicity, thereby ignoring $\mathrm{CP}$ violation, the parameters $\left(\sigma, \eta, \epsilon, \epsilon^{\prime}, \eta^{\prime}, \mathcal{M}_{u}^{0}, \mathcal{M}_{D}^{0}\right.$, and $\left.y\right)$ can be determined by using, for example, $m_{t}^{\text {phys }}=174 \mathrm{GeV}, m_{c}\left(m_{c}\right)=1.37 \mathrm{GeV}, m_{S}(1 \mathrm{GeV})=110-116 \mathrm{MeV}, m_{u}(1 \mathrm{GeV})=6 \mathrm{MeV}$, the observed masses of $e, \mu$, and $\tau$ and $m\left(\nu_{2}\right) / m\left(\nu_{3}\right) \approx(1 / 6)$, as inputs. One is thus led, for this CP conserving case, to the following fit for the parameters, and the associated predictions. 25] [In this fit, we leave the small quantities $x$ and $z$ in $M_{R}^{\nu}$ undetermined and proceed by assuming that they have the magnitudes suggested by flavor symmetries (i.e., $x \sim\left(10^{-4}-10^{-5}\right)$ and $z \sim 10^{-2}(1$ to $1 / 2)$ (see remarks below Eq. (13))]:

$$
\begin{gathered}
\sigma \approx 0.110, \quad \eta \approx 0.151, \quad \epsilon \approx-0.095, \quad\left|\eta^{\prime}\right| \approx 4.4 \times 10^{-3}, \\
\epsilon^{\prime} \approx 2 \times 10^{-4}, \quad \mathcal{M}_{u}^{0} \approx m_{t}\left(M_{U}\right) \approx 120 \mathrm{GeV}, \\
\mathcal{M}_{D}^{0} \approx m_{b}\left(M_{U}\right) \approx 1.5 \mathrm{GeV}, \quad y \approx-(1 / 18) .
\end{gathered}
$$

The hierarchical entries in the Dirac and Majorana mass-matrices necessarily predict a hierarchical pattern with normal hierarchy for the seesaw-generated masses of the light neutrinos (i.e. $m\left(\nu_{3}\right) \gg m\left(\nu_{2}\right)>m\left(\nu_{1}\right)$ ). Taking this into account, the mass-matrices given 
by Eqs. (11) and (13) lead to the following predictions for the masses and mixings of the quarks and some of the light neutrinos [25]:

$$
\begin{aligned}
& m_{b}^{\circ}\left(M_{U}\right) \approx m_{\tau}^{\circ}\left(1-8 \epsilon^{2}\right) \Rightarrow m_{b}\left(m_{b}\right) \approx(4.7-4.9) \mathrm{GeV}, \\
& \sqrt{\Delta m_{\mathrm{atm}}^{2}} \equiv \sqrt{\Delta m_{31}^{2}} \approx m\left(\nu_{3}\right) \approx(1 / 24 \mathrm{eV})(1 / 2-2), \text { (see Sec. 6) } \\
& \left.V_{c b} \approx\left|\sqrt{\frac{m_{s}}{m_{b}}}\right| \frac{\eta+\epsilon}{\eta-\epsilon}\right|^{1 / 2}-\sqrt{\frac{m_{c}}{m_{t}}}\left|\frac{\sigma+\epsilon}{\sigma-\epsilon}\right|^{1 / 2} \mid \approx 0.044, \\
& \left\{\begin{array}{l}
\left.\theta_{23}^{\nu} \approx\left|\sqrt{\frac{m_{\mu}}{m_{\tau}}}\right| \frac{\eta-3 \epsilon}{\eta+3 \epsilon}\right|^{1 / 2}+\sqrt{\frac{m_{\nu_{2}}}{m_{\nu_{3}}}}|\approx| 0.437+0.408 \mid, \\
\text { Thus [123], } \sin ^{2} 2 \theta_{23}^{\nu} \approx 0.994, \quad\left(\text { for } m\left(\nu_{2}\right) / m\left(\nu_{3}\right) \approx 1 / 6\right),
\end{array}\right. \\
& V_{u s} \approx\left|\sqrt{\frac{m_{d}}{m_{s}}}-\sqrt{\frac{m_{u}}{m_{c}}}\right| \approx 0.20, \\
& \left|\frac{V_{u b}}{V_{c b}}\right| \approx \sqrt{\frac{m_{u}}{m_{c}}} \approx 0.07 \text {, } \\
& m_{d}(1 \mathrm{GeV}) \approx 8 \mathrm{MeV} \text {. }
\end{aligned}
$$

Before discussing the empirical successes of the predictions listed above, let me first comment on some aspects of the neutrino-sector pertaining to the first family. As regards $\nu_{e}-\nu_{\mu}$ and $\nu_{e}-\nu_{\tau}$ oscillations, the standard seesaw-contributions, based on the hierarchical massmatrices $M_{\nu}^{D}$ (Eq. (8)) and $M_{R}^{\nu}$ (Eq. (13)), typically lead to rather small oscillation angles $(\lesssim 0.1)$ [25]. It has however, been noted [111] that non-seesaw contribution, e.g. to $\nu_{\mathrm{L}}^{e} \nu_{\mathrm{L}}^{\mu}$ mixing mass-term $\sim(2-6) \times 10^{-3} \mathrm{eV}$, can quite plausibly arise through higher dimensional operators in accord with flavor symmetry (see Ref. [111] and [124]). Such a contribution, combined with the standard seesaw contributions based an $M_{\nu}^{D}$ and $M_{\mathrm{R}}^{\nu}$, that yields a diagonal $\left(\nu_{\mathrm{L}}^{\mu} \nu_{\mathrm{L}}^{\mu}\right)$ mass $\sim(3-10) \times^{-3} \mathrm{eV}$, can plausibly lead to $\theta_{12}^{\nu}$ as large as $\sim\left(30^{\circ}-35^{\circ}\right)$, in accord with the LMA MSW solution of the solar neutrino problem (For a review of current experimental status, see Refs. [125] and [126]). Similarly, one can obtain through non-seesaw contribution $\theta_{13}^{\nu} \approx\left(2^{\circ}-10^{\circ}\right)$, to be compared with the observed value of $\approx 9^{\circ}$ [125],126]. Thus, including the seesaw (from $M_{\nu}^{D}$ and $M_{\mathrm{R}}^{\nu}$ ) and non-seesaw contributions, together with a natural value of $y \approx-1 / 18$ (which is expected to be of order $1 / 10$ by flavor symmetry[120]), one gets:

$$
\begin{aligned}
m\left(\nu_{2}\right) & \approx(8.5) \times 10^{-3} \mathrm{eV} \text { (seesaw) } \\
m\left(\nu_{1}\right) & \approx(1-\text { few }) \times 10^{-3} \mathrm{eV} ; \text { thus } \Delta m_{21}^{2} \approx 7 \times 10^{-5} \mathrm{eV}^{2} \\
\theta_{12}^{\nu} & \approx\left(20^{\circ}-35^{\circ}\right)(\text { non-seesaw }) \\
\theta_{13}^{\nu} & \approx\left(2^{\circ}-10^{\circ}\right)(\text { non-seesaw })
\end{aligned}
$$

Here, $m\left(\nu_{2}\right)$ should be regarded as a reasonable expectation within the model, within a factor of 2 or so either way, corresponding to a natural choice of $|y| \approx 1 / 10-1 / 25$ (say). Unlike the results listed in Eq. (15), which are compelling predictions (postdictions) of the model, however, those on $\theta_{12}^{\nu}$ and $\theta_{13}^{\nu}$ noted above should be considered only as plausible and consistent possibilities within the model. 
The Majorana masses of the $\mathrm{RH}$ neutrinos $\left(N_{i} \equiv \nu_{\mathrm{R}}^{i}\right)$ are given by (see discussion in Sec. 6).

$$
\begin{aligned}
& M_{3} \approx M_{R} \approx 10^{15} \mathrm{GeV}(1 / 2-2) \\
& M_{2} \approx\left|y^{2}\right| M_{3} \approx\left(3 \times 10^{12} \mathrm{GeV}\right)(1 / 2-1) \\
& M_{1} \approx\left|x-z^{2}\right| M_{3} \sim(1 / 4-2) 10^{-5} M_{3} \sim 10^{10} \mathrm{GeV}(1 / 10-2)
\end{aligned}
$$

where $y \approx-1 / 18$ and $x \sim z^{2} \sim 10^{-4}(1 / 2-2)$ have been used in accord with flavor symmetry. [120] Note that we necessarily have a hierarchical spectrum for the light as well as the heavy neutrinos with normal hierarchy $m_{1}<m_{2} \ll m_{3}$ and $M_{1} \ll M_{2} \ll M_{3}$. Leaving out the oscillation angles $\theta_{12}^{\nu}$ and $\theta_{13}^{\nu}$, it seems remarkable that the first six predictions in Eq. (15) agree with observations, pretty well. Particularly intriguing is the (B-L)-dependent group-theoretic correlation between the contribution from the first term in $V_{c b}$ and that in $\theta_{23}^{\nu}$, which explains simultaneously why one is small $\left(V_{c b}\right)$ and the other is large $\left(\theta_{23}^{\nu}\right)$.

Another interesting point of the hierarchical BPW model[25] is that with $|y|$ being hierarchical (of order $1 / 10$ as opposed to being of order 1) and $m\left(\nu_{2}\right) / m\left(\nu_{3}\right)$ being of order $1 / 5-1 / 10$, the mixing angle from the neutrino sector $\sqrt{m\left(\nu_{2}\right) / m\left(\nu_{3}\right)}$ necessarily adds (rather than subtracts) to the contribution from the charged lepton sector (see Eq. (15)), as shown in Ref. [25]. As a result, in the BPW model, both charged lepton and neutrino-sectors give medium-large contribution $(\approx 0.4)$ which add to naturally yield a maximal $\theta_{23}^{\nu}$. At the same time, analogous entries for $V_{\mathrm{cb}}$ subtract, leading to its smallness (see Eq. (15)). This thus becomes a simple and compelling prediction of the model, based essentially on the group theory of the minimal Higgs system in the context of SO(10) or G(224) and the hierarchical nature of the mass-matrices 23

The success of the model as regards especially the first six predictions in Eq. (15) provides some confidence in the gross pattern of the Dirac and Majorana mass matrices presented above. I now proceed to discuss primarily the implications of the mass-scale of the atmospheric neutrino oscillation in the next section.

\section{Neutrino Masses Shedding Light on Unification and Our Origin}

Since the discoveries (confirmations) of the atmospheric [12] and solar neutrino oscillations, [13] at SuperKamiokande and SNO respectively, the neutrinos have emerged as being the most effective probes into the nature of higher unification. To add to these, a set of ingenius experiments have come into play to study accelerator and reactor neutrinos involving disappearance and appearance phenomena, including those at KamLAND, K2K, MINOS, OPERA, Double Chooz, T2K, Daya Bay, RENO and Nova, giving new insights, sometimes with higher precision and/or reconfirmations (For a review and notations, see e.g.

\footnotetext{
${ }^{23}$ The explanation of the largeness of $\theta_{23}^{\nu}$ together with the smallness of $V_{\mathrm{cb}}$ outlined above, based on medium-large contributions from the charged lepton and neutrino sectors, is quite distinct from alternative explanations. In paricular, in the lop-sided Albright-Barr model[113, the largeness of $\theta_{23}^{\nu}$ arises almost entirely from the lop-sidedness of the charged lepton mass matrix. This distinction between the BPW and the $\mathrm{AB}$ models leads to markedly different predictions for the rate of $\mu \rightarrow e \gamma$ decay in the two models [128.
} 
Refs. [125] and [126] and references there in. These together have led to a measurement of the two independent (mass) ${ }^{2}$-differences of: $\left|\Delta m_{32}^{2}\right| \cong\left|\Delta m_{31}^{2}\right| \equiv \Delta m_{\text {Atm }}^{2} \approx(1 / 20 \mathrm{eV})^{2}$, and $\Delta m_{21}^{2} \equiv \Delta m_{\text {solar }}^{2} \cong(1 / 115 \mathrm{eV})^{2}$, and the three oscillation angles of: $\theta_{23} \approx \pi / 4$ (within a few degrees either way), $\theta_{12} \approx \pi / 5.4$, and $\theta_{13} \approx \pi / 20$. Supplementing these measurements, cosmological studies by PLANCK now provide an upper limt on the sum of the three neutrino masses to be about $0.23 \mathrm{eV}$ [127].

In attempting to understand the mass-scales of the neutrino oscillations, one finds that, although almost the feeblest of all entities of nature, simply by virtue of their tiny masses, the neutrinos seem to possess a subtle clue to some of the deepest laws of nature pertaining to the unification-scale as well as the nature of the unification symmetry. In this sense, the neutrinos provide us with a rare window to view physics at truly short distances which turn out to be as short as nearly $10^{-30} \mathrm{~cm}$. Furthermore it appears most likely that the origin of their tiny masses may be at the root of the origin of matter-antimatter a symmetry in the early universe [15] and thereby at the root of our own origin.

Before discussing an understanding of the neutrino masses in more detail, let me explain qualitatively why the neutrinos are able to provide us with the window to view physics at truly short distances. The reason is two-fold. First, it is because of the seesaw mechanism[14] (I will confine to Type I seesaw only), that combines the heavy (or superheavy $\gg 1 \mathrm{TeV}$ ) Majorana mass $M_{R}^{\nu}$ of the RH neutrino with the familiar Dirac mass of the neutrino $\left(m_{D}^{\nu} \lesssim\right.$ $100 \mathrm{GeV})$, to yield a light LH neutrino with a mass $m\left(\nu_{L}\right) \cong\left(m_{D}^{\nu}\right)^{2} / M_{R}^{\nu} \ll m_{D}^{\nu}$. Thus, the seesaw mechanism plays a crucial role by providing us with a natural reason for the neutrinos to be light or even superlight, as specified in the following.

But, contrary to to the impression often found in the literature, the seesaw mechanism, by itself, is not sufficient to provide even an estimate having any degree of certainty as regards how light or how heavy can the neutrinos be, while still remaining much lighter than (say) 10-100 GeV. This is because, without the constraints of an appropriate unification-symmetry and the unification-scale, it has no clue to the magnitude of either the Majorana mass $M_{R}^{\nu}$ (which, in general, can vary from the string or Planck scale $\sim 10^{18} \mathrm{GeV}$ to (say) a few $\mathrm{TeV}$ ), or the Dirac mass $m_{D}^{\nu}$ (which, for any given species, unconstrained by symmetry, could lie anywhere in the range of $100 \mathrm{GeV}$ to (say) $1 \mathrm{MeV}$ ). Hence the uncertainty in the estimate of the seesaw-mass which can exceed even twenty orders of magnitude.

But, as I will discuss below, if the seesaw mechanism arises in the context of an appropriate unification symmetry possessing $\mathrm{SU}(4)$-color, as in $\mathrm{SO}(10)$ or a string-unified $G(2,2,4)$ symmetry, and is tied to the unification-scale $\left(M_{U} \sim 2 \times 10^{16} \mathrm{GeV}\right)$, such that the latter is essentially the (B-L)-breaking scale, both the Majorana mass $M_{R}^{\nu}$ and the Dirac mass $m_{D}^{\nu}$, suitably generalized to $3 \times 3$-matrices for the three-family case, get constrained. In this case, as explained below, one quite naturally obtains the desired magnitudes for the mass-scales of the atmospheric and solar neutrino oscillations. It is thus the combination of the three ingredients: (a) the seesaw mechanism, (b) a suitable unification-symmetry, and (c) the unification-scale $\sim 2 \times 10^{16} \mathrm{GeV}$ that is needed to provide an understanding of the neutrino mass-scales. This, in turn, is the reason why the neutrinos turn out to be so revealing about: (i) the nature of the unification-symmetry, (ii) the scale of unification (in particular that of (B-L)-breaking), and (iii) the nature of the neutrino mass (being Majorana not Dirac), 
all of which pertain to physics at truly high energies $\left(\sim 10^{16} \mathrm{GeV}\right)$ and thus short distances $\sim 10^{-30} \mathrm{~cm}$.

I now turn to discuss these aspects in more detail by exhibiting the steps that lead to the mass-scale $\left(m\left(\nu_{3}\right)\right)$ of the heaviest of the three light neutrinos. This, as we will see, will represent the mass-scale of the atmospheric neutrino oscillation as well, as quoted in Eq. (12). I will follow the framework discussed in Sec. 5, which is based on the symmetry $\mathrm{SO}(10)$ or a string-unified $G(2,2,4)$ symmetry, combined with an assumed U(1)-flavor symmetry [120] that serves to provide the desired inter-family mass-hierarchy. For reasons discussed in Sec. 4, the low-dimensional Higgs system of Eq. (9) is used to break the symmetry SO(10) to the low-energy symmetry $\mathrm{SU}(3)^{\mathrm{c}} \times \mathrm{U}(1)_{\mathrm{em}}$. (An analogous system applies for the symmetry $G(2,2,4))$. The resulting hierarchical pattern of masses and mixings of all fermions including those given by the Dirac and Majorana mass-matrices of the neutrinos are exhibited in Eqs. (11) and (13), which have their origins in the effective couplings of Eqs. (10) and (12) respectively.

To obtain an estimate of the mass-scale of the heaviest of the three light neutrinos, it is useful to consider first only the third family, ignoring the first two families for a moment, although the final answer will be obtained by considering the full three-family system, including mixings among the families. 25] Using the effective couplings given by Eq. (12), we expect the heaviest Majorana mass $M_{3} \approx M_{\mathrm{R}}$ of Eq. (13) to be given by:

$$
M_{\mathrm{R}}=\frac{f_{33}\left\langle\overline{16}_{\mathrm{H}}\right\rangle^{2}}{M} \approx\left(10^{15} \mathrm{GeV}\right)(1 / 2-2)
$$

where I have put $\left\langle\overline{16}_{\mathrm{H}}\right\rangle \approx M_{U} \approx 2 \times 10^{16} \mathrm{GeV}, M \approx M_{\text {string }} \approx 4 \times 10^{17} \mathrm{GeV}[90]$, and the flavorsymmetry-allowed leading Majorana coupling $f_{33} \approx 1$, in line with the value of the leading Dirac Yukawa coupling $h_{33} \approx h_{\text {top }}$. An uncertainty factor of $(1 / 2-2)$ is allowed around a centrally expected value of $M_{\mathrm{R}}$ of about $10^{15} \mathrm{GeV}$. Using the seesaw formula, in the absence of the first two families, one then obtains the light $\mathrm{LH} \nu_{\mathrm{L}}^{\tau}$ with a mass $m\left(\nu_{\mathrm{L}}^{\tau}\right) \approx m\left(\nu^{\tau}\right)_{\operatorname{Dirac} / \mathrm{M}_{\mathrm{R}}}^{2}$. Now, allowing for mixings among the three families as given in Eqs. (11) and (13) (with hierarchical entries, only the 2-3 mixing turns out to be important for the purpose), the heaviest seesaw-generated mass is given by[25]:

$$
m\left(\nu_{3}\right) \approx B \frac{m\left(\nu_{\text {Dirac }}^{\tau}\right)^{2}}{M_{R}}
$$

The quantity $B$ represents the effect of 2-3 family-mixing (which, as mentioned above, is the dominant effect, representing mixing) and is given by $B=(\sigma+3 \epsilon)(\sigma+3 \epsilon-2 y) / y^{2}$ (see Eq. (24) of Ref. [25]). Recall that the same mixing successfully explains the near maximality of $\sin ^{2} 2 \theta_{23}^{\nu} \approx 0.994$ (see Eq. (15) and discussion in Sec. 5). Thus $B$ is fully calculable within the model since the parameters $\sigma, \eta, \epsilon$, and $y$ are determined in terms of inputs involving some quark and lepton masses (as noted in Eq. (14)). In this way, one obtains $B \approx(2.9 \pm 0.5)$. The Dirac mass of the tau-neutrino is obtained by using the $S U(4)$-color relation (see Eq. (6)): $m\left(\nu_{\text {Dirac }}^{\tau}\right) \approx m_{\text {top }}\left(M_{U}\right) \approx 120 \mathrm{GeV}$. The mass of the heaviest of the three light neutrinos, including mixing, is thus given by:

$$
m\left(\nu_{3}\right) \approx \frac{(2.9)(120 \mathrm{GeV})^{2}}{10^{15} \mathrm{GeV}}(1 / 2-2)
$$


Noting that for hierarchical entries - i.e. for $(\sigma, \epsilon$, and $y) \sim 1 / 10$ - one naturally obtains a hierarchical spectrum of neutrino-masses: $m\left(\nu_{1}\right) \lesssim m\left(\nu_{2}\right) \sim(1 / 10) m\left(\nu_{3}\right)$, we thus get:

$$
\sqrt{\Delta m_{\mathrm{atm}}^{2}} \equiv \sqrt{\left|\Delta m_{31}^{2}\right|} \approx m\left(\nu_{3}\right) \approx(1 / 24 \mathrm{eV})(1 / 2-2)
$$

This agrees remarkably well with the SuperK value of $\left(\sqrt{\Delta m_{\text {Atm }}^{2}}\right)_{\mathrm{SK}}(\approx 1 / 20 \mathrm{eV})$. As mentioned in the introduction, the success of this prediction provides clear support for (i) the existence of $\nu_{R}$, (ii) the notion of $S U(4)$-color symmetry that gives $\nu_{\mathrm{R}},(\mathrm{B}-\mathrm{L})$ and $m\left(\nu_{\text {Dirac }}^{\tau}\right)$, (iii) the SUSY unification-scale, with the assumption of a single-step breaking of $\mathrm{SO}(10)$ to the SM, that gives $M_{\mathrm{B}-\mathrm{L}} \approx M_{U}$ and there by $M_{R}$, and, of course, (iv) the seesaw mechanism.

We note that alternative symmetries such as $S U(5)$ would have no compelling reason to introduce the $\nu_{R}$ 's. Even if one did introduce $\nu_{R}^{i}$ by hand, there would be no symmetry to relate the Dirac mass of $\nu_{\tau}$ to the top quark mass. Thus $m\left(\nu_{\text {Dirac }}^{\tau}\right)$ would be an arbitrary parameter in $S U(5)$, which, could well vary from say $1 \mathrm{GeV}$ to $100 \mathrm{GeV}$. Furthermore, without B-L as a local symmetry, the Majorana masses of the RH neutrinos, which are singlets of $S U(5)$, can well be as high as the string scale $\sim 4 \times 10^{17} \mathrm{GeV}$ (say), and as low as say $1 \mathrm{TeV}$. Thus, as mentioned above, within $S U(5)$, the absolute scale of the mass of $\nu_{3}$, obtained via the familiar seesaw mechanism[14, would be uncertain by some twenty orders of magnitude.

Other effective symmetries such as $[S U(3)]^{3}\left[17\right.$ and $S U(2)_{L} \times S U(2)_{R} \times U(1)_{B-L} \times S U(3)^{C}$ (see Refs. [9], [59]) would give $\nu_{R}$ and B-L as a local symmetry, but not the desired $S U(4)$ color mass-relations: $m\left(\nu_{\text {Dirac }}^{\tau}\right) \approx m_{t}\left(M_{X}\right)$ and $m_{b}\left(M_{X}\right) \approx m_{\tau}$ (see Eqs. (5) and (6)). Flipped $S U(5) \times U(1)[129$ on the other hand would yield the desired features for the neutrinosystem, but not the empirically favored $b$ - $\tau$ mass relation (Eq. (4a)). Thus, combined with the observed $b / \tau$ mass-ratio, the SuperK data on atmospheric neutrino oscillation seems to clearly select out the effective symmetry in $4 \mathrm{D}$ being either string-unified $G(2,2,4)$ or $S O(10)$, as opposed to the other alternatives mentioned above. It is in this sense that the neutrinos, by virtue of their tiny masses, provide crucial information on the unification-scale as well as on the nature of the unification-symmetry in $4 D$, as alluded to in the introduction.

It is not just the mass-scale of the atmospheric neutrino oscillation that receives an explanation within the $G(2,2,4) / S O(10)$-framework of Sec. 5 . Within the same framework, and with a natural choice of the parameter $|y| \approx 1 / 10-1 / 20$, motivated by the flavor symmetry (see Eq. (10) and discussion in Sec. 5), one also obtains the desired magnitude for the mass-scale of solar neutrino oscillation - i.e.

$$
\sqrt{\Delta m_{\text {solar }}^{2}}=\sqrt{\Delta m_{21}^{2}} \approx m\left(\nu_{2}\right) \sim(1 / 10) \sqrt{\left|\Delta m_{\text {atm }}^{2}\right|}
$$

, as observed [125]126]. In short, it seems quite a feat that the four ingredients (i)-(iv) listed below Eq.(21), acting together, combining the interplay of physics at two vastly different scales - $M_{U} \approx 2 \times 10^{16} \mathrm{GeV}$ and $M_{\mathrm{EW}} \sim 100 \mathrm{GeV}$ - end up in yielding both the atmospheric and the solar neutrino oscillation mass-scales with the right magnitudes (within factors of $2-10)$ ! 


\subsection{Neutrinos at the root of our Origin?}

I will now discuss briefly that the tiny neutrino masses not only shed light on the nature of the unification symmetry and the unification scale, but they may well be at the root of our own origin. This comes about because of a combination of two reasons [15]: (a) The heavy Majorana masses of the RH neutrinos (Eq. (17)), which enter into an understanding of the tiny neutrino masses via the seesaw mechanism, necessarily violate lepton number and $B-L$ by two units $(|\Delta L|=|\Delta(B-L)|=2)$, and (b) the discovery of non-perturbative electroweak sphaleron effects [16], which violate $B+L$ but conserve $B-L$, and remain in thermal equilibrium in the temperature range of about $10^{12} \mathrm{GeV}$ to $200 \mathrm{GeV}$.

As a result, they efficiently erase any pre-existing baryon/lepton asymmetry that satisfies $\Delta(\mathrm{B}+\mathrm{L}) \neq 0$, but $\Delta(\mathrm{B}-\mathrm{L})=0$. This is one reason why standard GUT-baryogenesis satisfying $\Delta(\mathrm{B}-\mathrm{L})=0$ (as in minimal $\mathrm{SU}(5)$ ) (however small) becomes irrelevant to the observed baryon asymmetry of the universe. On the other hand, purely electroweak baryogenesis based on the sphaleron effects - although a priori an interesting possibility appears to be excluded for the case of the standard model without supersymmetry, and highly constrained as regards the available parameter space for the case of the supersymmetric standard model, owing to the mass of the Higgs boson being rather high $(\approx 125 \mathrm{GeV})$. As a result, in the presence of electroweak sphalerons [16], the mechanism of baryogenesis via leptogenesis [15] has emerged as perhaps the most attractive and promising mechanism to generate the observed baryon asymmetry of the universe.

Intriguingly, this mechanism directly relates our understanding of the light neutrino masses to our own origin. The question of whether this mechanism can quantitatively explain the magnitude of the observed baryon-asymmetry depends however crucially on the Dirac as well as the Majorana mass-matrices of the neutrinos, including the phases and the eigenvalues of the latter-i.e. $M_{1}, M_{2}$ and $M_{3}$ (see Eq. (14)).

This issue has been discussed in many excellent reviews [130]. Here, to be concrete, I will present briefly the work of Ref. [131], which is carried out in the context of a realistic and predictive framework for fermion masses and neutrino oscillations [25], based on the symmetry $G(2,2,4)$ or $S O(10)$, as discussed in Sec. 5, with $\mathrm{CP}$ violation treated as in Ref. [122]. I will primarily quote the results and refer the reader to Ref. [131] for more details, especially for the discussion on inflation and relevant references.

The basic picture is this. Following inflation, the lightest $\mathrm{RH}$ neutrinos $\left(N_{1}\right.$ 's) with a mass $\approx 10^{10} \mathrm{GeV}(1 / 4-3)$ (see Eq. (14)) are produced either from the thermal bath following reheating $\left(T_{R H} \approx\right.$ few $\left.\times 10^{9} \mathrm{GeV}\right)$, or non-thermally directly from the decay of the inflaton ${ }^{24}$ (with $T_{R H}$ in this case being about $10^{7} \mathrm{GeV}$ ). In either case, the RH neutrinos having Majorana masses decay (out of equilibrium) by utilizing their Dirac Yukawa couplings into both $l+H$ and $\bar{l}+\bar{H}$ (and corresponding SUSY modes), thus violating B-L. In the presence of $\mathrm{C}$ and $\mathrm{CP}$ violating phases, these decays produce a net lepton-asymmetry $Y_{L}=$

\footnotetext{
${ }^{24}$ In this case the inflaton can naturally be composed of the Higgs-like objects having the quantum numbers of the RH sneutrinos $\left(\tilde{\nu}_{R H}\right.$ and $\left.\overline{\tilde{\nu}}_{R H}\right)$ lying in $(1,2,4)_{H}$ and $(1,2, \overline{4})_{H}$ for $G(2,2,4)$ (or $16_{H}$ and $\overline{16}_{H}$ for $S O(10)$ ), whose VEV's break B-L and give Majorana masses to the RH neutrinos via the coupling shown in Eq. (12).
} 
$\left(n_{L}-n_{\bar{L}}\right) / s$ which is converted to a baryon-asymmetry $Y_{B}=\left(n_{B}-n_{\bar{B}}\right) / s=C Y_{L}(C \approx-1 / 3$ for MSSM) by the EW sphaleron effects. Using the Dirac and the Majorana mass-matrices of Sec. 5, with the introduction of CP-violating phases as in Ref. [122], the lepton-asymmetry produced per $N_{1}$ (or $\left(\tilde{N}_{1}+\bar{N}_{1}\right)$-pair) decay is found to be[131]:

$$
\begin{aligned}
\epsilon_{1} & \approx \frac{1}{8 \pi}\left(\frac{\mathcal{M}_{u}^{0}}{v}\right)^{2}|(\sigma+3 \epsilon)-y|^{2} \sin \left(2 \phi_{21}\right) \times(-3)\left(\frac{M_{1}}{M_{2}}\right) \\
& \approx-\left(2.0 \times 10^{-6}\right) \sin \left(2 \phi_{21}\right)
\end{aligned}
$$

Here $\phi_{21}$ denotes an effective phase depending upon phases in the Dirac as well as Majorana mass-matrices (see Ref. [131]). Note that the parameters $\sigma, \epsilon, y$ and $\left(\mathcal{M}_{u}^{0} / v\right)$ are already determined within our framework (to within 10\%) from considerations of fermion masses and neutrino oscillations [25] (see Sec. 5), including CP violation [122]. Furthermore, for concreteness (for the present case of thermal leptogenesis), we have put $M_{1} \approx 4 \times 10^{9} \mathrm{GeV}$ and $M_{2} \approx 2 \times 10^{12} \mathrm{GeV}$, in accord with Eq. (14). In short, leaving aside the phase factor $\phi_{21}$, the RHS of Eq. (19) is pretty well determined within our framework (to within about a factor of 5-10), as opposed to being uncertain by orders of magnitude either way. This is the advantage of our obtaining the lepton-asymmetry in conjunction with a predictive framework for fermion masses and neutrino oscillations. Now the phase angle $\phi_{21}$ is uncertain because we do not have any constraint yet on the phases in the Majorana sector $\left(M_{R}^{\nu}\right)$. At the same time, since the phases in the Dirac sector are relatively large (see Sec. 5 and Ref. [122]), barring unnatural cancellation between the Dirac and Majorana phases, we would naturally expect $\sin \left(2 \phi_{21}\right)$ to be sizable-i.e. of order $1 / 10$ to 1 (say).

The lepton-asymmetry is given by $Y_{L}=\kappa\left(\epsilon_{1} / g^{*}\right)$, where $\kappa$ denotes an efficiency factor representing wash-out effects and $g^{*}$ denotes the light degrees of freedom $\left(g^{*} \approx 228\right.$ for MSSM). For the model being considered [25], using discussions on $\kappa$ from Ref. [132], we obtain: $\kappa \approx(1 / 18-1 / 60)$, for the thermal case, depending upon the " $31^{\prime \prime}$ entries in the neutrino-Dirac and Majorana mass-matrices (see Refs. [25], [122]). Thus, for the thermal case, we obtain:

$$
\left(Y_{B}\right)_{\text {thermal }} / \sin \left(2 \phi_{21}\right) \approx(10-30) \times 10^{-11}
$$

In this case, for $M_{1} \approx 4 \times 10^{9} \mathrm{GeV}$, the reheat temperature would have to be about few $\times 10^{9} \mathrm{GeV}$ so that $N_{1}$ 's can be produced thermally. We see that the derived values of $Y_{B}$ can in fact account for the observed value, based on the latest PLANCK measurements, of $Y_{B} \approx(8.65 \pm 0.10) \times 10^{-11}\left[133\right.$, for a natural value of the phase angle $\sin \left(2 \phi_{21}\right) \approx(1 / 3-1)$. This case seems, however, to be in conflict with the familiar gravitino-constraint, with the gravitinos being unstable against decay to lighter MSSM-LSP particles [132 134]135]. The constraint may, however, be avoided if the gravitino is somehow heavier than about 10 $\mathrm{TeV}$, or if it is the LSP being as light as about $1 \mathrm{keV}$ [136]. An alternative and attractive possibility is the the case of non-thermal leptogenesis discussed below. This case typically needs a significantly lower reheat temperature, in accord with the gravitino constraint (noted above), and it can allow lower phase angles as well, compared to the thermal case.

For the non-thermal case, to be specific, I will assume an effective superpotential suggested in [137]: $W_{\text {eff }}^{\text {infl }}=\lambda \hat{S}\left(\bar{\Phi} \Phi-M^{2}\right)+$ (non-ren.terms) so as to implement hybrid infla- 
tion 25 Here $\hat{S}$ is a singlet field[140] and $\Phi$ and $\bar{\Phi}$ are Higgs fields transforming as $(1,2,4)$ and $(1,2, \overline{4})$ of $G(2,2,4)$ which break $G(2,2,4)$ to the SM and thereby B-L at the GUT scale and give Majorana masses to the $\mathrm{RH}$ neutrinos. Following the discussion in, [131]137] one obtains: $m_{\text {infl }}=\sqrt{2} \lambda M$, where $M=<(1,2,4)_{H}>\approx 2 \times 10^{16} \mathrm{GeV} ; T_{R H} \approx(1 / 7)\left(\Gamma_{\text {infl }} M_{P l}\right)^{1 / 2} \approx$ $(1 / 7)\left(M_{1} / M\right)\left(m_{\text {infl }} M_{P l} / 8 \pi\right)^{1 / 2}$ and $Y_{B} \approx-(1 / 2)\left(T_{R H} / m_{\text {infl }}\right) \varepsilon_{1}$. Taking the coupling $\lambda$ in a plausible range $\left(10^{-5}-10^{-6}\right)$, we get $m_{\text {infl }} \approx 3 \times\left(10^{10}-10^{11} \mathrm{Gev}\right)$, and a desired, reheat temperature (see below). Taking $M_{1} \approx(4 / 3-2 / 3) \times 10^{10} \mathrm{GeV}$ and $M_{2} \approx 2 \times 10^{12} \mathrm{GeV}$, in accord with Eq. (14), the inflaton would decay into a pair of $\mathrm{N}_{1}$ 's utilizing the coupling of Eq. (12), which in turn decay both into $l+H$ and $\bar{l}+\bar{H}$, as noted above, causing lepton asymmetry. Taking the asymmetry parameter as in Eq. (23), one quite plausibly obtains

$$
\left(Y_{B}\right)_{\text {Non-Thermal }} \approx(8-9) \times 10^{-11}
$$

in full accord with the PLANCK data, for natural values of the phase angle $\sin \left(2 \phi_{21}\right) \approx$ $(1 / 3-1 / 6)$, and with $T_{R H}$ being as low as $10^{7} \mathrm{GeV}(2-1 / 2)$. Such low values of the reheat temperature are consistent with the gravitino-constraint for $m_{3 / 2} \approx 400 \mathrm{GeV}-1 \mathrm{TeV}$ (say), even if one allows for possible decays of the gravitinos for example via $\gamma \tilde{\gamma}$-modes.

In summary, I have presented two alternative scenarios (thermal as well as non-thermal) for inflation and leptogenesis. We see that the $G(2,2,4) / S O(10)$-framework provides a simple and unified description of not only fermion masses, and neutrino oscillations, but also of baryogenesis via leptogenesis, the latter being in accord with all constraints for the nonthermal case. Each of the following features: (a) the existence of the RH neutrinos, (b) B-L local symmetry, (c) $S U(4)$-color, (d) the SUSY unification scale, (e) the seesaw mechanism, and (f) the pattern of $G(2,2,4) / S O(10)$ mass-matrices based on the minimal low-dimensional Higgs system (see Sec. 4), have played crucial roles in realizing this unified and successful description. I now turn to discuss the most intriguing consequence of grand unification.

\section{Proton Decay: The Hallmark of Grand Unification}

\section{$7.1 \quad$ Preliminaries}

Perhaps the most dramatic prediction of grand unification is proton decay. This topic has been discussed in the context of the SUSY SO $(10) / \mathrm{G}(2,2,4)$-framework, presented in Secs 4 and 5, in some detail in the review articles of Refs. [111] and [145] which are updates of the results obtained in Ref. [25]. For a concise review of works by several authors on proton decay in SUSY GUTs, see e.g. Ref. [75]. Here I will recall the older works and present briefly the more recent works [26]27] which provide a sharpening of the theoretical expectations for proton-decay lifetimes within a well-motivated class of supersymmetric $\mathrm{SO}(10)$ models by doing two things: (i) realizing a natural and stable doublet-triplet splitting (to be explained below); and (ii) including the GUT-scale threshold corrections to the running of the gauge

\footnotetext{
${ }^{25}$ For alternative attempts in inflationary leptogenesis, based on subcritical hybrid inflation, within the $G(2,2,4)$ symmetry, see Ref.[138], and for models of so-called "semi-shifted" hybrid inflation, also based on the symmetry $G(2,2,4)$, which possess certain desirable features, see[139].
} 


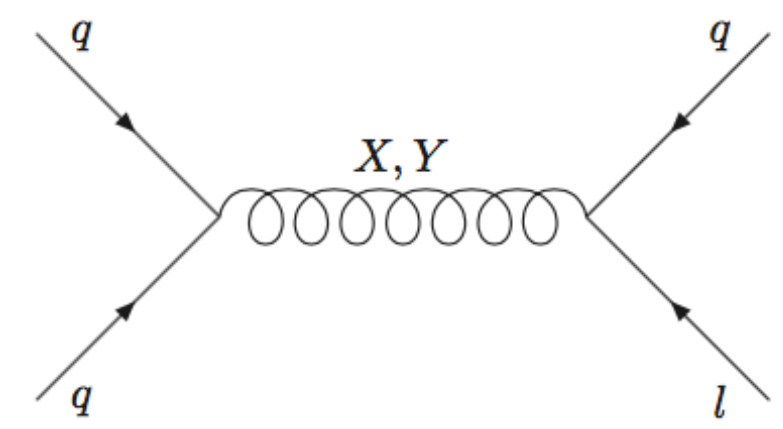

Figure 2: $d=6$ proton decay operator

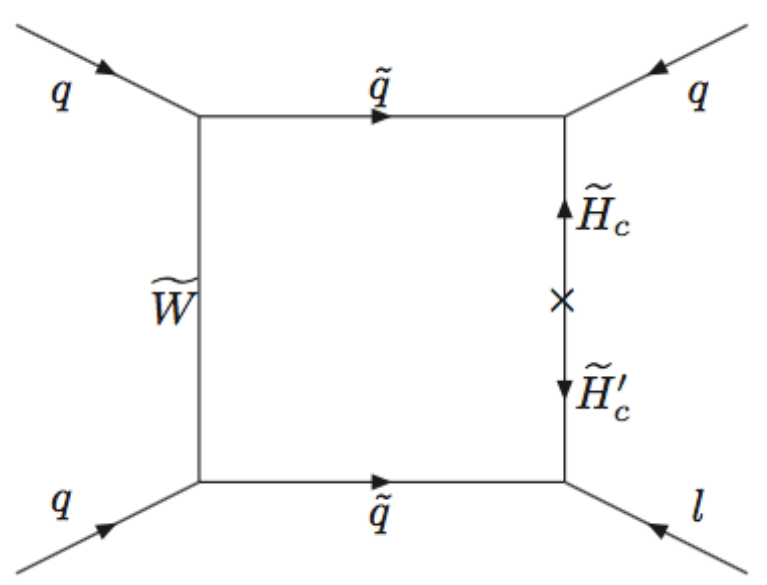

Figure 3: The standard $d=5$ proton decay operator. The $\widetilde{H}_{c}\left(\widetilde{H}_{c}^{\prime}\right)$ are color triplet(anti-triplet) Higgsinos belonging to $5_{H}\left(\overline{5}_{H}\right)$ of $S U(5)$ or $10_{H}$ of $S O(10)$.

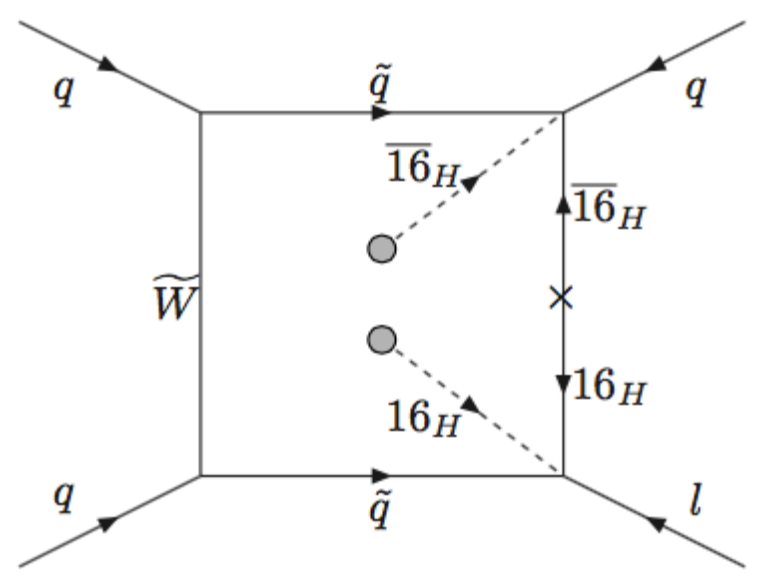

Figure 4: The "new" $d=5$ operators related to the Majorana masses of the RH neutrinos. Note that the vertex at the upper right utilizes the coupling in Eq. (9) which assigns Majorana masses to $\nu_{R}$ 's, while the lower right vertex utilizes the $g_{i j}$ couplings in Eq. (7) which are needed to generate CKM mixings. 
couplings. It turns out that these two steps, carried out in the context of a minimal set of low-dimensional Higgs multiplets, lead to an intriguing correlation equation that inversely relates the $d=6\left(p \rightarrow e^{+} \pi^{\circ}\right)$ and $d=5\left(p \rightarrow \bar{\nu} K^{+}\right)$decay amplitudes [26]. Together with the empirical lower limits on the inverse rates for these two decay modes, the correlation equation allows one to derive constrained upper limits for the same, for any given choice of the SUSY spectrum, which is updated in light of the LHC searches [27]. The discussion to follow will also include comments on the importance of the contributions to the $d=5\left(p \rightarrow \bar{\nu} K^{+}\right)$decay amplitude from a new class of diagrams 24 directly related to an understanding of the tiny neutrino masses, which are invariably ignored in the literature.

To provide a background for this discussion, in SUSY grand unification, there exist three distinct mechanisms for proton decay, exhibited in Figs. 2-4.

(i) The familiar $\mathbf{d}=\mathbf{6}$ operators mediated by $X$ and $Y$ gauge bosons of $\mathrm{SO}(10)$ (Fig. 2) (similarly for $\mathrm{SU}(5)$ as well) which yield $p \rightarrow e^{+} \pi^{\circ}$ as a dominant decay mode, with comparable $p \rightarrow \bar{\nu} \pi^{+}$mode. Generalizing the result for the minimal SUSY SU(5)-case derived in Ref. [141], to the case of $\mathrm{SO}(10)$, one obtains:

$$
\begin{aligned}
& \Gamma_{d=6}^{-1}\left(p \rightarrow e^{+} \pi^{\circ}\right) \simeq\left(1.30 \times 10^{35} \mathrm{yrs}\right)\left(\frac{0.012 \mathrm{GeV}^{3}}{\left|\alpha_{H}\right|}\right)^{2}\left(\frac{2.5}{A_{R}}\right)^{2} \\
& \quad \times\left(\frac{5.12}{f(p)}\right)\left(\frac{1 / 25}{\alpha_{X}}\right)^{2}\left(M_{X} / 10^{16} \mathrm{GeV}\right)^{4} \\
& \Gamma\left(p-\bar{\nu} \pi^{+}\right) / \Gamma\left(p \rightarrow e^{+} \pi^{\circ}\right) \approx 2[(f(p)-4) / f(p)]
\end{aligned}
$$

Here $M_{X}$ is the mass of the $X, Y$ gauge bosons which mediate proton decay; $\alpha_{X}=$ $g_{X}^{2} / 4 \pi$ denotes the $(X, Y)$-boson coupling at $M_{X} ;\left|\alpha_{H}\right| \simeq 0.012 \mathrm{GeV}^{3}$ is the relevant proton decay matrix element; $A_{R} \simeq 2.5$ is the net renormalization of the $d=6$ proton decay operator; the function $f(p)=4+\left(1+1 /\left(1+p^{2}\right)\right)^{2}$ varies between 8 and 5 as the parameter $p \equiv 2<16_{\mathrm{H}}>/<45_{\mathrm{H}}>$ varies from 0 to $\infty$, where $f(p)=5$, obtained in the limit $p \rightarrow \infty$, corresponds to the SU(5)-case. The result quoted in Eq. (26) has assumed a value for the relevant chiral lagrangian parameter $D+F \simeq 1.27$. Varying $p$, one obtains: $\Gamma\left(p \rightarrow e^{+} \pi^{\circ}\right) / \Gamma\left(p \rightarrow \bar{\nu} \pi^{+}\right) \simeq(1,1.4,2.5)$ for $(p \lesssim 1 / 3, p \approx 1, p \gg 1)$. Thus, if this branching ratio is found to be significantly lower than 2.5 , that would be strongly suggestive of $\mathrm{SO}(10$ ) (as opposed to $\mathrm{SU}(5)$ ).

While the $d=6$ inverse decay rate quoted above is largely independent of the details of the Yukawa couplings and the SUSY spectrum, it depends sensitively on the value of $M_{X}$. Often in the literature, a value of $M_{X}=M_{U} \approx 2 \times 10^{16} \mathrm{GeV}$ is used to obtain an estimate of this inverse decay rate. Although, we expect $M_{X}$ to be of order $M_{U}$, there is no reason to expect $M_{X}=M_{U}$. If one allows an uncertainty in $M_{X}$ by a factor of 3 (say) around $M_{X}=M_{U}$ either way, one would obtain: $\Gamma^{-1}\left(p \rightarrow e^{+} \pi^{\circ}\right)^{\text {estimated }} \sim\left(10^{33}-\right.$ $10^{37}$ ) yrs, having a large uncertainty by four orders of magnitude. Naively, however, we would expect $M_{X}$ and the masses of other GUT-scale split multiplets to be somewhat below $M_{U}$, so that they can be neglected in the running of the gauge couplings to achieve unification at $M_{U}$. We will see that our considerations of GUT-scale threshold 
corrections would lead to an upper limit on $M_{X}$, and thereby on $\Gamma^{-1}\left(p \rightarrow e^{+} \pi^{\circ}\right)[26$, in accord with the naive expectations.

(ii) The "Standard" $\mathbf{d}=\mathbf{5}$ operators 142 (Fig. 3) of the form $Q_{i} Q_{j} Q_{k} Q_{l} / M$ in the superpotential, which arise through exchanges of color triplet Higgsinos, which are the GUT-partners of the standard Higgs(ino) doublets in the $5+\overline{5}$ of SU(5) or 10 of $\mathrm{SO}(10)$. Thus in SUSY grand unification based on symmetries like $\mathrm{SU}(5)$ or $\mathrm{SO}(10)$, it is crucial, for consistency with the empirical lower limit on proton life time, that a suitable doublet-triplet splitting mechanism should exist that assigns GUT-scale masses to the color triplets in the $10_{H}$ of $\mathrm{SO}(10)$, or in the $5_{H}+\overline{5}_{H}$ of $S U(5)$, while keeping their electroweak doublet partners light.

Now for minimal SUSY SU(5), without large-dimensional Higgs multiplets, such a splitting can only be achieved only by extreme fine-tuning. As alluded to before in Sec. 4, for SUSY SO(10), on the other hand, there exists a natural mechanism of grouptheoretic origin 104 105] involving only low-dimensional Higgs multiplets that achieves such a splitting without any fine-tuning. The mechanism involves the introduction of a $10^{\prime}$, in addition to the minimal set given by Eq. (9), where $10^{\prime}$ is assumed to have an effective coupling of the form $10_{H} \cdot 45_{H} \cdot 10^{\prime}$, and $45_{H}$ aquires a VEV (consistent with minimization of the potential) along the B-L direction of the form: $\left\langle 45_{H}\right\rangle=$ $i, \sigma_{2} \otimes \operatorname{Diag}(a, a, a, 0,0)$. The $10^{\prime}$ does not have a VEV and does not couple to the matter multiplets $16_{i}$. It has a mass that is suppressed by 3 to 4 orders of magnitude compared to the GUT-scale owing to flavor symmetries (the same symmetries serve to stabilize the doublet-triplet (DT) splitting against all higher order operators, see Ref. [26] for details of this discussion). It can be seen that with the coupling of $10^{\prime}$ and the VEV of $\left\langle 45_{H}\right\rangle$ as above, the color triplets in the $10_{H}$ acquire GUT-scale masses, while the EW doublets remain massless in the SUSY limit. In short, the DT splitting is realized in this case of SUSY $\mathrm{SO}(10)$ without any fine-tuning.

Now, owing to (a) Bose symmetry of the superfields in $Q Q Q L / M$, (b) color antisymmetry, and especially (c) the hierarchical Yukawa couplings of the Higgs doublets, it turns out that these standard $d=5$ operators lead to dominant $\bar{\nu} K^{+}$and comparable $\bar{\nu} \pi^{+}$modes, but in all cases to highly suppressed $e^{+} \pi^{0}, e^{+} K^{0}$ and even $\mu^{+} K^{0}$ modes. For instance, for minimal SUSY SU(5), one obtains (with $\tan \beta \leq 20$, say):

$$
\left[\Gamma\left(\mu^{+} K^{0}\right) / \Gamma\left(\bar{\nu} K^{+}\right)\right]_{s t d}^{S U(5)} \sim\left[m_{u} / m_{c} \sin ^{2} \theta\right] R \approx 10^{-3}
$$

where $R \approx 0.1$ is the ratio of the relevant $\mid$ matrix element $\left.\right|^{2} \times($ phase space $)$, for the two modes.

It is clear from Fig. 3 that, following loop-integration, the $d=5$ proton-decay amplitude will be characterized by (for $m_{\widetilde{W}} \ll m_{\tilde{q}}$ ):

$$
A_{d=5}\left(p \rightarrow \bar{\nu} K^{+}\right)_{\text {std }} \infty \alpha_{2}\left(h h^{\prime} / M\right)\left(m_{\widetilde{W}} / m_{\tilde{q}}^{2}\right)
$$

where $h$ and $h^{\prime}$ are the Yukawa couplings that enter at the top and bottom corners on the right side of the loop in Fig. 3 . 
It can be seen that for minimal SUSY SU(5) (following DT splitting through finetuning $)$, the $(d=5)$-amplitude is scaled by $M=M_{H_{c}} \sim M_{G U T}$, where $M_{H_{c}}$ denotes the physical mass of the color triplets. It turns out that, in this case, gauge coupling unification requires that the color triplets be lighter than $M_{\mathrm{GUT}}$. Thus, $M$ (minimal SUSY SU(5)) $=M_{H_{c}} \lesssim M_{U}$. Despite the smallness of the relevant Yukawa couplings (including CKM mixings) entering into Fig. 3, with the amplitude being suppressed by only one power of a super heavy mass $M \lesssim M_{U}$ (Eq. (24)), the minimal SUSY SU(5) model seems to be in conflict[143] with the current SuperK limit on $\Gamma^{-1}\left(p \rightarrow \bar{\nu} K^{+}\right)$, at least with the superparticle masses being lighter than about $3-4 \mathrm{TeV}$. (see, however, comments in Ref. [144]).

For SUSY SO(10), with DT splitting achieved naturally through the coupling $10_{H} \cdot$ $45_{H} \cdot 10^{\prime}$, the situation is different. Here, one would need the insertion of the mass of $10^{\prime}$ in the right leg of Fig. 3. Thus $\mathrm{M}$ is given by an effective mass (see Refs. [25] and [26]):

$$
M(S O(10))=M_{\mathrm{eff}} \approx M_{\mathrm{GUT}}^{2} / M_{10^{\prime}}
$$

Since the mass of $10^{\prime}$ is suppressed compared to the GUT-scale owing to flavor symmetries [25,26], we expect $M=M_{\text {eff }}$ to be as high as $\sim 10^{19}-10^{20} \mathrm{GeV}$. This in turn would provide a significant suppression for the $d=5\left(p \rightarrow \bar{\nu} K^{+}\right)$-amplitude in SUSY SO(10). Together with such a suppression, $\mathrm{SO}(10)$, however, possesses an enhancement of the same amplitude (relative to SUSY SU(5)) owing in part to constraint from the nature of Yukawa coupling in $\mathrm{SO}(10)$ (see discussion in the Appendix of Ref. [25]). It turns out that, combining the suppression with the enhancement, SUSY SO(10) predicts $d=5\left(p \rightarrow \bar{\nu} K^{+}\right)$decay with inverse rates that are fully consistent with the current superK limits, but lie in an interesting range which can be probed by experiments in the near future. I will turn to this in light of recent work shortly. First, I will discuss a third mechanism, having some special features, which arises naturally within the SUSY $\mathrm{SO}(10) / G(2,2,4)$-framework and can induce $d=5$ proton decay.

(iii) The so called "new" $\mathbf{d = 5}$ operators 24,25]145] (see Fig. 4) which can generically arise through the exchange of color-triplet Higgsinos in the Higgs multiplets like $\left(16_{H}+\right.$ $\overline{16}_{H}$ ) of $S O(10)$, which have been used in an essential manner to give masses and mixings to the fermions including the $\mathrm{RH}$ neutrinos, and to break $\mathrm{SO}(10)$ (see below). Such exchanges are possible by utilizing the joint effects of (a) the couplings given in Eq. (12) which assign superheavy Majorana masses to the RH neutrinos through the VEV of $\overline{16}_{H}$, (b) the coupling of the form $g_{i j} 16_{i} 16_{j} 16_{H} 16_{H} / M$ (see Eq. (10)) which are needed, at least for the minimal Higgs-system, to generate CKM-mixings, and (c) the mass-term $M_{16} 16_{\mathrm{H}} \cdot \overline{16}_{\mathrm{H}}$ [146]. These operators also lead to $\bar{\nu} K^{+}$and $\bar{\nu} \pi^{+}$as being among the dominant modes, together, quite possibly, with the $\mu+\mathrm{K}^{\circ}$ mode (see remarks below), and they can plausibly lead to lifetimes in the range of $10^{32}-10^{35}$ yrs [see below]. These operators, though most natural in a theory with Majorana masses for the RH neutrinos, especially in the context of a low-dimensional Higgs system ( see Eq. (9)), have, however, been invariably omitted in the literature.

One distinguishing feature of the new $d=5$ operator is that they directly link proton 
decay to neutrino masses via the mechanism for generating Majorana masses of the $\mathrm{RH}$ neutrinos. The other, and perhaps most important, is that these new $d=5$ operators can induce proton decay even when the $d=6$ and standard $d=5$ operators mentioned above are absent. This is what would happen if the string theory or a higher dimensional GUTtheory would lead to an effective $G(2,2,4)$-symmetry in 4D (along the lines discussed in Sec. 3), which would be devoid of both $X$ and $Y$ gauge bosons and the dangerous colortriplets in the $10_{H}$ of $S O(10)$. By the same token, for an effective $G(2,2,4)$-theory, these new $d=5$ operators become the sole and viable source of proton decay leading to lifetimes in an interesting range (see below). And this happens primarily because the $\mathrm{RH}$ neutrinos have a Majorana mass!

In evaluating the contributions of the new $d=5$ operators to proton decay, allowance needs to be made for the fact that for the $f_{i j}$ couplings (see Eq. (12)), there are two possible $S O(10)$-contractions (leading to a $\mathbf{4 5}$ or a $\mathbf{1}$ ) of the pair $\mathbf{1 6} \overline{\mathbf{1 6}}_{H}$, both of which contribute to the Majorana masses of the $\nu_{R} \mathrm{~s}$, but only the contraction via the $\mathbf{4 5}$ contributes to proton decay. In the presence of non-perturbative quantum gravity one would in general expect both contractions to be present having comparable strengths. For example, the couplings of the 45s lying in the string-tower or possibly below the string scale, and likewise of the singlets to the $\mathbf{1 6}_{i} \overline{\mathbf{1 6}}_{H}$ pair would respectively generate the two contractions. Allowing for a difference between the relevant projection factors for $\nu_{R}$-masses versus proton decay operator, we set $\left(f_{i j}\right)_{p} \equiv\left(f_{i j}\right)_{\nu} K$, where $\left(f_{i j}\right)_{\nu}$ defined in Sec. 5 directly yields $\nu_{R}$-masses and $K$ is a relative factor of order unity [147]. As a plausible range, we take $K \approx 1 / 5-2$ (say), where $K=1 / 5$ seems to be a conservative value on the low side that would correspond to proton lifetimes near the upper end.

The results of Ref. [25] (see Eqs. (41) and (45) of this reference) giving the contributions of the new $d=5$ operators to the $p \rightarrow \bar{\nu} K^{+}$decay needs to be updated in two respects: (i) by including the projection factor $K$ mentioned above, and (ii) by taking the constraints of the LHC searches on SUSY particles [148,149] into account. One possible scenario incorporating these constraints, while preserving reasonable degree of SUSY naturalness, will be considered shortly in the course of discussing an update of Ref. [26].

For concreteness, it assumes an inverted sfermion mass-hierarchy, along the lines considered within the GUT-framework in Ref. [150], with a light stop $(\sim(500-1000) \mathrm{GeV})$ [151,152], lighter neutalino (possibly close to stop-mass), heavy first two generations $(\sim(15-20) \mathrm{TeV})$, $m_{\widetilde{W}} \sim(800-1200) \mathrm{GeV}$ and $m_{\widetilde{g}} \sim(2.5-3.5) \mathrm{TeV}$. Using Eqs. (41) and (45) of Ref. [25], we find that for a SUSY-spectrum as above, and in the absence of the standard $d=5$ operators discussed above (so that there is no interference between them), the new $d=5$ operators by themselves lead to:

$$
\Gamma^{-1}\left(p \rightarrow \bar{\nu}_{\tau} K^{+}\right)_{\text {new } d=5} \approx\left[\left(5 \times 10^{31}\right)-10^{35}\right] \text { Yrs }
$$

Here, $K=(1-1 / 5)$ has been used. Such an inverse rate is in fact quite comparable to the kind of lifetimes that would be expected for the $p \rightarrow \bar{\nu} K^{+}$decay modes from the standard $d=5$ operators (Fig. 3), with the same or similar SUSY spectrum as above (see discussion in the next sub-section).

There are three special features of the new $d=5$ operators that are worth noting: 
(1) Unlike the standard $d=5$ operators (Fig. 3), the new $d=5$ operators (Fig. 4) are free from the doublet-triplet splitting problem, even if the effective symmetry is SUSY SO(10) rather than a string-unified $G(2,2,4)$-symmetry.

(2) By the same token, the new $d=5$ operators are independent of $M_{\text {eff }}$ (see Eq. (29)).

(3) Together with the $\bar{\nu} K^{+}$and $\bar{\nu} \pi^{+}$modes, as a distinguishing feature, the new $d=5$ operators lead to the $\mu^{+} K^{\circ}$-decay mode that is typically quite prominent, more so than what one would expect from the standard $d=5$ operators, even in the case of SUSY SO(10) (see Ref. [25]). Specifically, one would expect:

$$
\left[\Gamma\left(p \rightarrow \mu^{+} K^{\circ}\right) / \Gamma\left(p \rightarrow \bar{\nu} K^{+}\right)\right]_{\text {new } d=5} \approx(5-50) \%
$$

This is to be compared with an expected branching ratio of about (5-10)\% for the case of standard $d=5$ operators for SUSY SO(10), and about $10^{-3}$ for SUSY SU(5) (see Eq. (27)). Thus, the $\left(\mu^{+} K^{\circ}\right)$-mode can serve as a signature for the new $d=5$ operators. Observation of a large branching ratio of the $\left(\mu^{+} K^{\circ}\right)$-mode (compared to the $\left(\bar{\nu} K^{+}\right)$-mode) of $(30-50) \%$ (say) would be a clear signal for the relevance of the neutrino-mass related new $d=5$ operators for proton decay, in the context of a SUSY $S O(10)$ or string- $G(2,2,4)$ model in $4 \mathrm{D}$. That would be a valuable piece of information.

Before considering a sharpening of the proton decay lifetimes based on recent works [26], a general comment about the gauge-boson-mediated $d=6$ operator that yields the $\left(e^{+} \pi^{\circ}\right)$ mode as the dominant one, is worth making. While, as mentioned before, naively we expect $M_{X}$ to lie below the unification scale $M_{U} \simeq 2 \times 10^{16} \mathrm{GeV}$, in case $M_{X}$ is as high as about $(1.5-1.7) \times 10^{16} \mathrm{GeV}$, not quite in accord with the naive expectations, $\Gamma^{-1}\left(p \rightarrow e^{+} \pi^{\circ}\right)$ may well be as high as $\approx(5-10) \times 10^{35} \mathrm{yrs}$ (see Eq. (23)). In this case, the $d=5\left(p \rightarrow \bar{\nu} K^{+}\right)$ decay mode (depending on the SUSY spectrum) may well be the dominant mode with lifetime $\approx($ few to 10$) \times 10^{34} \mathrm{yrs}$.

It should be stressed, however, that the $e^{+} \pi^{0}$-mode is the common denominator of all GUT models $(S U(5), S O(10)$, etc.) which unify quarks and leptons and the three gauge forces. Its rate is determined essentially by the matrix element $\alpha_{H}$ and the mass of the $(X, Y)$ gauge bosons related to the SUSY unification scale, without the uncertainty of the SUSY spectrum. I should also mention that the $e^{+} \pi^{0}$-mode is predicted to be the dominant mode in the flipped $S U(5) \times U(1)$-model [129], and also as it turns out in certain higherdimensional orbifold GUT-models, discussed in Sec. 3.1 (see Refs. [99]100]), as well as in a model of compactification of M-theory on a manifold of $G_{2}$ holonomy [153]. For these reasons, intensifying the search for the $e^{+} \pi^{0}$-mode to the level of sensitivity of about (a few) $\times 10^{35}$ years in a next-generation proton decay detector and, if need be, to that of $10^{36} \mathrm{yrs}$ in a next-to-next generation detector, should be well worth the effort.

I will now discuss recent works, which yield expected upper limits on the lifetimes for the $\left(e^{+} \pi^{\circ}\right)$ and $\left(\bar{\nu} K^{+}\right)$decay modes within a class of well-motivated supersymmetric $\mathrm{SO}(10)$ models. 


\subsection{Constraining Proton Lifetime in SUSY SO(10) with Stabilized Doublet- Triplet Splitting}

Following the preliminary discussion on the three mechanisms for proton decay noted in the preceding sub-section, I would now present the gist of a more recent work [26] and its update 27] on sharpening the inverse rates of proton decay induced by the $d=6$ and $d=5$ operators exhibited in Figs. 2 and 3 respectively. The former leads to $e^{+} \pi^{\circ}$ and comparable $\bar{\nu} \pi^{+}$, as the dominant decay modes, while the latter leads to $\bar{\nu} K^{+}$as the dominant mode. The works of the two references cited above, incorporating the LHC searches for SUSY, allow one to set conservative upper limits for the inverse rates of both the $p \rightarrow e^{+} \pi^{\circ}$ and $p \rightarrow \bar{\nu} K^{+}$decay modes within a well-motivated class of SUSY SO(10)-models, based on a minimal set of low-dimensional Higgs multiplets of the type presented in Eq. (9). This comes about through the following set of steps:

(1) First, recognizing that the doublet-triplet (D-T) splitting, requiring a large hierarchy of some 13 orders of magnitude between the masses of the doublet and the triplet, mentioned in the preceding sub-section, poses a major issue for all SUSY GUT models (SU(5), SO(10), $\mathrm{E}_{6}$ etc.), Babu, Tavartkiladze and I attempted to ensure: (a) that the D-T splitting which is naturally induced by the missing VEV mechanism of Refs. [104,105], with $45_{\mathrm{H}}$ having a $\mathrm{VEV}=i \sigma_{2} \otimes \operatorname{Diag}(a, a, a, 0,0)$ along the (B-L)-direction, is stable to a very high accuracy in the presence of all allowed higher dimensional operators; (b) that there does not exist any undersirable pseudo-Goldstone bosons; and (c) that there are no flat directions which would lead to VEVs of fields undetermined. Furthermore, (d) one must also examine by including all GUT-scale threshold corrections to the gauge couplings, the implications of D-T splitting on coupling unification and on proton decay. While some of these issues had been partially addressed in the literature, and a major progress was made in Ref. [154, simultaneous resolution of all four issues had remained a challenge before the work of Ref. [26].

(2) A predictive class of SUSY SO(10) models based on a minimal low-dimensional Higgs system (that includes the multiplets of Eq. (9), together with an additional pair of $16^{\prime}+16^{\prime}$ bar[154] and two SO(10)-singlets) was introduced in Ref. [26], in which all the issues of D-T splitting mentioned above are resolved, and the threshold corrections to the gauge couplings and their implications for proton decay are properly studied as well. A postulated anomalous $\mathrm{U}(1)_{\text {A }}$ gauge symmetry, together with a discrete symmetry $Z_{2}$, both of which may have a string-origin, plays a crucial role in achieving the desired results mentioned above.

(3) The minimal low-dimensional Higgs system lead to smaller threshold corrections unlike in the case of higher dimensional multiplets (like $126_{\mathrm{H}}, \overline{126}_{\mathrm{H}}, 210_{\mathrm{H}}$, possible $54_{\mathrm{H}}$ ). It turns out that within such a low-dimensional Higgs system, subject to the symmetry as mentioned above, there are a large set of cancellations between different GUT-scale threshold contributions (see Ref. [26] for explanation). As a result, somewhat surprisingly, the GUTscale threshold corrections to $\alpha_{3}\left(m_{z}\right)$ are determined in terms of a very few parameters. This makes the model rather predictive for proton decay.

As a novel feature, by incorporating D-T splitting as indicated above and GUT-scale threshold corrections, we find an interesting inverse correlation between the mass-scales $M_{X}$ and $M_{\text {eff }}$, which respectively control the $d=6\left(p \rightarrow e^{+} \pi^{\circ}\right)$ and $d=5\left(p \rightarrow \bar{\nu} K^{+}\right)$decay 
amplitudes (see Eqs. (22) and (24)), of the following form [26]:

$$
M_{\text {eff }} \propto K_{\text {SUSY }}\left[10^{16} \mathrm{GeV} / M_{X}\right]^{3}
$$

Here $K_{\text {SUSY }}$ depends (rather mildly) on the SUSY spectrum at the EW scale, and also on a ratio of two GUT-scale masses. The ratio is varied within a wide range (within reason [26]) so as to get conservative upper limits on the lifefitmes (see below).

Now the empirical lower limit on $\Gamma^{-1}\left(p \rightarrow \bar{\nu} K^{+}\right)$sets a lower limit on $M_{\text {eff }}$, corresponding to any given SUSY spectrum. That in turn yields, via the inverse correlation given in Eq. (32), a theoretical upper limit on $M_{X}$ and thereby on $\Gamma^{-1}\left(p \rightarrow e^{+} \pi^{0}\right)$. Likewise, the empirical lower limit on $\Gamma^{-1}\left(p \rightarrow e^{+} \pi^{0}\right)$ yields, via the correlation Eq. (32), a theoretical upper limit on $\Gamma^{-1}\left(p \rightarrow \bar{\nu} K^{+}\right)$. This chain of arguments thus allows the unusual result leading to predicted upper limits (corresponding to any given SUSY spectrum) on the inverse rates for proton decaying via both the $e^{+} \pi^{0}$ and the $\bar{\nu} K^{+}$modes. Interestingly, as discussed below, these upper limits turn out to be at striking distance from the current empirical lower limits suggesting that proton decay ought to be discovered in the next-generation experiments.

To be quantitative on the predictions mentioned above, we first need the empirical lower limits on proton decay lifetimes. Based on the currently most sensitive searches at SuperKamiokande, the limits on the inverse rates of the two dacay modes are given by [76, 77]:

$$
\begin{aligned}
\Gamma^{-1}\left(p \rightarrow e^{+} \pi^{0}\right)_{\text {expt }} & >1.6 \times 10^{34} \mathrm{yrs} \\
\Gamma^{-1}\left(p \rightarrow \bar{\nu}+K^{+}\right)_{\text {expt }} & >6.6 \times 10^{33} \mathrm{yrs}
\end{aligned}
$$

On the theoretical side, to derive a lower limit on $M_{\text {eff }}$ by using the empirical lower limit on $\Gamma^{-1}\left(p \rightarrow \bar{\nu} K^{+}\right)$, we need two things: (i) the relevant Yukawa couplings, and (ii) the SUSY spectrum, both of which enter into Fig. 3. Now the Yukawa couplings (including their phases) get determined by relating the predictive $\mathrm{SO}(10)$-framework to the masses and mixings of all fermions including neutrinos, and to the observed $\mathrm{CP}$ violation[26].

The major unknown at present is the SUSY spectrum. At the same time, as noted in Sec. 3, the motivations for low-energy supersymmetry in some form seem to be compelling. These include in particular: (i) the need to understand the smallness of the Higgs mass compared to the GUT or Planck scale (or equivalently that of the gauge-hierarchy ratio $\left.\left(m_{W} / M_{U}\right) \sim 10^{-14}\right)$, without introducing unnatural extreme fine-tuning; and (ii) preserving gauge coupling unification with a successful prediction of the weak angle, which calls for both supersymmetry and grand unification, as discussed in Sec. 3.

That said, consistent with the LHC-1 and LHC-2 searches, the Higgs boson mass, and flavor and CP-violating processes, there are, however, different possibilities for the SUSY spectrum that would be compatible with reasonable "SUSY naturalness". The latter corresponds to avoiding unnatural fine-tuning in the Higgs mass. For a good discussion of these possibilities, see e.g. Ref. [155], and references there in..

For our purposes, we adopt two guidelines: (a) simple-minded reasonable SUSY naturalness that suggests a light stop with a mass $\sim(500-1000) \mathrm{GeV}$, say, together with a lighter higgsino that may need to be close to the stop-mass for consistency with LHC searches (see 
e.g. Ref. [151]), and (b) a simple solution to the supersymmetric FCNC and CP problems that suggests heavy sfermions $(\sim(15-20)$ Tev, say) of the first two generations. Such an inverted hierarchy spectrum for the sfermions together with a light stop has been considered by many authors, see e.g. Refs. [150] and [156]. As mentioned before, for concreteness, we essentially follow the work of Badziak, Dudas, Olechowski and Pokorski[150], which is cast within the GUT-framework. An inverted hierarchy of the type mentioned above can be obtained at the electroweak scale by using partial universality in the soft parameters at the GUT-scale as follows:

$$
\begin{aligned}
m_{\mathrm{o}}(1,2) \sim(15-25) \mathrm{TeV} & \gg m_{\mathrm{o}}(3) \sim(3-3.8) \mathrm{TeV} \gg m_{1 / 2} \sim(1.2-2) \mathrm{TeV}, \\
\text { with } m_{\mathrm{o}}(H u) & =m_{\mathrm{o}}(3) \neq m_{\mathrm{o}}(H d) ; A_{\mathrm{o}}=0 \text { to }-2 \mathrm{TeV}
\end{aligned}
$$

The $\mu$-parammeter (at EW scale) is determined by radiative electroweak symmetry-breaking condition to be $\sim(500-800) \mathrm{GeV}$, with $\tan \beta=10$, as input. The inverted hierarchy in the soft masses as shown above can be realized consistently through the use of flavor symmetries (like the $\mathrm{Q}_{4}$-symmetry in our case [26], and an Abelian $U(1)$-symmetry in [150]), which distinguish between the third family and the first two). Input parameters as in Eq. (35) lead to a mass-pattern at the EW scale as follows (only a few relevant masses are listed):

$$
\begin{aligned}
m_{h} & \approx 125 \mathrm{GeV}, \quad m_{\tilde{t}_{1}} \sim(500-1000) \mathrm{GeV}, \\
m_{\tilde{t}_{2}} & \sim(1.5-2.2) \mathrm{TeV}, \quad m_{\tilde{q}_{1,2}} \sim(18-21) \mathrm{TeV}, \\
m_{\tilde{b}_{1,2}} & \sim(1.8-3) \mathrm{TeV}, \quad m_{\tilde{l}_{1,2}} \sim(18-21) \mathrm{TeV}, \\
m_{\tilde{l}_{3}} & \sim(3.1-3.5) \mathrm{TeV}, \quad m_{\tilde{\chi}_{i}} \approx m_{\tilde{B}} \sim(460-800) \mathrm{GeV}, \\
m_{\tilde{W}} & \sim(830-1200) \mathrm{GeV}, \quad m_{\tilde{g}} \sim(2.5-3.5) \mathrm{TeV},
\end{aligned}
$$

Note the possibility of a light stop $(\sim(500-1000) \mathrm{GeV})$ with a higgsino/bino being lighter but close to it within about $(20-100) \mathrm{GeV}$, which seems to be consistent with the LHC13 searches 148 149, 151]. Spectra of the type presented above have been shown[150] to be consistent with $\mathrm{BR}(\mathrm{b} \rightarrow \mathrm{s} \gamma)$, with either the lightest higgsino or an almost pure bino being the LSP dark matter, and $\operatorname{BR}\left(B_{S} \rightarrow \mu^{+} \mu^{-}\right)$.

The fine-tuning parameter may be defined as in Ref. [150] by $\Delta \equiv \max \left\{\Delta_{a}\right\}$, where $\Delta_{a} \equiv\left|\frac{\partial\left(\ln m_{h}\right)}{\partial(\ln a)}\right|$; here "a" represents any soft term or $\mu$. Spectra of the type given above correspond to $\Delta \approx 150-250$. We regard this as reasonable naturalness (in contrast to extreme fine-tuning).

Using the SUSY spectrum of the type given in Eq. (36), and the empirical lower limits on proton decay lifetimes given in Eqs. (33) and (34), one can now utilize the correlation Eq. (32) to calculate the upper limits on the inverse rates for the proton decaying via both the $e^{+} \pi^{0}$ and the $\bar{\nu} K^{+}$modes. To arrive at conservative values for these upper limits, we allow for a wide variation in the ratio of the GUT-scale masses that enters into $K_{\mathrm{SUSY}}$ (of Eq. (32))[26]. We also consider some variation in the SUSY spectrum relative to the type exhibited in Eq. (36), including the possibility that the sfermions of the first two families have medium-heavy $(\sim 4 \mathrm{TeV})$ rather than very heavy $(\sim 20 \mathrm{TeV})$ masses, always requiring reasonlable SUSY naturalness and $m_{h} \approx 125 \mathrm{GeV}$. In addition we allow uncertainties in 
the lattice-value of the matrix elements and $\alpha_{3}\left(m_{z}\right)$. Including these uncertainties, the correlation equation and the empirical lower limits on the proton decay lifetimes yield the following theoretical upper limits for the same:

$$
\begin{aligned}
& \Gamma^{-1}\left(p \rightarrow e^{+} \pi^{0}\right)_{\text {Theory }} \lesssim(2-10) \times 10^{34} \text { Yrs } \\
& \Gamma^{-1}\left(p \rightarrow \bar{\nu} K^{+}\right)_{\text {Theory }} \lesssim(1-8) \times 10^{34} \text { Yrs }
\end{aligned}
$$

These should be regarded as conservative upper limits because the uncertainties of the type mentioned above are all stretched together so as to prolong the proton lifetimes. The actual lifetimes can be quite a bit shorter than the upper limits exhibited above.

As we see, the predicted upper limits (Eq. (37)) are within factors of five to ten above the current SuperKamiokande limits (Eqs. (33), (34)). I should add that supersymmetric grand unified theories that are in accord with the observed masses and mixings of all fermions, including neutrinos, typically yield estimated proton lifetimes in the range as mentioned above (see Ref. [75] for an overview). Thus, the prospects for discovery of proton decay in the nextgeneration deep underground detectors - including especially the $560 \mathrm{kt}$ water Cherenkov detector at HyperKamiokande and the (20-70) kt Liquid Argon detector at LBNF-DUNE would be high.

\subsection{Proton Decay as a Unique Probe to Physics at Ultrashort Distances}

Proton decay, if discovered, would provide a unique window to view physics at truly high energies $\sim 10^{16} \mathrm{GeV}$, or equivalently at truly short distances $\sim 10^{-30} \mathrm{~cm}$. This cannot be realized through accelerators/colliders in the conceivable future. To be specific, some of the valuable insights which one may gain through the discovery and subsequent study of proton decay include the following:

(i) If $p \rightarrow \bar{\nu} K^{+}$decay mode is seen with an inverse decay rate $\sim\left(10^{34}\right.$ to (a few $) \times 10^{35}$ ) yrs, say, in the context of higher unification in $4 \mathrm{D}$, it would imply that either the standard $d=5$ (Fig. 3) or the new $d=5$ operator (Fig. 4), or both, are relevant, involving physics at the unification-scale $\sim 2 \times{ }^{16} \mathrm{GeV}$. Importantly, it would mean that supersymmetry in some form should exist at low energies. (The latter, hopefully, may be discovered at the LHC in the meantime).

(ii) If $p \rightarrow \mu^{+} K^{\circ}$ decay is seen with a decent branching ratio $(\gtrsim 30 \%)$, in the context of a gauge-unification of forces in $4 \mathrm{D}$, it would mean (as discussed in Sec. 7.1) that neither the $d=6$ (Fig. 2) nor the standard $d=5$ operator (Fig. 3) can account for such an observation. And, the neutrino-mass related new $d=5$ operators [24,25] must be playing a role in proton decay; that would mean proton decay knows about the origin of neutrino masses and vice versa! As noted in the later part of Sec. 3, the $p \rightarrow \mu^{+} K^{\circ}$ decay mode can be prominent or dominant through $\mathrm{d}=6$ operators within a certain higher dimensional orbifold GUT models as well ( see the last four papers in Ref. [99]).

(iii) If (B-L)-violating decay modes of the nucleon[157] such as $n \rightarrow e^{-} \pi^{+}$or $p \rightarrow e^{-} \pi^{+} \pi^{+}$ (satisfying $\Delta(\mathrm{B}-\mathrm{L})=-2$ ) are seen at all (a feature which I have not discussed), it 
would mean that fundamental physics at intermediate scales $\ll M_{U} \approx 2 \times 10^{16} \mathrm{GeV}$ is necessarily present. This would, of course, be incompatible with the striking successes of the "conventional" picture of one-step breaking of SUSY-GUT like SUSY-SO(10) to the SM at $M_{U}$, including those of the prediction of the weak angle (Sec. 3) and an understanding of the mass-scales of neutrino-oscillations (Sec. 6). In this sense, observation of (B-L)-violating decay modes of the proton would imply that the successes as above are, somehow, accidents. Yet, experiment is the final arbiter. Thus, one must keep an open mind and search for such decay modes as sensitively as possible. Nonobservation of such decay modes would, of course, serve to strengthen the conventional picture.

(iv) If $p \rightarrow e^{+} \pi^{\circ}$ decay is seen with a decent inverse rate $\left(\lesssim 10^{36}\right.$ yrs, say), then in the context of gauge-unification in $4 \mathrm{D}$, it would imply that the gauge-mediated $d=6$ operator (Fig. 2) is very likely relevant for the decay. That would mean that not only $q \leftrightarrow l$ but also $q \leftrightarrow \bar{q}$ and $q \leftrightarrow \bar{l}$ unifications are relevant. In the context of unification through symmetries like $\mathrm{SO}(10), \mathrm{SU}(5)$ or a string-unified $G(2,2,4)$-symmetry in $4 \mathrm{D}$, it would in turn imply that an intact GUT-symmetry like $\mathrm{SO}(10)$ or $\mathrm{SU}(5)$, rather than a string-unified $G(2,2,4)$-symmetry (which is on par with $\mathrm{SO}(10)$ in explaining observed neutrino oscillations[25] $]^{26}$ is very likely operative in 4D. SUSY SU(5) would yield $p \rightarrow e^{+} \pi^{\circ}$-decay with an inverse rate as above; it however seems to be disfavored on other grounds, especially by observed neutrino oscillations (see Sec. 6). Thus, at least in the context mentioned above, observation of the $p \rightarrow e^{+} \pi^{\circ}$ decay mode would clearly suggest that an intact SUSY SO(10) is operative in 4D. As mentioned at the end of Sec. 7.1, such a decay mode could, of course, also arise in other context such as, flipped $S U(5) \times U(1)$ [129], or certain higher-dimensional orbifold GUT-models as in Ref. [99,100], or string-theory models as in Ref. [153].

(v) On the other hand, if, $p \rightarrow e^{+} \pi^{\circ}$ decay mode is not seen with an inverse decay rate as high as, (say) $\left(10^{36}\right.$-even $\left.10^{37}\right)$ yrs, but $p \rightarrow \bar{\nu} K^{+}$decay mode is seen with an inverse rate $\lesssim 10^{35}$ yrs, it would first of all mean, at least in the context of a gauge-unification of forces in $4 \mathrm{D}$, that an intact GUT-symmetry like $\mathrm{SO}(10)$ is very likely not operative in $4 \mathrm{D}$; instead an effective symmetry like $G(2,2,4)$, or its close relative $G(2,1,4)$, very likely having a string-origin, is operative in $4 \mathrm{D}$ with low-energy supersymmetry; and it is the neutrino-mass related $d=5$ operator (see Sec. 7.1) that induces such a decay.

In short, proton decay, if seen, will bring a wealth of knowledge of a fundamental nature that can not be gained by any other means.

As we have seen, the potential for discovery of proton decay, within a well-motivated class of grand unification models, is high. This is why an improved search for proton decay is now most pressing. This can only be done with a large detector built deep underground. Most desirably we would need both Water Cherenkov (as in HyperKamiokande) and Liquid

\footnotetext{
${ }^{26}$ Within an effective $G(2,2,4)$-symmetry, the exchange of a scalar $(1,1,6)$-multiplet having a GUT-scale mass can induce the $p \rightarrow e^{+} \pi^{\circ}$ decay, but such a decay mode will not be a compelling feature of the symmetry.
} 
Argon (as in LBNF-DUNE) detectors, because the former is specially sensitive to the $\left(e^{+} \pi^{\circ}\right)$ mode, and the latter to the $\left(\bar{\nu} K^{+}\right)$-mode. Such a detector, coupled to a long-baseline intense neutrino beam, can simultaneously sensitively study neutrino oscillations so as to shed light on neutrino mixing parameters, mass-ordering, and most importantly $\mathrm{CP}$ violation in the neutrino system. And it can help efficiently study supernova neutrinos. In short such a detector (or rather two such detectors with the HyperKamiokande being specially sensitive to the $e^{+} \pi^{0}$ mode of proton decay and the DUNE/LBNF to the $\bar{\nu} K^{+}$mode) would have a unique multi-purpose value with high discovery potential in all three areas.

\section{Concluding Remarks}

Neutrinos seem to be as elusive as revealing. Simply by virtue of their tiny masses, they provide some crucial information on the nature of the unification symmetry and on the unification scale, more precisely on the (B-L)-breaking scale. In particular, as argued in

Sec. 6 , combined with the $b / \tau$ mass-ratio, the mass-scale $\left(\sqrt{\Delta m_{\text {Atm }}^{2}} \approx \sqrt{\Delta m_{31}^{2}} \approx 1 / 20 \mathrm{eV}\right)$ of the atmospheric neutrino oscillation provides a clear support for the following three features:

(i) the existence of the SU(4)-color symmetry in 4D at and above the GUT-scale which provides not only the RH neutrinos but also B-L as a local symmetry and a value for $m\left(\nu_{\text {Dirac }}^{\tau}\right)($ Eq. (6));

(ii) the (B-L)-breaking scale being close to the familiar SUSY unification-scale $M_{U} \approx$ $2 \times 10^{16} \mathrm{GeV}$, which provides the mass-scale $M_{R}$ for the Majorana mass of the heaviest RH neutrino (see Eqs. (12) and (17)); and importantly

(iii) the seesaw mechanism that successfully explains the atmospheric neutrino oscillation mass-scale by utilizing both (i) and (ii) (see Eqs. (18)-(21)).

In turn this chain of argument selects out the effective symmetry in $4 D$ being either a stringderived $G(2,2,4)$ or $S O(10)$-symmetry, as opposed to other alternatives like $S U(5),[S U(3)]^{3}$ or even flipped $S U(5) \times U(1)$. As a corollary, this supports the idea that Nature intrinsically is left-right symmetric (parity-conserving) [59].

Furthermore, the success of the $G(2,2,4) / S O(10)$-based seesaw mechanism in accounting for the neutrino oscillation mass-scales (both atmospheric and solar, see Sec. 6) implies that the masses of both the heavy RH and the light LH neutrinos are Majorana, not Dirac, which violate lepton number by two units. That in turn provides, by utilizing the out-ofequilibrium decays of the RH neutrinos and the electroweak sphaleron process, the promising mechanism of baryogenesis via leptogenesis, which can naturally yield $Y_{B} \approx 10^{-10}$ (see Sec. 6). In short, the neutrinos may well be at the root of our own origin. As a by-product, the needed Majorana nature of the neutrino masses clearly call for most sensitive searches for neutrinoless double beta decay. 
Including the insight gained from the neutrinos as above, we are now in possession of a set of facts which may be viewed as the matching pieces of a puzzle in that all of them can be understood simply by just one idea - that of supersymmetric grand unification. These include: (i) the quantum numbers of all the members in a family including the RH neutrino; (ii) the quantization of electric charge, with $Q_{e-}=-Q_{p}$; (iii) the dramatic meeting of the three gauge couplings (Fig. 1, right panel) or equivalently the success of the associated prediction of the weak angle; (iv) $m_{b}^{\circ} \approx m_{\tau}^{\circ} ;(\mathrm{v}) \sqrt{\Delta m_{\text {atm }}^{2}} \approx 1 / 20 \mathrm{eV} ;\left(\right.$ vi) a nearly maximal $\theta_{23}^{\nu} \approx \pi / 4$ with a minimal $V_{\mathrm{cb}} \approx 0.04$; and (vii) baryogenesis via leptogenesis leading to $Y_{B} \approx 10^{-10}$ (see Sec. 6).

All of these features and more hang together within a single unified framework based on a presumed string-derived $G(2,2,4)$ or $\mathrm{SO}(10)$ symmetry, with low-energy supersymmetry. Unless this neat fitting of all the pieces within a single simple picture is just a mere coincidence, it is hard to think that that can be the case, it is pressing that dedicated searches be made to find the two missing pieces of this picture - that is: proton decay and supersymmetry. I should add (as mentioned before) that low-energy supersymmetry is motivated independently of gauge coupling unification in that it provides a natural resolution of the gauge-hierarchy problem as well as a viable candidate for cold dark matter. The search for supersymmetry, which is now in progress at the LHC, thus needs to be continued as intensely as possible at the LHC and beyond to cover the multi-TeV region, if need be, at future accelerators and linear colliders. That for proton decay, as noted in the previous section, needs megaton-size deep-underground detectors, like HyperKamiokande and LBNF-DUNE and their successors (if need be), not only to search for this process as sensitively as possible, but also to study the branching ratios of different decay modes, should proton decay be discovered. As discussed in the previous section, the prospects for discovery of proton decay with improvements of current SuperKamiokande limits by factors of 5 to 10 are high.

The discovery of proton decay will no doubt constitute a landmark in the history of physics. That of supersymmetry will do the same. The discovery of these two features supersymmetry and proton decay — will fill the two missing pieces of a pretty picture - a gauge unification of matter and of its forces.

On the theoretical side, it is but natural to dream that a deeper fundamental theory should provide a unity of all the forces of nature including gravity, together with a good quantum theory of gravity, while providing a predictive and realistic description of the physical world. The string/M-theory, with its majestic beauty (in the words of Edward Witten), is undoubtedly the best candidate we now have for such a deeper fundamental theory.

Notwithstanding the limited understanding we presently have of this theory (especially in its non-perturbative aspects), because of the hanging-together of several pieces within one and the same picture as mentioned above, it stands to reason to ask: can a "preferred solution" of string/M-theory (if it could exist) lead to a grand-unified picture as above based on an effective $G(2,2,4)$ or $\mathrm{SO}(10)$-like symmetry in $4 \mathrm{D}$, with the necessary ingredients to go with reality? If such a solution does emerge, it would no doubt provide a very useful and desirable bridge between string theory and the low-energy world described by the standard model. This is because, as noted above, such a bridge seems to work in explaining a set of phenomena in a non-trivial manner. Despite its successes, however, the answers to some 
fundamental issues — such as the origin of the three families with their hierarchical masses and mixings and an understanding of the observed value of the dark energy ( cosmological constant) - are outside of its reach. May be a string-derived grand-unified theory as above could provide a resolution of at least some of these major issues and provide a rationale for the choice of such an effective theory in 4D. Could some clear glimpses of such an utopian picture, based on developments in experiments and theory, with both proton decay and supersymmetry in hand, and with a better understanding of string theory describing reality in our possession, emerge by the 120th birthday of Abdus Salam or even by the 150th? A fulfillment of some or all of these wishes, no matter how compelling they may appear to be, depends of course on what Nature has in store for us?

\section{Acknowledgement}

I would like to thank especially Kaladi Babu, Alon Faraggi, Rabindra Mohapatra, Zurab Tavartkiladze and Frank Wilczek for collaborative discussions over the years which have shaped the content of this article. I have greatly benefitted from discussions with Marcin Badziak, Pasquale Di Bari, Michael Peskin, Pran Nath, Stuart Raby, Goran Senjanovic, Qaisar Shafi and Edward Witten on various aspects of the physics in the paper. Communications from Edward Kearns and M. Shiozawa on the latest SuperKamiokande results on searches for proton decay have been helpful.

I am deeply grateful to late Abdus Salam for the joy of a fruitful collaboration and for a warm friendship that lasted between us till he parted from this world. With gratitude and respect I dedicate this talk to his memory.

I am thankful to Lars Brink, Mike Duff and Kok Khoo Phua for their hospitality, to Chee-Hok Lim and the members of the Stallion Press, Chennai, India for their help in the processing of this paper, and especially to Nikita Blinov, Michael Peskin and Brian Shuve as regards the latter.

\section{References}

[1] The present paper is an expanded version of the talk given by the author which has recently appeared as a contribution to: "The Memorial Volume on Abdus Salam's 90th Birthday”, pp. 31-93 (2017), Ed. by Lars Brink, Michael Duff, and Kok Khoo Phua, Publ. by World Sc.

[2] Source: "Ideals and Realities" — Selected Essays by Abdus Salam, Ed. by C.H. Lai, Publ. by World Scientific.

[3] J. C. Pati in Salam Festschrift, " Twenty Years Later: Why I still Believe in SU(4)color" Ed. by A. Ali, J. Ellis and S. Randjbar-Daemi, Publ. by World Scientific, pp. 368-391, 1993. 
[4] J. C. Pati, "Recollections of Abdus Salam: Scientist and Humanitarian" — Physics Today (abridged version), August (1997); News Letter of the American Chapter of the Indian Physics Association (1997).

[5] J. C. Pati, "With Neutrino Masses Revealed, Proton Decay is the Missing Link", hepph/9811442; Proc. Salam Memorial Meeting (1998), World Scientific; Int'l Journal of Modern Physics A, vol. 14, 2949 (1999).

[6] J. C. Pati and Abdus Salam, a draft on "Ideas on Higher Unification Based on SU(4)color". An extract from the draft was presented in the Rapporteur talk by J.D. Bjorken, Proc. 15th High Energy Conference, Batavia, Vol. 2, p. 301 (1972).

[7] J. C. Pati and Abdus Salam," Unified Lepton - Hadron Symmetry and a Gauge Theory of the Basic Interactions", Phys. Rev. D 8, 1240 (1973).

[8] J. C. Pati and Abdus Salam," Is Baryon Number Conserved?", Phys. Rev. Lett. 31, 661 (1973).

[9] J. C. Pati and Abdus Salam, " Lepton Nymber as the Fourth Color" Phys. Rev. D 10, 275 (1974).

[10] H. Georgi and S. L. Glashow, " Unity of All Elementary Particle Forces ", Phys. Rev. Lett. 32, 438 (1974).

[11] H. Georgi, in Particles and Fields, ed. by C. Carlson (AIP, NY, 1975), p. 575; H. Fritzsch and P. Minkowski, Ann. Phys. 93, 193 (1975).

[12] The SuperKamiokande Collaboration: "Evidence For Oscillation of Atmospheric Neutrinos", ICRR-Report-422-98-18 (July, 1998); Y. Fukuda et al. (Super-Kamiokande), Phys. Rev. Lett. 81, 1562 (1998), hep-ex/9807003; K. Nishikawa (K2K) Talk at Neutrino 2002, Munich, Germany.

[13] Q. R. Ahmad et al. (SNO), Phys. Rev. Lett. 81, 011301 (2002); B. T. Cleveland et al. (Homestake), Astrophys. J. 496, 505 (1998); W. Hampel et al. (GALLEX), Phys. Lett. $B$ 447, 127, (1999); J. N. Abdurashitov et al. (SAGE) (2000), astroph/0204245; M. Altmann et al. (GNO), Phys. Lett. B 490, 16 (2000); S. Fukuda et al. (SuperKamiokande), Phys. Lett. B 539, 179 (2002). Disappearance of $\bar{\nu}_{e}$ 's produced in earth-based reactors is established by the KamLAND data: K. Eguchi et al., hep-ex/0212021.

[14] P. Minkowski, Phys. Lett. B 67, 421 (1977); M. Gell-Mann, P. Ramond and R. Slansky, in: Supergravity, eds. F. van Nieuwenhuizen and D. Freedman (Amsterdam, North Holland, 1979) p. 315; T. Yanagida, in: Workshop on the Unified Theory and Baryon Number in the Universe, eds. O. Sawada and A. Sugamoto (KEK, Tsukuba) 95 (1979); S. L. Glashow, in Quarks and Leptons, Cargése 1979, eds. M. Levy et al. (Plenum 1980) p. 707, R. N. Mohapatra and G. Senjanovic, Phys. Rev. Lett. 44, 912 (1980).

[15] M. Fukugita and T. Yanagida, Phys. Lett. B 174, 45 (1986); M. A. Luty, Phys. Rev. D 45, 455 (1992); G. Lazarides and Q. Shafi, Phys. Lett. B 258, 305 (1991); W. Buchmuller and M. Plumacher, Phys. Lett. B 389, 73 (1996). 
[16] V. Kuzmin, Va. Rubakov and M. Shaposhnikov, Phys. Lett. B 155, 36 (1985).

[17] F. Gursey, P. Ramond and R. Slansky, Phys. Lett. B 60, 177 (1976); Y. Achiman and B. Stech, Phys. Lett. B 77, 389 (1978); Q. Shafi, Phys. Lett. B 79, 301 (1978); A. deRujula, H. Georgi and S. L. Glashow, 5th Workshop on Grand Unification, edited by K. Kang et al., World Scientific, 1984, p. 88.

[18] H. Georgi, H. Quinn and S. Weinberg, "Hierarchy of Interactions in Unified Gauge Theories", Phys. Rev. Lett. 33, 451 (1974).

[19] S. Dimopoulos, S. Raby and F. Wilczek, Phys. Rev. D 24, 1681 (1981); S. Dimopoulos and H. Georgi, Nucl. Phys B 193, 150 (1981); L. Ibanez and G. G. Ross, Phys. Lett. B 105, 439 (1981); N. Sakai, Z. Phys. C 11, 153 (1981); W. Marciano and G. Senjanovic, Phys. Rev. D 25, 3092 (1982) and M. Einhorn and D. R. T. Jones, Nucl. Phys. B 196, 475 (1982). For work in recent years, see P. Langacker and M. Luo, Phys. Rev. D 44, 817 (1991); U. Amaldi, W. de Boer and H. Furtenau, Phys. Rev. Lett. B 260, 131 (1991); F. Anselmo, L. Cifarelli, A. Peterman and A. Zichichi, Nuov. Cim. A 104 1817 (1991).

[20] Y. A. Golfand and E. S. Likhtman, JETP Lett. 13, 323 (1971). J. Wess and B. Zumino, Nucl. Phys. B 70, 139 (1974); D. Volkov and V. P. Akulov, JETP Lett. 16, 438 (1972).

[21] Independently of gauge coupling unification, low-energy supersymmetry with superpartners having masses at the $\mathrm{TeV}$-scale is motivated as being perhaps the best idea known to naturally protect the gauge-hierarchy of an input ratio of $\left(m_{\mathrm{w}} / \mathrm{M}_{\mathrm{U}}\right) \sim 10^{-14}$ in the presence of all radiative corrections: E. Witten, Nucl. Phys. B 185, 513 (1981); R. K. Kaul, Phys. Lett. B 109, 19 (1982). M. Dine, W. Fischler and M. Srednicki, Nucl. Phys. B 189, 575 (1981); S. Dimopoulos and S. Raby, ibid. B 192, 353 (1981); R. K. Kaul and P. Majumdar, Nucl. Phys. B 199, 36 (1982).

[22] For a concise overview on GUT, see e.g. S. Raby in Particle Data Group (PDG) Review (2015); For a brief review of GUTs in 4D and a discussion of string-derived and higher dimensional orbifold GUT models, see A. Hebecker and J. Hisano, PDG Review ( 2016); earlier reviews include: P. Langacker, Phys. Rep. 72, 16 (1981); P. Nath and Pavel Fileviez Perez, Phys. Rep. 441, 191 (2007). For a current review of theory and experiments pertaining to baryon number violation in grand-unified theories, see Ref. [75].

[23] M. Green and J. H. Schwarz, Phys. Lett. B 149, 117 (1984); D. J. Gross, J. A. Harvey, E. Martinec and R. Rohm, Phys. Rev. Lett. 54, 502 (1985); P. Candelas, G.T. Horowitz, A. Strominger and E. Witten, Nucl. Phys. B 258, 46 (1985). For introductions and reviews, see: M. B. Green, J. H. Schwarz and E. Witten, "Superstring Theory" Vols. 1 and 2 (Cambridge University Press); J. Polchinski, "String Theory", Vols 1 and 2 (Cambridge University Press).

[24] K. S. Babu, J. C. Pati and F. Wilczek, "Suggested New Modes in Supersymmetric Proton Decay", Phys. Lett. B 423, 337 (1998). 
[25] K. S. Babu, J. C. Pati and F. Wilczek, "Fermion Masses, Neutrino Oscillations and Proton Decay in the Light of the SuperKamiokande" hep-ph/981538V3; Nucl. Phys. B

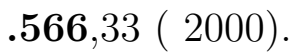

[26] K. S. Babu, J. C. Pati and Z. Tavartkiladze, "Constraining Proton Lifetime in SO(10) with Stabilized Doublet-Triplet Splitting", JHEP 1006, 084 (2010) [arXiv: 1003.2625 [hep-ph]].

[27] K. S. Babu, J. C. Pati and Z. Tavartkiladze, "Update of Ref. [26] in light of LHC", To appear.

[28] S. L. Glashow, Nucl. Phys. 22 57a (1961); S. Weinberg, Phys. Rev. Lett. 19, 1269 (1967); Abdus Salam, in Elementary Particle Theory, Nobel Symposium, ed. by N. Svartholm (Almqvist, Stockholm, 1968), p. 367.

[29] P. W. Higgs, Phys. Lett. 12, 132 (1964); 13, 508 (1964); F. Englert and R. Brout, Phys. Rev. Lett. 13, 321 (1964); G. S. Guralnik, C. R. Hagen and T. W. B. Kibble, Phys. Rev. Lett. 13, 585 (1965); T. W. B. Kibble, Phys. Rev. 155, 1554 (1967).

[30] G. 't Hooft, Nucl. Phys. B 35, 167 (1971); G. 't Hooft and M. Veltman, Nucl. Phys. $B$ 44, 189 (1972).

[31] J. J. Sakurai Ann. Phys. 1, 11 (1960).

[32] C. N. Yang and R. L. Mills, Phys. Rev. 96, 191 (1954).

[33] For attempts to derive the current algebra framework using the singlet U(1) vector gluon see e.g. H. Fritzsch and M. Gell-Mann, Proc. 15th High Energy Conf., Batavia (1972), pages 135-165, and reference there in. The difficulty of SU(9) or SU(12) global symmetry associated with the $\mathrm{U}(1)$ vector gluon coupling was one reason for abandoning this possibility: See J.C. Pati and Abdus Salam, ICTP preprint IC/73/81 (unpublished), and L.B Okun, A.D.Dolgov and V.I Zakharov, Phys. Lett. B 47, 258 (1973).

[34] O. W. Greenberg, Phys. Rev. Lett. 13, 598 (1964).

[35] M. Han and Y. Nambu, Phys. Rev. 139, B1006 (1965).

[36] H. Georgi and S.L. Glashow, Phys. Lett. 28, 1494 (1972).

[37] J. C. Pati and A. Salam (Ref. [6] and [7); H. Fritzsch and M. Gell-Mann, (Ref. [33]); H. Fritzsch, M. Gell-Mann, and H. Leutwyler, Phys. Lett. B 47, 365 (1973).

[38] To clarify the background of this suggestion, as noted in the text, the idea of generating a "superstrong" force by gauging SU(3)-color symmetry was first initiated by Han and Nambu in Ref. [35]. In their work, however, SU(3)-color was broken explicitly, rather than spontaneously, owing to the fundamental coupling of the photon to integercharge quarks (icq); this spoiled the renormalizability of the theory. Furthermore, 
they introduced an additional fundamental strong interaction by utilizing the SU(3)flavor gauge symmetry. Such a possibility is, however, excluded if one uses part of the flavor symmetry to generate electroweak interactions, as in $\mathrm{SU}(2)_{\mathrm{L}} \times \mathrm{U}(1)_{\mathrm{Y}}$. These two shortcomings were removed and the advantages of the combined gauge structure $\mathrm{SU}(2)_{\mathrm{L}} \times \mathrm{U}(1)_{\mathrm{Y}} \times \mathrm{SU}(3)^{\mathrm{c}}$ were noted by Salam and me starting with a contribution to the 1972 Batavia conference [6], followed by a paper in Ref. [7]. See comments on the question of quark-charges in Ref. [39].

[39] At the time of our suggestion of the combined gauge symmetry at the 1972 Batavia Conference (Ref. [6]), Fritzsch and Gell-Mann also noted the possibility of $S U(3)^{c}$ gauging at the same conference [37]. They, and many others, favored the alternative of fractionally charged quarks (fcq) and unbroken SU(3)-color, with permanent confinement of quarks and gluons. With little understanding of confinement in those days, we thought that the case of fcq with permanent confinement should be regarded only as an alternative to the case of spontaneously broken $S U(3)^{c}$, which would lead to gauge integer charge quarks (icq), with possibly semi-confined but ultimately liberated quarks. Despite the presence of fundamental scalars, that are needed to break $S U(3)^{\mathrm{c}}$ spontaneously in the case of icq, the loss of asymptotic freedom and thus departure from scaling are rather mild at the energy scales $(<1-2 \mathrm{GeV})$ probed at that time. The two alternatives arise within the same theory depending upon the nature of SSB. G. Rajasekaran and P. Roy (Pramana 6, 303 (1975)) and Salam and I, (Phys. Rev. Lett. 36, 11 (1976)), as well as others, therefore, proposed key experiments which could distinguish between the two alternatives. Eventually, by the 1980's on the basis of these tests, especially the two-photon experiments (see e.g. R. Godbole, J. Pati, S. Rindani, T. Jayaraman and G. Rajasekaran, Phys. Lett. 142, 91 (1984)), fcq was clearly favored over gauge icq. The basic ideas of higher unification, including the advantages of the symmetry-structure $G(2,2,4)$ listed in the text, as also the success of the (combined) standard model symmetry, are of course independent of the nature of quark-charges.

[40] By the time SU(5) was proposed, about a year later[10], the situation had changed. Asymptotic freedom had been discovered[41, as also the weak neutral current interaction 42], both of which gave strong support to the standard model.

[41] D. Gross and F. Wilczek, Phys. Lett. 30, 1343 (1973); H. D. Politzer, ibid, 30, 1346 (1973).

[42] F. J. Hasert et al., Phys. Lett. B 46, 121, 138 (1973); P. Muset et al., J. Phys. (Paris) 11/12, T34 (1973).

[43] S.L.Adler,Phys.Rev. 177, 2426 (1969); J. S. Bell and R. Jackiw, Nuovo Cimento 60A, 47 (1969).

[44] J. C. Pati and Abdus Salam, Refs. 6] and [7]; C. Bouchiat, J. Iliopoulos and P. Meyer, Phys. Lett. B 38, 519 (1972); S. Weinberg, in Fundamental Interactions in Physics and Astrophysics, eds. G. Iverson et al. (Plenum Press, New York, 1973), p. 157.

[45] S. Glashow, J. Iliopoulos and L. Maiani, Phys. Rev. D 2, 1285 (1970). 
[46] N. Cabibbo, Phys. Rev. Lett. 10, 531 (1963).

[47] M. Kobayashi and K. Masakawa, Prog. Theor. Phys. 49, 282 (1972).

[48] S. Weinberg, Phys. Rev. Lett. 31, 494 (1973); R. N. Mohapatra, J. C. Pati and P. Vinciarelli, Phys. Rev. D 8, 3652 (1973).

[49] J. D. Bjorken, Phys. Rev. Lett. 16, 408 (1966); J. D. Bjorken, Phys. Rev. 179, 1547 (1969).

[50] R. P. Feynman, Phys. Rev. Lett. 23, 1415 (1969); J. D. Bjorken and E. A. Paschos, Phys. Rev. 185, 1975 (1969).

[51] S. Weinberg, Phys. Rev. Lett. 31, 494 (1973); D. J. Gross and F. Wilczek, Phys. Rev. D 8, 3633 (1973); H. Fritzsch, M. Gell-Mann and H. Leutwyler, Phys. Lett. B 47, 365 (1973). The unconventional idea that quarks are somehow confined was first conceived of by M. Gell-Mann from the very early days, prior to the ideas of color and QCD (private communications; this may be inferred from his statement on "mathematical" quarks).

[52] See e.g. M. Creutz, Phys. Rev. Lett. 45, 313 (1980); Phys. Rev. D 21, 2308 (1980); M. Lüscher and P. Weisz, JHEP 0207, 049 (2002); for a review, see e.g. T. DeGrand, Int. J. Mod. Phys. A 19, 1337 (2004).

[53] In general, the G(2,2,4) symmetry can have SU(2) Witten-anomaly (E. Witten, Phys. Lett. $B$ 117,324 (1982)). But, for the usual choice of matter and Higgs multiplets (including the SUSY case), such as $(2,1,4)$ and $(2,2,1)$ etc., the number of $\mathrm{SU}(2)$ doublets of chiral fermions is even; such choices are thus safe. I thank Kaladi Babu for reminding me of the issue.

[54] C. N. Yang, "Necessary Subtlety and Unnecessary Subtlety", Proceedings of the Int'l Conference on "Neutrinos and Implications for Physics Beyond the Standard Model", Ed. by R. Shrock, Publ. By World Scientific, pp5 (October 11, 2002). In this talk, Yang states: "Personally I tend to disbelieve unnecessary subtleties. That is perhaps why Dyson called me conservative. I did not believe in neutrino oscillations, even after Davis' painstaking work and Bahcall's careful analysis: the oscillations were, I believed, uncalled for. Now, after the beautiful experiments which we shall hear about in the next few days, I have to surrender and accept neutrino oscillations, as reality, but still as unnecessary subtleties". Relative to Yang's remark it seems appropriate to mention that neutrino oscillations (and thus neutrino masses) now appear to be a very necessary subtlety of nature in the sense that, (a) as discussed in Sec. 6, the tiny neutrino masses seem to be the nature's way to provide a clue to physics at the unification scale including the nature of the unification symmetry, and (b) they also seem to be at the root of the origin of matter-antimatter asymmetry, and thus at the root of our own origin.

[55] Initially, owing to his fondness for the two-component theory of the neutrino, of which he was a co-father [56], even Salam, despite his liking for SU(4)-color, resisted against 
the introduction of the RH neutrino. Happily, his reservations in this regard softened over a few months. Sadly, though, he did not live to see, the empirical support of these ideas that came through the discovery of the atmospheric neutrino oscillation in 1998.

[56] The two-component theory of the neutrino was first suggested by H. Weyl ( around 1930) to explain the seeming masslessness of the neutrino, but it was rejected by W.Pauli (Handbuch der Physik, Verlag. J. Springer, Berlin, Vol. 24 (1933)) on the ground that that would violate parity. The idea was revived by A. Salam, Nuovo cim. 5, 299 (1957); L. D. Landau, Nucl. Phys. 3, 127 (1957); and T. D. Lee and C. N. Yang, Phys. Rev. 105, 1671 (1957).

[57] S. Weinberg, Phys. Rev. Lett. 43, 1566 (1979); E. Akhmedov, Z. Berezhiani and G. Senjanovic, Phys. Rev. D D 47, 3245 (1993).

[58] J. C. Pati, "Implications of the SuperKamiokande Result on the Nature of New Physics", Proceedings of the Neutrino-98 Conference, held at Takayama, Japan (June 4-9, 1998), Published in Nucl. Phys. Proc. Suppl. 77, 299 (1999).

[59] J. C. Pati and Abdus Salam, Phys. Rev. D 10, 275 (1974); R. N. Mohapatra and J. C. Pati, Phys. Rev. D 11, 566 and 2558 (1975); G. Senjanovic and R. N. Mohapatra, Phys. Rev. D 12, 1502 (1975).

[60] For an incomplete list of references on consideration of TeV-scale $W_{R}$, see e.g., W. Y. Keung and G. Senjanovic, Phys. Rev. Lett. 50, 1427 (1983); A. Ferrari et al., Phys. Rev. D62, 013001( 2000); A. Melfo, hep-ph/0302216; Y. Zhang, H. An, X. Ji and R. N. Mohapatra, Nucl. Phys. B 802, 247 (2008); A. Maiezza, M. Nemevsek, F. Nesti and G. Senjanovic, Phys. Rev. D 82, 055022 (2010); V. Tello, M. Nemevsek, F. Nesti and G. Senjanovic, Phys. Rev. Lett. 106, 151801 (2011); C. H. Lee, P. S. Bhupal DeV and R. N. Mohapatra, Phys. Rev. D 88, no. 9, 093010 (2013); M. Lindner, F. S. Queiroz, W. Rodejohann and C. E. Yaguna, JHEP 1606, 140 (2016); D. Borah, S. Patra and U. Sarkar, Phys. Rev. D 83, 035007 (2011); J. Chakrabortty, H.Z. Devi, S. Goswami and S. Patra, JHEP 1208, 008 (2012); R. L. Awasthi, M. K. Parida and S. Patra, JHEP 1308, 122 (2013); G. Senjanovic and V. Tello, Phys. Rev. Lett. 114, 071801 (2015); J. Brehmer, J. Hewett, J. Kopp, T. Rizzo and J. Tattersal, JHEP 1510, 182 (2015); B. A. Dobrescu and Z. Liu, Phys. Rev. Lett. 115, no. 21, 211802 (2015).

[61] A. Davidson, Phys. Rev. D 20, 776 (1979); R. E. Marshak and R. N. Mohapatra, Phys. Lett. B 91, 222 (1980).

[62] G. 't Hooft, Nucl. Phys.B 79, 276 (1974); A. M. Polyakov, JETP Lett. 20, 194 (1974).

[63] G. Lazarides, M. Magg and Q. Shafi Phys. Lett. B 97, 87 (1980); G. 't Hooft, Nucl. Phys.B 105, 538 (1976). Interestingly, based on topological arguments, the first paper shows that the spontaneous breaking of $S O(10)$ to $G(2,2,4)$ would produce $Z_{2}$ magnetic monopoles with one unit of Dirac charge $\left(g_{D}\right)$, even though $G(2,2,4)$ is nonAbelian, while the next stage involving the breaking of $G(2,2,4)$ to the SM symmetry at an intermediate scale, would produce magnetic monopoles with twice the Dirac charge. 
[64] P. A. M. Dirac, Proc. R. Soc. Lond. A 133, 60 (1931).

[65] A. H. Guth, Phys. Rev. D 23, 347 (1981); A. D. Linde, Phys. Lett. B 108, 389 (1982); A. Albrecht and P. J. Steinhardt. Phys. Rev. Lett. 48, 1220 (1982).

[66] A. Rajantie, Physics Today 69, number 10, 41 (2016); K. A. Olive et al., (Particle Data Group), Chin. Phys. C, 38, 090001 (2014), update (August 2015) by D. Milstead and E. J. Weinberg.

[67] V. N. Senoguz and Q. Shafi, Phys. Lett. B 752, 169 (2016); G. Lazarides and Q. Shafi, Phys. Lett. B 148, 35 (1984).

[68] J. C. Pati, A. Salam and J. A. Strathdee, Nuovo Cim. A 26, 72 (1975); Phys. Lett. $B$ 108, 121 (1982). The maximal local symmetry $\mathrm{SU}(16)$ of sixteen two-component fermions in a family was considered as a possible parent symmetry for grand unification in the early days, despite its being much bigger than its subgroups of SU(5) and SO(10), because it treats $\mathrm{B}$ and $\mathrm{L}$ as local rather than global symmetries and thereby provides a fundamental reason for their origins. These symmetries are thus exact in the lagrangian, but must be violated spontaneously for reasons explained in footnote 12. It turns out that such spontaneous breaking mixes gauge bosons of $\mathrm{SU}(16)$ carrying different $(\mathrm{B}, \mathrm{L})$ quantum numbers and thereby induces proton decays such as $\mathrm{p} \rightarrow \mathrm{e}^{+} \pi^{\circ}$ analogous to the cases of $\mathrm{SU}(5)$ and $\mathrm{SO}(10)$ where the violations are explicit. Symmetries like $\mathrm{SU}(16)$ do need, however, three mirror families (which are chiral and non-trivial under electroweak symmetry) to cancel triangle anomalies. Unlike in the 1970's, these mirror families are constrained, however, by precision electroweak tests [69,70], and, now, excluded by the Higgs-production rate [71], barring the possibility of a richer Higgs-system.

[69] M. Peskin and T. Takeuchi, Phys. Rev. Lett. 65, 964 (1990); Phys. Rev. D 46, 381 (1991).

[70] G. Altarelli, R. Barbieri and S. Jadach, Nucl. Phys. B 369, 3 (1992); G. Altarelli, R. Barbieri and F. Caravaglios, Nucl. Phys. B 405, 3 (1993).

[71] For a review, see M. Vysotsky, arXiv:1312.0474.

[72] W. H. Furry, Phys. Rev. 56, no. 12, p. 1184 (1939).

[73] For a recent review on theory and experiments on neutrinoless double beta-decay, see e.g. S. Dell'Oro et al., Advances in High Energy Physics Vol. 2016 (2016), ID 2162659 , and references there in.

[74] V. Kuzmin, JETP Lett. 12, 228 (1970); S. L. Glashow, Proc. Neutrino 79 Conf., Berg, 2, 518 (1979); R. N. Mohapatra and R. E. Marshak, Phys. Rev. Lett. 44, 1316 (1980). For an overview of theory and phenomenology of $n-\bar{n}$ oscillation, see R.N. Mohapatra, J. Phys. G 36, 104006 (2009).

[75] For a concise review on "Baryon Number Violation" covering theory and experiments on both proton decay and $n-\bar{n}$ oscillation in the context of unified theories see the 
Snowmass Report (2013); arXiv:1311.5285 (hep-ph), Conveners: K.S. Babu and E. Kearns.

[76] For lower limit on $\Gamma^{-1}\left(p \rightarrow e^{+} \pi^{\circ}\right)$ : K. Abe et al. (SuperKamiokande Collaboration), Phys. Rev. D 95, no. 1, 012004 (2017).

[77] For lower limit on $\Gamma^{-1}\left(p \rightarrow \bar{\nu} K^{+}\right)$: K. Abe et al. (SuperKamiokande Collaboration), Phys. Rev. D 90, no. 7, 072005 (2014); Talk presented on behalf of SuperKamiokande collaboration by M. Shiozawa at 34th Intl. Cosmic Ray Conference (ICRC 2016).

[78] T. W. B. Kibble, G. Lazarides and Q.Shafi, Phys. Rev. D26, 435 (1982); D. Chang, R. N. Mohapatra and M. K. Parida. Phys. Rev. Lett., 52, 1072 (1984). For a clear exposition of the role of the generalized C-parity in $S O(10)$ and its correspondence with the L-R discrete symmetry (ordinary parity) [59], that is associated with the $G(2,2,4)$ symmetry, see C. S. Aulakh and A. Girdhar, hep-ph/0204097.

[79] F. Gursey, P. Ramond and P. Sikivie, Phys. Lett. B 60, 177 (1976).

[80] For reviews, see e.g. P. Langacker, talk at Gatlinburg Conference, June 94, hep-ph 9411247, and P. Langacker and N. Polonsky, Phys. Rev. D 52, 3081 (1995), and references there in.

[81] LEP and SLD Average: On the weak angle: G. Gabbiani et al., Phys. Rep. 427, 257 (2006). For LEP measurement on $\alpha_{3}\left(m_{z}\right)$, see W. M. Yao et al. (Particle Data Group, J. Phys. G 33, 1 (2006)).

[82] F. del Aguila and L.E. Ibanez, Nucl. Phys. B 177, 60 (1981); T. G. Rizzo and G. Senjanovic, Phys. Rev. D 25, 235 (1982); T. G. Rizzo and G. Senjanovic, Phys. Rev. Lett. 46, 1315 (1981); D. Chang, R. N. Mohapatra, J. Gipson, R. E. Marshak and M. K. Parida, Phys. Rev. D 31, 1718 (1985); R. N. Mohapatra and M. K. Parida, Phys. Rev. D 47, 264 (1993) hep-ph/9204234]; N. G. Deshpande, E. Keith and P. B. Pal, Phys. Rev. D 46, 2261 (1993); S. Bertolini, L. Di Luzio and M. Malinsky, Phys. Rev. D 85, 095014 (2012) arXiv:1202.0807 [hep-ph]].

[83] D. G. Lee, R. N. Mohapatra, M. K. Parida and M. Rani, Phys. Rev. D 51, 229 (1995) arXiv:hep-ph/9404238.

[84] G. Altarelli and D. Meloni, JHEP 1308, 021 (2013) arXiv:1305.1001 [hep-ph]].

[85] K. S. Babu and S. Khan, Phys. Rev. D 92, 075018 (2015) arXiv:1507.06712 [hep-ph]].

[86] Promising string-theory solutions yielding the $G(2,2,4)$-symmetry in $4 \mathrm{D}$ have been obtained using different approaches, by a number of authors. They include: I. Antoniadis, G. Leontaris, and J Rizos, Phys. Lett. B 245, 161 (1990); G. K. Leontaris, Phys. Lett. B 372, 212 (1996), hep-ph/9601337; A. Murayama and T. Toon, Phys. Lett. B 318, 298 (1993); Z. Kakushadze, Phys. Rev. D 58, 101901 (1998); G. Aldazabal, L. I. Ibanez and F. Quevedo, hep-th/9909172; G. Shiu and S.-H. Henry Tye, Phys. Rev. D58 106007 (1998); C. Kokorelis, hep-th/0203187, hep-th/0209202; M. Cvetic, 
G. Shiu, and A. M. Uranga, Phys. Rev. Lett. 87, 201801 (2001), hep-th/0107143, and Nucl. Phys. B 615, 3 (2001), hep-th/0107166; M. Cvetic and I. Papadimitriou, hepth/0303197; R. Blumenhagen, L. Gorlich and T. Ott, hep-th/0211059. For a type I string-motivated scenario leading to the $G(2,2,4)$ symmetry in $4 \mathrm{D}$, see L. I. Everett, G. L. Kane, S. F. King, S. Rigolin and L. T. Wang, hep-th/0202100. A promising class of four dimensional three-family $G(2,2,4)$-models has been obtained from heterotic orbifold constructions, involving 5D or 6D orbiflold GUT such as $\mathrm{SO}(10)$, by T. Kobayashi, S. Raby, and R. J. Zhang, Nucl. Phys. B 704, 3 (2005), hep-ph/0409098. This class of $G(2,2,4)$ models has subsequently been applied in a set of papers to study fermion masses and mixings, and cosmological issues including inflation and leptogenesis; for a recent application see Z.Poh, S.Raby and Z. Wang, hep-ph/1703.09309, and references there in. Another class of solutions leading to the $G(2,2,4)$-symmetry from Type II-A orientifolds with intersecting D6-branes is obtained in M. Cvetic, T. Li and T. Liu, hep-th/0403061. For attempts to calculate Yukawa couplings within intersecting Dbrane models leading to the G(2,2,4) symmetry, see D. Cremades, L. E. Ibanez, and F. Marchesano, hep-th/ 0302105. For alternative attempts based on flux compactification of Type II-B string theory leading to three-generation $G(2,2,4)$-solutions, with one heavy generation, which satisfy the constraints of K-theory as well as achieve SUSYbreaking and moduli-stabilization, see F. Marchesano and G. Shiu, hep-th/0408059; and hep-th/0409132. More recently, free fermionic heterotic-string vacua leading to $G(2,2,4)$ symmetry in $4 \mathrm{D}$ with three generations and desired symmetry-breaking multiplets have been shown to exist, in which exotic fractionally charged states do not exist in the spectrum below the string scale: see B. Assel, C. Christodoulides, A. E. Faraggi, C. Kounnas and J. Rizos, Phys. Lett. B 683, 306 (2010); "Classification of Heterotic Pati-Salam Models", Nucl. Phys. B 844, 365 (2011). Effective G(2,2,4)-vacua in 4D have also been obtained in the context of Type II B string theory and F-theory by S. Krippendorf, M. Dolan, A. Maharana and F. Quevedo, JHEP 1006.092 (2010).

[87] P. Anastasopoulos, G. K. Leontaris and N. D. Vlachos "Phenomenological Analysis of D-brane Pati-Salam Vacua", arXiv:1002.2937 [hep-th]; For a systematic construction of Gepner-type Orientifold vacua leading to $\mathrm{G}(2,2,4)$ and other models, see P. Anastasopoulos, T. Dijkstra, E. Kirtsis and B. Schellekens, arXiv: hep-th/0605226. See Appendix B.2 of the first paper, in which one of the constructions possesses two $(1,1,15)$ 's, while the one next to it is devoid of $(1,1,6)$, as desired to go with Eq. (8). This suggests that a single solution possessing the desired matter-spectrum and the Higgs multiplets of Eq. (8) together with a light Higgs bi-doublet ( and nothing else) might well be possible, but its construction remains a challenge ( I thank George Leontaris for discussion in this regard).

[88] M. Cvetic, D. Kleverse, D. Kaloni Mayorga Pena, P. K. Oehlman and J. Reuter "Three-Family Particle Physics Models from Global F-theory Compactifications", arXiv:1503.02068 [hep-th]. Judging from the solutions in this paper, it seems quite feasible that a Higgs-spectrum like that of Eq. (8), containing two $(!, 1,15)$ 's, without the $(1,1,6)$, and of course the desired matter sector, should be possible, but construc- 
tion of a single such solution is not yet in hand ( I thank Mirjam Cvetic for discussion in this respect).

[89] See e.g. D. Lewellen, Nucl. Phys. B 337, 61 (1990); A. Font, L. Ibanez and F. Quevedo, Nucl. Phys. B 345, 389 (1990); S. Chaudhuri, G. Hockney and J. Lykken, Nucl. Phys. B 456, 89 (1995) and hep-th/9510241; G. Aldazabal, A. Font, L. Ibanez and A. Uranga, Nucl. Phys. B 452, 3 (1995); ibid. B 465, 34 (1996); D. Finnell, Phys. Rev. D 53, 5781 (1996); A. A. Maslikov, I. Naumov and G. G. Volkov, Int. J. Mod. Phys. A 11, 1117 (1996); J. Erler, hep-th/9602032 and G. Cleaver, hep-th/9604183; and Z. Kakushadze and S. H. Tye, hep-th/9605221, and hep-th/9609027; Z. Kakushadze et al., hep-ph/9705202.

[90] P. Ginsparg, Phys. Lett. B 197, 139 (1987); V. S. Kaplunovsky, Nucl. Phys. B 307, 145 (1988); Erratum: ibid. B 382, 436 (1992).

[91] E. Witten, Nucl. Phys. B 471, 135 (1996); hep-th/9602070.

[92] see [91], and A.Hebecker, and M. Traplletti, Nucl. Phys. B 713, 173 (2005).

[93] J. C. Pati and K. S. Babu, "The Problems of Unification - Mismatch and Low $\alpha_{3}$ : A Solution with Light Vector-Like Matter", hep-ph/9606215, Phys. Lett. B 384, 140 (1996).

[94] J. C. Pati and P. Rastogi (unpublished); P. Rastogi, Ph.D. Thesis, Submitted to the University of Maryland (2006).

[95] I. Antoniadis, G. Leontaris and J. Rizos, Phys. Lett. B 245, 161 (1990); G. Leontaris, Phys. Lett. B 372, 212 (1996).

[96] A. E. Faraggi, Phys. Lett. B 278, 131 (1992); Phys. Lett. B 274, 47 (1992); Nucl. Phys. B 403, 101 (1993); A. E. Faraggi and E. Halyo, Nucl. Phys. B 416, 64 (1994); A. E. Faraggi, "Doublet-Triplet Splitting in Realistic Heterotic String Derived Mdels, Phys. Lett. B 520, 337 (2001), hep-ph/0107094.

[97] A. E. Faraggi and J. C. Pati, "A Family Universal Anomalous U(1) in String Models as the Origin of Supersymmetry Breaking and Squark-degeneracy", hep-ph/9712516v3, Nucl. Phys. B 256, 526 (1998).

[98] J. C. Pati, "The Essential Role of String Derived Symmetries in Ensuring Proton Stability and Light Neutrino Masses", hep-ph/9607446, Phys. Lett. B 388, 532 (1996).

[99] Y. Kawamura, Prog. Theor. Phys. 103, 613 (2000); G. Altarelli and F. Feruglio, Phys. Lett. B 511, 257 (2001); L. Hall and Y. Nomura, Phys. Rev. D 64, 055003 (2001); A. Hebecker and J. March-Russell, Nucl. Phys. B 613, 3 (2001); L. Hall and Y. Nomura, Phys. Rev. D 66, 075004 (2002); R. Barbieri, L. J. Hall and Y. Nomura, Nucl. Phys. B 624, 63 (2002); L. J. Hall, H. Murayama and Y. Nomura, Nucl. Phys. B 645, 85 (2002); Y. Nomura, Phys. Rev. D 65, 085036 (2002); A. Hebecker and J. March-Russell, Phys. Lett. B 539, 119 (2002); and M. L. Alciati et al., JHEP 0503, 054 (2005). 
[100] For orbifold SUSY SO(10) models in 6D, which use boundary conditions to break $S O(10)$ to an $U(1)_{\mathrm{X}^{-}}$extended-SM symmetry in $4 \mathrm{D}$ see : T. Asaka, W. Buchmuller and L. Covi, Phys. Lett. B. 523, 199 (2001); L. J. Hall, Y. Nomura, T. Okui and D. R. Smith, Phys. Rev. D 65, 035008 (2002). For orbifold SUSY SO(10) models in 5D, which combine boundary conditions with the Higgs mechanism on one of the branes to break $S O(10)$ to the $\mathrm{SM}$ in 4D, see e.g. R. Dermisek and A. Mafi, hep-ph/0108139; H.D. Kim and S. Raby, hep-ph/0212348; and B. Kyae and Q. Shafi, hep-ph/0312257. The last paper discusses inflation and leptogenesis in the context of such orbifold SUSY $\mathrm{SO}(10)$ models in 5D.

[101] L. J. Dixon, J. A. Harvey, C. Vafa and E. Witten, Nucl. Phys. B 261, 678 (1985); and Nucl. Phys. B 274, 285 (1986).

[102] D. J. Gross, J. A. Harvey, E. J. Martinec and R. Rohm, Phys. Rev. Lett. 54, 502 (1985).

[103] L. E. Ibanez, H. P. Nilles and F. Quevedo, Phys. Lett. B 187 (1987) 25; L. E. Ibanez, J. E. Kim, H. P. Nilles and F. Quevedo, Phys. Lett. B 191 (1987) 282.

[104] S. Dimopoulos and F. Wilczek, Proceedings Erice Summer School, ed. A. Zichichi (1981).

[105] K. S. Babu and S. M. Barr, Phys. Rev. D 50, 3529 (1994) hep-ph/9402291.

[106] V. Lucas and S. Raby, Phys. Rev. D 55, 6986 (1997) hep-ph/9610293. An interesting point noted here is that in certain range of SUSY parameters, especially with large $\tan \beta$, the LLRR operators for proton decay, often neglected, tend to dominate.

[107] R. Dermisek and S. Raby, Phys. Lett. B 622, 327 (2005) hep-ph/0507045; M. Albrecht, W. Altmannshofer, A. J. Buras, D. Guadagnoli and D. M. Straub, JHEP 0710, 055 (2007) arXiv:0707.3954 [hep-ph]].

[108] K. S. Babu, J. C. Pati and F. Wilczek (Ref. [25]).

[109] R. Dermisek, A. Mafi and S. Raby, Phys. Rev. D 63, 035001 (2001) hep-ph/0007213].

[110] K. S. Babu, J. C. Pati and Z. Tavartkiladze (Ref. [26]).

[111] J. C. Pati, "Neutrino Masses Shedding Light on Unification and Our Origin", talk presented at the Fujihara seminar, held at the KEK Laboratory, Tsukuba, Japan (Feb 23-25, 2004), arxiv: hep-ph/0407220; appeared in the proceedings; J. C. Pati, "A Unified Picture with Neutrino As a Central Feature", talk presented at the XI Int'l Workshop on "Neutrino Telescopes" held at Venice, Feb 21-25, 2005, arxiv: hepph/0507307, appeared in the Proceedings.

[112] K. S. Babu and R. N. Mohapatra, Phys. Rev. Lett. 70, 2845 (1993); M. C. Chen and K. T. Mahanthappa, Phys. Rev. D 62, 113007 (2000); B. Bajc, G. Senjanovic and F. Vissani, Phys. Rev. Lett. 90, 051802 (2003); H. S. Goh, R. N. Mohapatra and S. P. Ng, Phys. Lett. B 570, 215 (2003); C. S. Aulakh, B. Bajc, A. Melfo, G. Senjanovic 
and F. Vissani, Phys. Lett. B 588, 196 (2004); M. Bando, S. Kaneko, M. Obara and M. Tanimoto, Phys. Lett. B 580, 229 (2004); T. Fukuyama, A. Ilakovac, T. Kikuchi, S. Meljanac and N. Okada, Eur. Phys. J. C 42, 191 (2005); S. Bertolini, T. Schwetz and M. Malinsky, Phys. Rev. D 73, 115012 (2006); K. S. Babu and C. Macesanu, Phys. Rev. D 72, 115003 (2005); B. Dutta, Y. Mimura and R. N. Mohapatra, Phys. Rev. Lett. 100, 181801 (2008); C. S. Aulakh and S. K. Garg, Mod. Phys. Lett. A 24, 1711 (2009).

[113] C. H. Albright and S. M. Barr, Phys. Rev. D 64, 073010 (2001) [arXiv: hep$\mathrm{ph} / 0104294$.

[114] R. Dermisek and S. Raby, Phys. Lett. B 622, 327 (2005); R. Dermisek, M. Harada and S. Raby, Phys. Rev. D 74, 035011 (2006).

[115] See e.g. K. R. Dienes and J. March-Russell, hep-th/9604112; K. R. Dienes, hepth/9606467.

[116] These have been introduced in various forms in the literature. For a sample, see e.g., C. D. Frogatt and H. B. Nielsen, Nucl. Phys. B 147, 277 (1979); L. Hall and H. Murayama, Phys. Rev. Lett. 75, 3985 (1995); P. Binetruy, S. Lavignac and P. Ramond, Nucl. Phys. $B$ 477, 353 (1996). In the string theory context, see e.g., A. Faraggi, Phys. Lett. B 278, 131 (1992).

[117] If the effective non-renormalizable operator like $\mathbf{1 6}_{2} \mathbf{1 6} \mathbf{6}_{3} \mathbf{1 0} \mathbf{H}_{H} \mathbf{4 5}_{H} / M^{\prime}$ is induced through exchange of states with GUT-scale masses involving renormalizable couplings, rather than through quantum gravity, $M^{\prime}$ would, however, be of order GUT-scale. In this case $\left\langle\mathbf{4} \mathbf{5}_{H}\right\rangle / M^{\prime} \sim 1$, rather than $1 / 10$.

[118] While $\mathbf{1 6}_{H}$ has a GUT-scale VEV along the SM singlet, it turns out that it can also have a VEV of EW scale along the " $\tilde{\nu}_{L}$ " direction due to its mixing with $\mathbf{1 0}_{H}^{d}$, so that the $H_{d}$ of MSSM is a mixture of $\mathbf{1 0}_{H}^{d}$ and $\mathbf{1 6} \mathbf{H}_{H}^{d}$. This turns out to be the origin of non-trivial CKM mixings (See Ref. [25]).

[119] The flavor charge(s) of $\mathbf{4} \mathbf{5}_{H}\left(\mathbf{1 6}_{H}\right)$ would get determined depending upon whether $p(q)$ is one or zero (see below).

[120] The basic presumption here is that effective dimensionless couplings allowed by $S O(10) / G(224)$ and flavor symmetries are of order unity [i.e., $\left(h_{i j}, g_{i j}, a_{i j}\right) \approx 1 / 3-3$ (say)]. The need for appropriate powers of $(S / M)$ with $\langle S\rangle / M \sim M_{\mathrm{GUT}} / M_{\text {string }} \sim$ $(1 / 10-1 / 20)$ in the different couplings leads to a hierarchical structure. As an example, consider just one U(1)-flavor symmetry with one singlet $\mathrm{S}$. The hierarchical form of the Yukawa couplings exibited in Eqs. (7) and (8) would be allowed, for the case of $p=1, q=0$, if $\left(\mathbf{1 6}_{3}, \mathbf{1 6}_{2}, \mathbf{1 6}_{1}, \mathbf{1 0}_{H}, \mathbf{1 6}_{H}, \mathbf{4 5}_{H}\right.$ and $\left.\mathrm{S}\right)$ are assigned $\mathrm{U}(1)$-charges of ( $a$, $a+1, a+2,-2 a,-a-1 / 2,0,-1)$. It is assumed that other fields are present that would make the U(1) symmetry anomaly-free. The value of "a" would get fixed by the presence of other operators (see later). 
[121] These effective non-renormalizable couplings can of course arise through exchange of (for example) $\mathbf{4 5}$ in the string tower, involving renormalizable $\mathbf{1 6}_{i} \overline{\mathbf{1 6}}_{H} \mathbf{4 5}$ couplings. In this case, one would expect $M \sim M_{\text {string }}$.

[122] K. S. Babu, J. C. Pati and P. Rastogi, "Tying in CP and flavor violations with fermion masses and neutrino oscillations", hep-ph/04010200, Phys. Rev. D 71, 015005 (2005).

[123] The expressions $V_{\mathrm{cb}}$ and $\theta_{23}^{\nu}$ are given in Eq. (12) in terms of the simple square-rootformulas only to exhibit the qualitative reason based on symmetry (explained in the text) for the enhancement of $\theta_{23}^{\nu}$ and suppression of $V_{\mathrm{cb}}$. The simple formulas do not really hold, however, when each square-root-term is rather large, as in $\theta_{23}^{\nu}$. The values quoted in Eq. (12) are based on exact numerical calculations. 25]

[124] It has been noted[111] that small intrinsic (non-seesaw) masses $\sim($ few $) \times 10^{-3} \mathrm{eV}$ of the LH neutrinos can arise quite plausibly through higher dimensional operators of the form: $W_{12} \supset \kappa_{12} \mathbf{1 6}_{1} \mathbf{1 6}_{2} \mathbf{1 6}_{H} \mathbf{1 6}_{H} \mathbf{1 0}_{H} \mathbf{1 0}_{H} / M_{\text {eff }}^{3}$, without involving the standard seesaw mechanism, and in accord with the $\mathrm{U}(1)$ flavor symmetry if a $=1 / 2$ (see Ref. [120]). One can verify that such a term would lead to an intrinsic Majorana mixing mass term of the form $m_{12}^{0} \nu_{L}^{e} \nu_{L}^{\mu}$, with a strength given by $m_{12}^{0} \approx \kappa_{12}\left\langle\mathbf{1 6}_{H}\right\rangle^{2}(175 \mathrm{GeV})^{2} / M_{\text {eff }}^{3} \sim$ $(1.5-6) \times 10^{-3} \mathrm{eV}$, for $\left\langle\mathbf{1 6}_{H}\right\rangle \approx(1-2) M_{\mathrm{GUT}}$ and $\kappa_{12} \sim 1$, if $M_{\mathrm{eff}} \sim M_{\mathrm{GUT}} \approx 2 \times 10^{16}$ $\mathrm{GeV}[111]$. Such an intrinsic Majorana $\nu_{e} \nu_{\mu}$ mixing mass $\sim$ few $\times 10^{-3} \mathrm{eV}$, though small compared to $m\left(\nu_{3}\right)$, is still much larger than what one would generically get for the corresponding term from the standard seesaw mechanism. Now, the diagonal $\left(\nu_{L}^{\mu} \nu_{L}^{\mu}\right)$ mass-term, arising from the standard seesaw mechanism is expected to be $\sim(3-10) \times$ $10^{-3} \mathrm{eV}$ for a natural value of $|y| \approx 1 / 20-1 / 15$, say[25]. Thus, taking the net values of $m_{22}^{0} \approx(8-10) \times 10^{-3} \mathrm{eV}, m_{12}^{0} \approx 3 \times 10^{-3} \mathrm{eV}$ as above and $m_{11}^{0} \ll 10^{-3} \mathrm{eV}$, which are all plausible, we obtain $m_{\nu_{2}} \approx(7-9) \times 10^{-3} \mathrm{eV}, m_{\nu_{1}} \sim(1$ to few $) \times 10^{-3} \mathrm{eV}$, so that $\Delta m_{21}^{2} \approx(6-8) \times 10^{-5} \mathrm{eV}^{2}$ and $\theta_{12}^{\nu} \approx\left(20^{\circ}-35^{\circ}\right)$, in accord with the data, which show $\theta_{12}^{\nu} \approx 33^{\circ}$. (For review and notations see Refs. [125] and [126]). Likewise, a non-seesaw contribution can be used to plausibly obtain $\theta_{13}^{\nu} \approx\left(2^{\circ}-10^{\circ}\right)$ to be compared with the observed value of $\approx 9^{\circ}$. (See Refs. [125] and [126]).

[125] Particle Data Group Review: "Neutrino Mass, Mixing and Oscillations" by K. Nakamura and S. T. Petcov, Pdg.lbl.gov/2016/reviews/rpp2016-rev-neutrinomixing.pdf, Oct. 2016. Best fit values quoted here are: $\sin ^{2} \theta_{23}=0.437$ or 0.569 .

[126] For a recent review of experimental and theoretical aspects of neutrino physics, the latter being in the context of unification models possessing flavor symmetries, see S.F. King, arXiv: 1701.04413, and references therein. The global fits quoted here, for the case of normal hierarchy, yield (for one sigma range): $\theta_{23} \approx 41.4^{\circ} \pm 1.6^{\circ}, \theta_{12} \approx$ $33.2^{\circ} \pm 1.2^{\circ}, \theta_{13} \approx 8.45^{\circ} \pm 0.15^{\circ}$. The best fit (for normal ordering) yields: $\Delta m_{31}^{2}=$ $(2.55 \pm 0.05) \times 10^{-3} \mathrm{eV}^{2} ; \Delta m_{21}^{2}=(7.45 \pm 0.25) \times 10^{-5} \mathrm{eV}^{2}$.

[127] P.A.R. Ade et al. [PLANCK collaboration], Astron. Astrophs. 594, A13 (2016).

[128] K. S. Babu J. C. Pati and P. Rastogi," Lepton Flavor Violation within a realistic SO(10)/G(2,2,4) Framework", hep-ph/0502152, Phys. Lett. B. (2005); P. Rastogi, Ph.D. Thesis, Univ. of Maryland (2006). 
[129] S. M. Barr, Phys. Lett. B 112, 219 (1982); J. P. Derendinger, J. E. Kim and D. V. Nanopoulos, Phys. Lett. B 139, 170 (1984); I. Antoniadis, J. Ellis, J. Hagelin and D. V. Nanopoulos, Phys. Lett. B 194, 231 (1987).

[130] For reviews on different aspects of leptogenesis, see e.g. W. Buchmuller, R. D. Peccei and T. Yanagida, Ann Rev. Nucl. Part. Sci. 55, 311 (2005); S. Davidson, E. Nardi and Y. Nir, Phys. Rept. 466, 105 (2008); S. Blanchet and P. Di Bari, New J. Phys. 14, 125012 (2012); C.S. Fong, E. Nardi and A. Riotto, Adv. High Energy Phys. 2012, 158303 (2012).

[131] J. C. Pati, "Leptogenesis within a Predictive $G(2,2,4) / S O(10)$ Framework", Phys. Rev. D 68, 072002 (2003).

[132] W. Buchmuller, P. Di Bari and M. Plumacher, hep-ph/0401240; G. F. Giudice, A. Notart, M. Raidal, A. Riotto and A. Strumia, hep-ph/0310123.

[133] WMAP Collaboration, D. N. Spergel et al., Astrophys. J. Suppl 148, 175 (2003); astro-ph/0310723, yielded: $\left(Y_{B}\right)_{\mathrm{WMAP}} \approx(8.7 \pm 0.4) \times 10^{-11}$. The latest results based on measurements of CMB anisotropies by the PLANCK Collaboration (quoted in the Particle Data Group Review (2016)) lead to: $Y_{B} \approx(8.65 \pm 0.10) \times 10^{-11}$.

[134] S. Weinberg, Phys. Rev. Lett. 48, 1303 (1982).

[135] M. Kawasaki, K. Kohri, T. Moroi and A. Yotsuyanagi, Phys. Rev. D 78, 065011, 2008; M. Kawasaki, K. Kohri and T. Moroi, astro-ph/0402990.

[136] See, e.g., T.Moroi, H. Murayama and M. Yamaguchi, Phys.Lett. B 303, 289 (1993) and references there in.

[137] See R. Jeannerot, S. Khalil, G. Lazarides and Q. Shafi, " Inflation and monopoles in supersymmetric $S U(4)^{c} \times S U(2)(L) \times S U(2)(R)$ ", J. High Energy Physics $012(2000)$; hep-ph/0002151 and references there in. For a review on supersymmetric hybrid inflation, see e.g. G. Lazarides, arXiv: hep-ph/0607032. For a recent work on inflationary non-thermal leptogenesis based on a supersymmetric $G(2,2,4)$ model, see C. Pallis and N. Toumbas, arXiv: hep-ph/1207.3730.

[138] B. Charles Bryant and S. Raby "A Pati-Salam Version of Subcritical Hybrid Inflation", arXiv: 1601. 03749; B. Charles Bryant, Z. Poh, " Reheating and Leptogenesis after Pati-Salam F-term Subcritical Hybrid Inflation", arXiv:1612.04382.

[139] G. Lazarides, Iain N. R. Peddle, and A. Vamvasakis, arXiv:hep-ph/0804.3661.

[140] Incorporating such an effective super potential in accord with the assignment of flavor charges suggested in Refs. [120] and [124] (for this purpose, $\Phi$ and $\Phi$ are quivalent to $\overline{16}_{\mathrm{H}}$ and $16_{\mathrm{H}}$ respectively) would involve two additional signlets with appropriate flavor charges. One or both of those may have a VEV $\sim \mathrm{M}$.

[141] J. Hisano, H. Murayama and T. Yanagida, Nucl. Phys. B 402, 406 (1993) hepph/9207279]; J. Hisano, arXiv: hep-ph/0107266. 
[142] N. Sakai and T. Yanagida, Nucl. Phys. B 197, 533 (1982); S. Weinberg, Phys. Rev. D 26, 287 (1982); S. Dimopoulos, S. Raby and F. Wilczek, Phys. Lett. B, 112133 (1982).

[143] H. Murayama and A. Pierce, Phys. Rev. D 65, 055009 (2002) hep-ph/0108104.

[144] B. Bajc, P. Fileviez Perez and G. Senjanovic, arXiv:hep-ph/0210374; I. Gogoladze and A. Kobakhidze, Phys. Atom. Nucl. 60, 126 (1997) [Yad. Fiz. 60N1, 136 (1997)] hep-ph/9610389.

[145] J. C. Pati, "Confronting The Conventional ideas on Grand Unification With Fermion Masses, Neutrino Oscillations and Proton Decay", hep-ph/0204240; Proceedings of the ICTP, Trieste School 2001 and Proceedings of the DAE Meeting, Allahabad, India (2002).

[146] Note that in the presence of a second $\mathrm{SO}(10)$-singlet field $\mathbf{S}^{\prime}$ carrying flavor charge of $2 \mathrm{a}+1 / 2$, an effective coupling of the form $\mathbf{1 6}_{\mathbf{H}} \cdot \overline{\mathbf{1 6}_{\mathbf{H}}} \cdot \mathbf{S}^{\prime}$ would be allowed preserving the $\mathrm{U}(1)$-flavor symmetry introduced in Sec. 4. With $\mathbf{S}^{\prime}$ having a VEV of GUT-scale, such a coupling would generate a mass-term $\mathbf{1 6}_{\mathbf{H}} \cdot \overline{\mathbf{1 6}_{\mathbf{H}}}$ that enters into Fig. 4 . The presence of this second singlet $\mathbf{S}^{\prime}$ does not in any way affect the hierarchical pattern of effective couplings exhibited in Eqs. (7) and (9). I thank Qaisar Shafi for raising this point.

[147] Thus the new set of proton decay operators become proportional to $\left(f_{i j}\right)_{\nu} g_{k l} K\left\langle\overline{\mathbf{1 6}}_{H}\right\rangle\left\langle\mathbf{1 6}_{H}\right\rangle /\left(M^{2}\right)\left(M_{16}\right)$ where $M \approx M_{\mathrm{st}}$ and $M_{16}\left(\sim M_{\mathrm{GUT}}\right)$ is the mass of the $\mathbf{1 6}_{H}$ (see Ref. [145] for a discussion limiting the strength of this operator).

[148] The CMS collaboration, CMS-PAS-SUS-16-024; CMS-PAS-SUS-16-028; The ATLAS collaboration, ATLAS-CONF-2015-067;ATLAS-CONF-2016-050.

[149] Very recently, additional restrictions on the mass of the light stop depending upon the mass of the lighter neutralino, including some cases of near degeneracy in their masses, have been reported on behalf of the ATLAS and CMS collaborations by A. Petridis at the Moriond Meeting, La Thuille, Italy, March 18-25, 2017. For some details of the CMS Analysis, see CMS PAS SUS-16-049. Certain range in the choice of SUSY parameters for the case of inverted hierarchy, including variants, that we have followed for consideration of proton lifetimes, being guided by the choice in Ref. [150], seems to be quite compatible with the improved searches for light stop as above. This is so especially with the latter having a mass above about $700 \mathrm{GeV}$ and the neutralino being within about ( 50 - 100 ) GeV below it. Such variations in the SUSY spectrum will not alter our predictions for upper limits on proton lifetimes by more than 10 percent.

[150] M. Badziak, E. Dudas, M. Olechowski and S. Pokorski, "Inverted sfermion mass hierarchy and the Higgs boson mass in the MSSM" , JHEP 07, 155 (2012); arXiv: 1205.1675; see references therein for inverted hierarchy.

[151] For a theoretical analysis of the pre-Moriond LHC data [148], see e.g. C. Han, J. Ren, L. Wu, J. M. Yang and M. Zhang, arXiv: 1609.02361; C. Han, M. Nojiri, M. Takeuchi, T. Yanagida, arXiv: 1609.09303 and references there in. 
[152] For constraints on light stop $(\sim 500 \mathrm{GeV})$ and discussion of radiative SUSY naturalness, see a recent paper by H. Baer, V. Barger, N. Nagata and M. Savoy, arXiv: 1611.08511.

[153] T. Friedmann and E. Witten, Adv. Th.Math.Phys 7577 (2003); hep-th/0211269.

[154] S. M. Barr and S. Raby, Phys. Rev. Lett 79, 4748 (1997); hep-ph/9705366.

[155] J. Feng," Naturalness and the Status of Supersymmetry" Ann.Rev.Nucl.Sci. 63 (2013); arXiv:1302.6587.

[156] H. Baer, V. Barger, P. Huang and X. Tata, JHEP 1205, 109 (2012).

[157] J. C. Pati, A. Salam and U. Sarkar, Phys. Lett. B 133, 330 (1983); K. S. Babu and R. N. Mohapatra, Phys. Rev. Lett. 100, 091803 (2012). 